\title{
Parabolic Whittaker functions and topological field theories I
}

\author{
Anton Gerasimov, Dimitri Lebedev and Sergey Oblezin
}

First, we define a generalization of the standard quantum Toda chain inspired by a construction of quantum cohomology of partial flags spaces $G L_{\ell+1} / P, P$ a parabolic subgroup. Common eigenfunctions of the parabolic quantum Toda chains are generalized Whittaker functions given by matrix elements of infinite-dimensional representations of $\mathfrak{g l}_{\ell+1}$. For maximal parabolic subgroups (i.e., for $P$ such that $G L_{\ell+1} / P=\mathbb{P}^{\ell}$ ) we construct two different representations of the corresponding parabolic Whittaker functions as correlation functions in topological quantum field theories on a two-dimensional disk. In one case the parabolic Whittaker function is given by a correlation function in a type- $A$ equivariant topological sigma model with the target space $\mathbb{P}^{\ell}$. In the other case, the same Whittaker function appears as a correlation function in a type- $B$ equivariant topological Landau-Ginzburg model related with the type- $A$ model by mirror symmetry. This note is a continuation of our project of establishing a relation between twodimensional topological field theories (and more generally topological string theories) and Archimedean ( $\infty$-adic) geometry. From this perspective the existence of two, mirror dual, topological field theory representations of the parabolic Whittaker functions provide a quantum field theory realization of the local Archimedean Langlands duality for Whittaker functions. The established relation between the Archimedean Langlands duality and mirror symmetry in two-dimensional topological quantum field theories should be considered as a main result of this note.

\section{Introduction}

In $[17,18]$ we propose two-dimensional topological field theories as a proper framework for a description of the Archimedean completion of arithmetic schemes ( $\infty$-adic geometry according to [28]). In particular, we give a representation of local Archimedean $L$-factors (we include local epsilon-factor in the definition of the $L$-factors) in terms of two-dimensional topological field theories. It is well-known that local $L$-factors allow two types of 
constructions - "arithmetic" construction based on representation theory of the Weil-Deligne group of the local field and "automorphic" construction relying on representation theory of reductive groups over local field (see, e.g., $[1,6,7])$. The equivalence of these constructions for various types of $L$-factors is a subject of the local Langlands duality. In an interpretation suggested in $[17,18]$ the "arithmetic" construction of local Archimedean $L$-factors is naturally identified with a type- $A$ topological field theory description [17] in terms of equivariant volumes of spaces of holomorphic maps of a disk into complex vector spaces. The "automorphic" construction of the same local $L$-factors is realized using a type- $B$ topological field theory via periods of holomorphic forms [18]. The Archimedean Langlands duality between these two constructions of the local Archimedean $L$-factors appears as a mirror duality between underlying type- $A$ and type- $B$ topological sigma models.

The duality between two constructions of $L$-factors can be extended to a duality between two constructions of the Whittaker functions. In the nonArchimedean case, this duality manifests in the existence of the ShintaniCasselman-Shalika (SCS) formula $[8,31]$ for the non-Archimedean Whittaker functions along with a standard realization of the Whittaker functions as matrix elements of representations of reductive groups $G$ over local nonArchimedean fields. According to $[8,31]$ the non-Archimedean Whittaker functions can be identified with characters of finite-dimensional representations of Langlands dual groups ${ }^{\mathrm{L}} G$. In [16] we propose a $q$-version of the classical SCS formula providing a $q$-version of the Langlands duality pattern for the $q$-deformed Whittaker functions. In appropriate limit the $q$-version of SCS formula reduces to the non-Archimedean one. The limiting case provides an Archimedean analog of the results of $[8,31]$. This leads to an explicit realization of the Langlands duality pattern for the Archimedean Whittaker functions.

In this note, the approach of $[17,18]$ to a construction of local Archimedean $L$-factors in terms of topological field theories is generalized to a class of Whittaker functions introduced below. Recall that standard $\mathfrak{g l}_{\ell+1^{-}}$-Whittaker functions are common eigenfunctions of quantum $\mathfrak{g l}_{\ell+1^{-}}$ Toda chain Hamiltonians and according to [21] appear in a description of $S^{1} \times U_{\ell+1}$-equivariant Gromov-Witten invariants of complete flag spaces $G L_{\ell+1}(\mathbb{C}) / B, B$ a Borel subgroup. In the first part of this note, we introduce a class of generalized $\mathfrak{g l}_{\ell+m}$-Whittaker functions associated with a parabolic subgroup $P \subset G L_{\ell+m}(\mathbb{C})$. We conjecture that a $P$-parabolic $\mathfrak{g l}_{\ell+m}$-Whittaker function describes $S^{1} \times U_{\ell+m}$-equivariant Gromov-Witten invariants of the partial flag space $G L_{\ell+m}(\mathbb{C}) / P$. The parabolic $\mathfrak{g l}_{\ell+m}$-Whittaker functions are common eigenfunctions of quantum Hamiltonians of generalized quantum Toda chain. We explicitly check, in the case of 
$G L_{\ell+m}(\mathbb{C}) / P=G r(m, \ell+m)$, that the corresponding Toda chain recovers Astashkevich-Sadov-Kim description $[2,27]$ of quantum cohomology of Grassmannian $\operatorname{Gr}(m, \ell+m)$. In the case $m=1$, we explicitly verify that the corresponding parabolic Whittaker functions describe equivariant quantum cohomology of $G r(1, \ell+1)=\mathbb{P}^{\ell}$.

In the second part of this note, we propose two representations of the parabolic Whittaker functions associated with maximal parabolic subgroup $P$ (i.e., such that $G L_{\ell+1}(\mathbb{C}) / P=\mathbb{P}^{\ell}$ ) as correlation functions in two-dimensional topological fields theories on a disk. The first representation (see Theorem 2.1) is given in terms of an equivariant type- $A$ topological sigma model with a target space $\mathbb{P}^{\ell}$ and provides an integral representation of the parabolic Whittaker function of the Mellin-Barnes type. In this representation, the Whittaker function arises as an equivariant volume of a space of holomorphic maps of the disk into projective space $\mathbb{P}^{\ell}$ and thus, following [17], shall be considered as an "arithmetic" construction of the parabolic Whittaker function. This representation provides an Archimedean analog of SCS formula $[8,31]$ for the parabolic Whittaker functions. The second representation is given in terms of a type- $B$ topological Landau-Ginzburg model on a disk with a target space $\mathbb{C}^{\ell+2}$ supplied with appropriate superpotential (see Theorem 3.1). In this description, the parabolic Whittaker function is given by a holomorphic period [19] (see also [4, 9, 20]). In contrast with the type- $A$ model representation the correlation functions in $B$ model can be reduced to certain finite-dimensional integrals with simple integrands. Thus obtained finite-dimensional integral representation naturally arises in a matrix element interpretation of the Whittaker function according to [14]. Hence, in analogy with [18], the type- $B$ topological field theory representation shall be considered as an "automorphic"/representation theory construction of the parabolic Whittaker functions. The discussed type- $A$ and type- $B$ topological quantum field theories are related by mirror transformation. This leads to interpretation of the Archimedean Langlands duality between "arithmetic" and "automorphic" constructions of the parabolic Whittaker functions as a mirror duality between underlying topological field theories.

Let us remark that pairs of Langlands dual Lie groups already appear in various instances of mirror symmetry (see, e.g. [25]). In particular construction of a mirror dual description of type- $A$ topological sigma models associated with flag spaces $G / B$ in terms of eigenfunctions of the quantum Toda chains associated with the dual Lie groups $G^{\vee}[21,22]$. In this paper we establish a relation of these results with the local arithmetic (Archimedean) Langlands duality. Note that the global geometric Langlands correspondence due to [5] allows an interpretation in terms of $S$-duality in four-dimensional 
topological Yang-Mills theories, which can be reformulated as a mirror symmetry of the associated moduli spaces [26]. It would be interesting to understand a possible relation between local arithmetic and geometric Langlands dualities using quantum field theory interpretation.

Finally, let us note that the results of this note can be generalized to the case of general parabolic Whittaker functions. For a general parabolic subgroup, the corresponding parabolic version of the quantum $\mathfrak{g l}_{\ell+m}$-Toda chain provides a new interesting example of a quantum integrable system and will be considered elsewhere. One should also stress that explicit calculations of correlation functions in topological field theories on non-compact manifolds is an interesting subject by itself and undoubtedly deserves further attention. According to the standard lore boundary conditions in topological field theories encode geometry of topological branes in a target space. It would be interesting to compare the choice of the boundary conditions used in this note with an equivariant generalization of the standard brane boundary conditions. We are going to clarify this relation elsewhere.

The plan of the paper is as follows. In Section 1, we introduce parabolic generalizations of the standard $\mathfrak{g l}_{\ell+m}$-Whittaker function as particular matrix elements of infinite-dimensional representations of Lie algebra $\mathfrak{g l}_{\ell+m}$. In Section 2, we construct a representation of the parabolic $\mathfrak{g l}_{\ell+1}$-Whittaker functions associated with a maximal parabolic subgroup as correlation functions of a type- $A$ topological sigma model on a disk with a target space $\mathbb{P}^{\ell}$. In Section 3, we provide a mirror dual representation of the same Whittaker function as a correlation function in a type- $B$ topological Landau-Ginzburg model on a disk. In Section 4, we give a heuristic derivation of the result of Sections 2 and 3. In Section 5, we discuss directions of further research. Finally, in Appendixes the proofs and some technical results are given.

\section{Parabolic Whittaker functions}

In this section, we introduce a generalization of $\mathfrak{g l}_{\ell+m}$-Whittaker functions associated with a parabolic subgroup $P \subset G L_{\ell+m}(\mathbb{C})$. The standard $\mathfrak{g l}_{\ell+m^{-}}$ Whittaker functions are associated with Borel subgroups $B \subset G L_{\ell+m}(\mathbb{C})$ and are common eigenvalues of quantum $\mathfrak{g l}_{\ell+m}$-Toda chain Hamiltonians (for standard facts on quantum Toda chains see, e.g., [30]). The classical Whittaker functions are relevant to a description of (equivariant) Gromov-Witten invariants of flag spaces $G / B$ [21]. The parabolic generalizations of Whittaker functions introduced below are common eigenvalues of generalized quantum Toda chains defined below. In the next sections, we demonstrate that the parabolic Whittaker functions describe equivariant Gromov-Witten invariants of partial flag spaces. In this section, we restrict considerations to 
the case of the parabolic subgroup $P_{m, \ell+m}$ such that $G L_{\ell+m}(\mathbb{C}) / P_{m, \ell+m}=$ $G r(m, \ell+m)$ and refer to the corresponding Whittaker functions as $(m, \ell+$ $m$ )-Whittaker functions. The general case follows basically the same pattern and will be treated elsewhere.

First, we recall the representation theory construction of the standard $\mathfrak{g l}_{\ell+1}$-Whittaker functions. Let $E_{i j}, i, j=1, \ldots, \ell+1$ be the standard basis of the Lie algebra $\mathfrak{g l}_{\ell+1}$. Let $\mathcal{Z}\left(\mathcal{U} \mathfrak{g l}_{\ell+1}\right) \subset \mathcal{U} \mathfrak{g l}_{\ell+1}$ be the center of the universal enveloping algebra $\mathcal{U} \mathfrak{g l}_{\ell+1}$. Let $B_{ \pm} \subset G L_{\ell+1}(\mathbb{C})$ be upper-triangular and lower-triangular Borel subgroups and $N_{ \pm} \subset B_{ \pm}$be upper-triangular and lower-triangular unipotent subgroups. We denote $\mathfrak{b}_{ \pm}=\operatorname{Lie}\left(B_{ \pm}\right)$and $\mathfrak{n}_{ \pm}=\operatorname{Lie}\left(N_{ \pm}\right)$their Lie algebras. Let $\mathfrak{h} \subset \mathfrak{g l}_{\ell+1}$ be a diagonal Cartan subalgebra and $S_{\ell+1}$ be the associated Weyl group of $G L_{\ell+1}$. Using the HarishChandra isomorphism of $\mathcal{Z}\left(\mathcal{U}_{\mathfrak{g l}} \mathfrak{l}_{\ell+1}\right)$ with the Weyl group invariant subalgebra of the symmetric algebra of the Cartan subalgebra $\mathfrak{h}$ we identify central characters with homomorphisms $c: \mathbb{C}\left[h_{1}, \ldots, h_{\ell+1}\right]^{S_{\ell+1}} \rightarrow \mathbb{C}$ of an algebra of $S_{\ell+1}$-invariant polynomials of the generators of $\mathfrak{h}$ into complex numbers. Let $\pi_{\underline{\lambda}}: \mathcal{U}_{\mathfrak{g l}_{\ell+1}} \rightarrow \operatorname{End}\left(\mathcal{V}_{\underline{\lambda}}\right), \mathcal{V}_{\underline{\lambda}}=\operatorname{Ind}_{\mathcal{U}^{\prime} \mathfrak{b}_{-}}$be a family of principal series

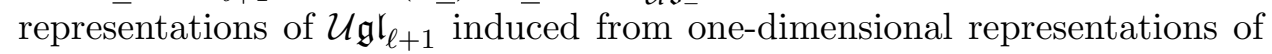
$\mathcal{U} \mathfrak{b}_{-}$such that images of the symmetric polynomials of $h_{i}$ are symmetric polynomials of $\lambda_{j} \in \mathbb{C}, \underline{\lambda}=\left(\lambda_{1}, \ldots, \lambda_{\ell+1}\right) \in \mathbb{C}^{\ell+1}$. Let $\mathcal{V}_{\lambda}^{\prime}$ be a dual module supplied with induced action of $\mathcal{U} \mathfrak{g l}_{\ell+1}^{\text {opp }}$ (universal enveloping algebra of $\mathfrak{g l}_{\ell+1}^{\text {opp }}$ obtained by inverting the signs of the structure constants of $\mathfrak{g l}_{\ell+1}$ ). Denote $\langle$,$\rangle the pairing between \mathcal{V}_{\underline{\lambda}}^{\prime}$ and $\mathcal{V}_{\underline{\lambda}}$. We suppose that the action of the Cartan subalgebra $\mathfrak{h}$ in representation $\mathcal{V}_{\underline{\lambda}}$ can be integrated to the action of the corresponding Cartan subgroup $H \subset G L_{\ell+1}(\mathbb{C})$.

According to Kostant, the $\mathfrak{g l}_{\ell+1}$-Whittaker function can be defined as a matrix element

$$
\Psi_{\underline{\lambda}}\left(x_{1}, \ldots, x_{\ell+1}\right)=\mathrm{e}^{-\rho(x)}\left\langle\psi_{\mathrm{L}}\left|\pi_{\underline{\lambda}}\left(\mathrm{e}^{-\sum_{i=1} x_{i} E_{i i}}\right)\right| \psi_{\mathrm{R}}\right\rangle
$$

where the vectors $\left\langle\psi_{\mathrm{L}}\right| \in \mathcal{V}_{\underline{\lambda}}^{\prime}$ and $\left|\psi_{\mathrm{R}}\right\rangle \in \mathcal{V}_{\underline{\lambda}}$ provide one-dimensional representations of $N_{-}$and $N_{+}$correspondingly

$$
\left\langle\psi_{\mathrm{L}}\left|E_{i+1, i}=-\left\langle\psi_{\mathrm{L}}\left|, \quad E_{i, i+1}\right| \psi_{\mathrm{R}}\right\rangle=\right| \psi_{\mathrm{R}}\right\rangle, \quad i=1, \ldots, \ell
$$

and $\rho_{r}=(1 / 2)(\ell+2-2 k), k=1, \ldots, \ell+1$ are the components of the vector $\rho$ in $\mathbb{R}^{\ell+1}$. The standard considerations (see, e.g., [30]) show that matrix element (1.1) is a common eigenfunction of a family of commuting differential operators descending from the action of the generators of $\mathcal{Z}\left(\mathcal{U}_{\mathfrak{g l}_{\ell+1}}\right)$ in $\mathcal{V}_{\underline{\lambda}}$. These differential operators can be identified with quantum Hamiltonians of $\mathfrak{g l}_{\ell+1}$-Toda chain. 
Below we propose a generalization of the $\mathfrak{g l}_{\ell+m}$-Whittaker functions (1.1) (for convenience we slightly change notations replacing $\ell+1$ by $\ell+m$ ). Let $P_{m, \ell+m}$ be a parabolic subgroup of $G L_{\ell+m}(\mathbb{C})$ such that the corresponding Levi factor is $G L_{m}(\mathbb{C}) \times G L_{\ell}(\mathbb{C})$. The corresponding partial flag space $G L_{\ell+m}(\mathbb{C}) / P_{m, \ell+m}$ is isomorphic to Grassmannian $G r(m, \ell+m)$. The associated Whittaker function is then defined as the following matrix element of the principle series representation $\mathcal{V}_{\underline{\lambda}}=\operatorname{Ind}_{\mathcal{U}\left(\mathfrak{b}_{-}\right)}^{\mathcal{U}\left(\mathfrak{l}_{\ell+m}\right)}$. Let us associate with $P_{m, \ell+m}$ a decomposition of the Borel subalgebra $\mathfrak{b}_{+} \subset \mathfrak{g l}_{\ell+m}$

$$
\mathfrak{b}_{+}=\mathfrak{h}^{(m, \ell+m)} \oplus \mathfrak{n}_{+}^{(m, \ell+m)},
$$

with the commutative subalgebra $\mathfrak{h}^{(m, \ell+m)} \subset \mathfrak{b}_{+}$generated by

$$
\begin{array}{ll}
H_{1}=E_{11}+\cdots+E_{m m}, & H_{k}=E_{1, k}, \quad 2 \leq k \leq m, \\
H_{m+k}=E_{m+k, \ell+m}, \quad 1 \leq k \leq \ell-1, & H_{\ell+m}=E_{m+1, m+1}+\cdots+E_{\ell+m, \ell+m},
\end{array}
$$

and the subalgebra $\mathfrak{n}_{+}^{(m, \ell+m)} \subset \mathfrak{b}_{+}$given by

$$
\begin{gathered}
\mathfrak{n}_{+}^{(m, \ell+m)}=\left\langle E_{1, \ell+m} ; E_{1, m+1} ; E_{m, \ell+m} ; E_{k k}, 2 \leq k \leq \ell+m-1 ;\right. \\
\left.E_{j, j+1}, 2 \leq j \leq \ell+m-2\right\rangle .
\end{gathered}
$$

Note that $\operatorname{dim} \mathfrak{h}^{(m, \ell+m)}=\operatorname{rank} \mathfrak{g l}_{\ell+m}=\ell+m$ and $\operatorname{dim} \mathfrak{n}_{+}^{(m, \ell+m)}=(\ell+$ $m)(\ell+m-1) / 2$. Let $H^{(m, \ell+m)}$ and $N_{+}^{(m, \ell+m)}$ be the Lie groups corresponding to the Lie algebras $\mathfrak{h}^{(m, \ell+m)}$ and $\mathfrak{n}_{+}^{(m, \ell+m)}$. An open part $\dot{G} L_{\ell+m}$ of $G L_{\ell+m}$ allows the following analog of the Gauss decomposition:

$$
\dot{G} L_{\ell+m}=N_{-} H^{(m, \ell+m)} N_{+}^{(m, \ell+m)} .
$$

Definition 1.1. The Whittaker vectors $\left\langle\psi_{\mathrm{L}}\left|\in \mathcal{V}_{\underline{\lambda}}^{\prime},\right| \psi_{\mathrm{R}}\right\rangle \in \mathcal{V}_{\underline{\lambda}}$ are defined by the following conditions:

$$
\begin{aligned}
& \left\langle\psi_{\mathrm{L}}\right| E_{n+1, n}=\hbar^{-1}\left\langle\psi_{\mathrm{L}}\right|, \quad 1 \leq n \leq \ell+m-1, \\
& \begin{cases}E_{k k}\left|\psi_{\mathrm{R}}\right\rangle=0, & 2 \leq k \leq \ell+m-1, \\
E_{k, k+1}\left|\psi_{\mathrm{R}}\right\rangle=0, & 2 \leq k \leq \ell+m-2, \\
E_{1, m+1}\left|\psi_{\mathrm{R}}\right\rangle=0, & l \neq 1, \\
E_{m, \ell+m}\left|\psi_{\mathrm{R}}\right\rangle=0, & m \neq 1, \\
E_{1, \ell+m}\left|\psi_{\mathrm{R}}\right\rangle=(-1)^{\epsilon(\ell, m)} \frac{1}{\hbar}\left|\psi_{\mathrm{R}}\right\rangle, & \end{cases}
\end{aligned}
$$

where $\epsilon(\ell, m)$ is an integer number and $\hbar \in \mathbb{R}$. 
Here, in comparison with (1.2), we introduce additional parameter $\hbar$ to make a contact with the results of other Sections $((1.2)$ corresponds to $\hbar=1)$. Note that Equations (1.6) define a one-dimensional representation $\left\langle\psi_{\mathrm{L}}\right|$ of the Lie algebra $\mathfrak{n}_{-}$of strictly lower-triangular matrices and Equations (1.7) define a one-dimensional representation of $\mathfrak{n}_{+}^{(m, \ell+m)}$.

Definition 1.2. The $(m, \ell+m)$-Whittaker function associated with the principal series representation $\left(\pi_{\underline{\lambda}}, \mathcal{V}_{\underline{\lambda}}\right)$ is defined as the following matrix element:

$$
\Psi_{\underline{\lambda}}^{(m, \ell+m)}(x)=\mathrm{e}^{-\rho_{1}\left(x_{1}-x_{\ell+m}\right)}\left\langle\psi_{\mathrm{L}}\left|\pi_{\underline{\lambda}}(g(x))\right| \psi_{\mathrm{R}}\right\rangle
$$

where the left and right vectors solve Equations (1.6) and (1.7) respectively and $\rho_{1}=(\ell+m-1) / 2$. Here $g(x)$ is a Cartan group valued function given by

$$
g(x)=\exp \left\{-\sum_{i=1}^{\ell+m} x_{i} H_{i}\right\}
$$

where the generators $H_{i}, i=1, \ldots,(\ell+m)$ are defined by $(1.3)$.

Similar to the classical Whittaker functions $(m, \ell+m)$-parabolic Whittaker functions are naturally common eigenfunctions of a family of commuting differential operators. Let us define a set of mutually commuting differential operators $\mathcal{H}_{k}^{(m, \ell+m)}, k=1, \ldots,(\ell+m)$ by the following conditions

$$
\begin{aligned}
& \mathcal{H}_{k}^{(m, \ell+m)}\left(x, \partial_{x}\right) \cdot \Psi_{\underline{\lambda}}^{(m, \ell+m)}(x) \\
& \quad=\hbar^{k} \mathrm{e}^{-\rho_{1}\left(x_{1}-x_{\ell+m}\right)}\left\langle\psi_{\mathrm{L}}\left|\pi_{\underline{\lambda}}\left(C_{k} g(x)\right)\right| \psi_{\mathrm{R}}\right\rangle,
\end{aligned}
$$

where $C_{k} \in \mathcal{Z}\left(U \mathfrak{g l}_{\ell+m}\right)$ is a Casimir element of the center acting in $\mathcal{V}_{\underline{\lambda}}$ by multiplication on $k$ th elementary symmetric polynomial $\sigma_{k}(\underline{\lambda})$ of the variables $\lambda_{i}, i=1, \ldots,(\ell+m)$. Thus, Whittaker function $\Psi_{\lambda}^{(m, \overline{\ell+m})}(x)$ tautologically satisfy the following system of differential equations

$$
\begin{aligned}
& \mathcal{H}_{k}^{(m, \ell+m)}\left(x, \partial_{x}\right) \cdot \Psi_{\underline{\lambda}}^{(m, \ell+m)}(x) \\
& \quad=\sigma_{k}(\underline{\lambda}) \Psi_{\underline{\lambda}}^{(m, \ell+m)}(x), \quad k=1, \ldots,(\ell+m),
\end{aligned}
$$


Remark 1.1. The set of mutually commuting differential operators $\mathcal{H}_{k}^{(m, \ell+m)}, k=1, \ldots,(\ell+m)$ defines a quantum integrable system generalizing the standard quantum $\mathfrak{g l}_{\ell+m}$-Toda chain. The Whittaker functions $\Psi_{\underline{\lambda}}^{(m, \ell+m)}(x)$ provide a solution of the corresponding eigenfunction problem.

For the first two lowest degree differential operators $\mathcal{H}_{1}^{(m, \ell+m)}, \mathcal{H}_{2}^{(m, \ell+m)}$, we have

$$
\begin{aligned}
C_{1} & =\sum_{j=1}^{\ell+m} E_{j j}, \\
C_{2} & =\sum_{i<j}^{\ell+m}\left(E_{i i} E_{j j}-E_{j i} E_{i j}\right)-\sum_{j=1}^{\ell+m} \rho_{j} E_{j j}+\sigma_{2}(\rho),
\end{aligned}
$$

where $\rho=\left(\rho_{1}, \ldots, \rho_{\ell+m}\right) \quad$ with $\quad \rho_{k}=\frac{\ell+m+1}{2}-k, k=1, \ldots, \ell+m \quad$ and $\sigma_{2}(\rho)=\sum_{i<j} \rho_{i} \rho_{j}$. These operators act in the representation $\mathcal{V}_{\underline{\lambda}}$ via multiplication on

$$
\sigma_{1}(\underline{\lambda})=\sum_{j=1}^{\ell+m} \lambda_{j}, \quad \sigma_{2}(\underline{\lambda})=\sum_{1 \leq i<j \leq \ell+m} \lambda_{i} \lambda_{j} .
$$

The explicit form of the differential operators $\mathcal{H}_{1}$ and $\mathcal{H}_{2}$ corresponding to $C_{1}$ and $C_{2}$ is as follows.

Proposition 1.1. The following differential operators satisfy Equations (1.10)

$$
\begin{aligned}
\mathcal{H}_{1}^{(m, \ell+m)}\left(x, \partial_{x}\right)= & -\hbar \frac{\partial}{\partial x_{1}}-\hbar \frac{\partial}{\partial x_{\ell+m}}, \\
\mathcal{H}_{2}^{(m, \ell+m)}= & \hbar^{2}\left\{\frac{\partial^{2}}{\partial x_{1} \partial x_{\ell+m}}+\sum_{k=2}^{m} x_{k} \frac{\partial^{2}}{\partial x_{1} \partial x_{k}}-\sum_{2 \leq k \leq a}^{m} x_{k} x_{a} \frac{\partial^{2}}{\partial x_{k} \partial x_{a}}\right. \\
& -\sum_{n=1}^{\ell-1} x_{m+n} \frac{\partial^{2}}{\partial x_{m+n} \partial x_{\ell+m}}-\sum_{1 \leq n \leq b}^{\ell-1} x_{m+n} x_{m+b} \\
& \times \frac{\partial^{2}}{\partial x_{m+n} \partial x_{m+b}}+\sum_{k=2}^{m}\left(\rho_{k}\right) x_{k} \frac{\partial}{\partial x_{k}}
\end{aligned}
$$




$$
\begin{aligned}
& \left.-\sum_{n=1}^{\ell-1}\left(\rho_{m+n}\right) x_{m+n} \frac{\partial}{\partial x_{m+n}}\right\} \\
& +\hbar\left\{\left(1-\delta_{m, 1}\right) \frac{\partial}{\partial x_{2}}-\left(1-\delta_{\ell, 1}\right) \frac{\partial}{\partial x_{\ell+m-1}}\right. \\
& \left.+\sum_{k=2}^{m-1} x_{k} \frac{\partial}{\partial x_{k+1}}-\sum_{n=1}^{\ell-2} x_{m+n+1} \frac{\partial}{\partial x_{m+n}}\right\} \\
& +(-1)^{\delta_{\ell, 1}+\epsilon(\ell, m)}\left(x_{m}\right)^{1-\delta_{m, 1}}\left(x_{m+1}\right)^{1-\delta_{\ell, 1}} \mathrm{e}^{x_{1}-x_{\ell+m}} \\
& -\frac{\hbar^{2}}{24}(\ell+m-1)(\ell+m-2)(\ell+m-3) .
\end{aligned}
$$

Proof. The case of $\mathcal{H}_{1}^{(m, \ell+m)}$ is trivial and the proof of the expression for $\mathcal{H}_{2}^{(m, \ell+m)}$ is given in Appendix A.

Corollary 1.1. The $(m, \ell+m)$-parabolic Whittaker function (1.8) satisfies the following equations:

$$
\begin{aligned}
& \mathcal{H}_{1}^{(m, \ell+m)}\left(x, \partial_{x}\right) \Psi_{\underline{\lambda}}^{(m, \ell+m)}(x)=\sum_{j=1}^{\ell+m} \lambda_{j} \Psi_{\underline{\lambda}}^{(m, \ell+m)}(x), \\
& \mathcal{H}_{2}^{(m, \ell+m)}\left(x, \partial_{x}\right) \Psi_{\underline{\lambda}}^{(m, \ell+m)}(x)=\sum_{i<j}^{\ell+m} \lambda_{i} \lambda_{j} \Psi_{\underline{\lambda}}^{(m, \ell+m)}(x),
\end{aligned}
$$

where $\mathcal{H}_{1}^{(m, \ell+m)}\left(x, \partial_{x}\right)$ and $\mathcal{H}_{2}^{(m, \ell+m)}\left(x, \partial_{x}\right)$ are given by $(1.13)$.

Example 1.1. For $m=1$ the quadratic Hamiltonian (1.13) has the following form:

$$
\begin{aligned}
\mathcal{H}_{2}^{(1, \ell+1)}= & \hbar^{2} \frac{\partial^{2}}{\partial x_{1} \partial x_{\ell+1}}-\sum_{k=2}^{\ell} x_{k} \hbar^{2} \frac{\partial^{2}}{\partial x_{k} \partial x_{\ell+1}}-\sum_{2 \leq k \leq a}^{\ell} x_{k} x_{a} \hbar^{2} \frac{\partial^{2}}{\partial x_{k} \partial x_{a}} \\
& -\sum_{k=2}^{\ell}\left(\rho_{k}\right) x_{k} \hbar^{2} \frac{\partial}{\partial x_{k}}+\hbar \frac{\partial}{\partial x_{\ell}}-\hbar \sum_{j=2}^{\ell-1} x_{j+1} \frac{\partial}{\partial x_{j}} \\
& +(-1)^{\epsilon(\ell, 1)} x_{2} \mathrm{e}^{x_{1}-x_{\ell+1}}-\frac{\hbar^{2}}{24} \ell(\ell-1)(\ell-2) .
\end{aligned}
$$


For $\ell=1$ the quadratic Hamiltonian (1.13) reads as follows:

$$
\begin{aligned}
\mathcal{H}_{2}^{(m, m+1)}= & \hbar^{2} \frac{\partial^{2}}{\partial x_{1} \partial x_{m+1}}+\sum_{k=2}^{m} x_{k} \hbar^{2} \frac{\partial^{2}}{\partial x_{1} \partial x_{k}}-\sum_{2 \leq k \leq a}^{m} x_{k} x_{a} \hbar^{2} \frac{\partial^{2}}{\partial x_{k} \partial x_{a}} \\
& +\sum_{k=2}^{m}\left(\rho_{k}\right) x_{k} \hbar^{2} \frac{\partial}{\partial x_{k}}+\hbar \frac{\partial}{\partial x_{2}}+\hbar \sum_{k=2}^{m-1} x_{k} \frac{\partial}{\partial x_{k+1}} \\
& -(-1)^{\epsilon(m, 1)} x_{m} \mathrm{e}^{x_{1}-x_{m+1}}-\frac{\hbar^{2}}{24} m(m-1)(m-2) .
\end{aligned}
$$

We conjecture that $(m, \ell+m)$-parabolic Whittaker functions describe equivariant Gromov-Witten invariants of the Grassmannian $\operatorname{Gr}(m, \ell+m)=$ $G L_{\ell+1}(\mathbb{C}) / P_{m, \ell+m}$ thus generalizing the Givental description [21] of $S^{1} \times$ $U_{\ell+1}$-equivariant Gromov-Witten invariants of the complete flag spaces $G L_{\ell+1}(\mathbb{C}) / B$. We support our conjecture by matching it with a description of quantum cohomology of $\operatorname{Gr}(m, \ell+m)$ due to Astashkevich-Sadov-Kim $[2,27]$. To establish a relation with $[2,27]$ let us define a quantum $\mathcal{L}$-operator associated with the quantum integrable system (1.10) as a matrix-valued differential operator satisfying the relation

$$
\begin{aligned}
& \mathcal{L}\left(x, \partial_{x}\right) \mathrm{e}^{-\rho_{1}\left(x_{1}-x_{\ell+m}\right)}\left\langle\psi_{\mathrm{L}}\left|\pi_{\underline{\lambda}}(g(x))\right| \psi_{\mathrm{R}}\right\rangle \\
& \quad:=\hbar \sum_{i, j=1}^{\ell+1} e_{i j} \mathrm{e}^{-\rho_{1}\left(x_{1}-x_{\ell+m}\right)}\left\langle\psi_{\mathrm{L}}\left|\pi_{\underline{\lambda}}\left(E_{i j} g(x)\right)\right| \psi_{\mathrm{R}}\right\rangle,
\end{aligned}
$$

where $\left(e_{i j}\right)_{k n}=\delta_{i k} \delta_{j n}, i, j, k, n=1, \ldots,(\ell+m)$ are matrix unites.

Proposition 1.2. The matrix $\mathcal{L}=\left\|\mathcal{L}_{i j}\right\|$ of the quantum L-operator $(1.17)$ is given by

$$
\begin{gathered}
\mathcal{L}_{j+1, j}=1, \quad 1 \leq j \leq \ell+m-1, \\
\mathcal{L}_{j+s, j}=0, \quad 1 \leq j \leq \ell+m-1, \quad 2 \leq s \leq \ell+m-j, \\
\mathcal{L}_{11}=-\hbar \partial_{x_{1}}-\hbar \frac{\ell+m-1}{2}+\sum_{k=2}^{m} x_{k} \hbar \partial_{x_{k}}, \quad \mathcal{L}_{1, k}=-\hbar \partial_{x_{k}}, \quad 2 \leq k \leq m, \\
\mathcal{L}_{k, k+j}=-x_{k} \hbar \partial_{x_{k+j}}, \quad 2 \leq k \leq m, 0 \leq j \leq m-k, \\
\mathcal{L}_{1, m+j}=-(-1)^{\epsilon(\ell, m)} x_{m+j} \mathrm{e}^{x_{1}-x_{\ell+m}}, \quad 1 \leq j \leq \ell-1,
\end{gathered}
$$




$$
\begin{gathered}
\mathcal{L}_{1, \ell+m}=(-1)^{\epsilon(\ell, m)} \mathrm{e}^{x_{1}-x_{\ell+m}}, \\
\mathcal{L}_{k, m+j}=-(-1)^{\epsilon(\ell, m)} x_{k} x_{m+j} \mathrm{e}^{x_{x_{1}-\ell+m}}, \quad 2 \leq k \leq m, \quad 1 \leq j \leq \ell-1, \\
\mathcal{L}_{k, \ell+m}=(-1)^{\epsilon(\ell, m)} x_{k} \mathrm{e}^{x_{1}-x_{\ell+m}}, \quad 2 \leq k \leq m, \\
\mathcal{L}_{m+n, \ell+m}=-\hbar \partial_{x_{m+n}}, \quad 1 \leq n \leq \ell-1, \\
\mathcal{L}_{m+n, m+n+j}=x_{m+n+j} \hbar \partial_{x_{m+n}}, \quad 1 \leq n \leq \ell-2, \quad 0 \leq j \leq \ell-n-1, \\
\mathcal{L}_{\ell+m, \ell+m}=-\hbar \partial_{x_{\ell+m}}+\hbar \frac{\ell+m-1}{2}-\sum_{k=1}^{\ell-1} x_{m+k} \hbar \partial_{x_{m+k}} .
\end{gathered}
$$

Proof. The proof is given in Appendix B.

The classical limit $L$ of the operator (1.17) is defined by replacing derivatives by the classical momenta $-\hbar \frac{\partial}{\partial x_{j}} \rightarrow p_{j}$ and taking the limit $\hbar \rightarrow 0$. Let us specialize the resulting matrix function $L\left(x_{1}, \ldots, x_{\ell+m} ; p_{1}, \ldots, p_{\ell+m}\right)$ by taking $x_{2}=\cdots=x_{\ell+m-1}=0$. This way we obtain the matrix $L=\left\|L_{i j}\right\|$ with the following entries:

$$
\begin{gathered}
L_{j+1, j}=1, \quad 1 \leq j \leq \ell+m-1, \\
L_{1, k}=p_{k}, \quad 1 \leq k \leq m, \\
L_{m+n, \ell+m}=p_{m+n}, \quad 1 \leq n \leq \ell, \\
L_{1, \ell+m}=(-1)^{\epsilon(\ell, m)} \mathrm{e}^{x_{1}-x_{\ell+m}} .
\end{gathered}
$$

It is easy to verify that thus defined matrix $L=\left\|L_{i j}\right\|$ coincides (up to a conjugation by a simple matrix) with the matrix entering a description of small quantum cohomology of $\operatorname{Gr}(m, \ell+m)[2,27]$. This supports the conjecture that $(m, \ell+m)$-Whittaker functions are relevant to a description of $S^{1} \times U_{\ell+m}$-equivariant quantum cohomology of $\operatorname{Gr}(m, \ell+m)$.

In the rest of the note we will consider only the case of $m=1$ and arbitrary $\ell$. In this case, the conjectural relation between solutions of the generalized Toda chain given by $(1, \ell+1)$-Whittaker functions and $S^{1} \times$ $U_{\ell+m}$-equivariant Gromov-Witten invariants of $\mathbb{P}^{\ell}=G L_{\ell+1} / P_{1, \ell+1}$ can be proved as follows. For $m=1$, there is a well-known description of $S^{1} \times$ $U_{\ell+1}$-equivariant quantum cohomology of $G r(1, \ell+1)=\mathbb{P}^{\ell}$ in terms of the functions given by the following integral expressions (see, e.g. [19]). Let us introduce a modified $\Gamma$-function

$$
\Gamma_{1}(z \mid \omega)=\omega^{\frac{z}{\omega}} \Gamma\left(\frac{z}{\omega}\right)
$$


Then

$$
\begin{aligned}
\Phi_{\underline{\lambda}}(x)= & \int_{\mathcal{C}} \prod_{j=1}^{\ell} d t_{j} \exp \left(-\frac{1}{\hbar}\left(\sum_{j=1}^{\ell} \lambda_{j} t_{j}+\lambda_{\ell+1}\left(x-\sum_{j=1}^{\ell} t_{j}\right)\right.\right. \\
& \left.\left.+\sum_{j=1}^{\ell} \mathrm{e}^{t_{j}}+\mathrm{e}^{x-\sum_{j=1}^{\ell} t_{j}}\right)\right) \\
= & \left.\frac{1}{2 \pi \hbar} \int_{\mathbb{R}-\imath \epsilon} d H \mathrm{e}^{-\frac{\imath}{\hbar} x H} \prod_{j=1}^{\ell+1} \Gamma_{1}\left(\imath H-\lambda_{j}\right) \mid \hbar\right)
\end{aligned}
$$

satisfies the differential equation

$$
\left(\prod_{j=1}^{\ell+1}\left(-\hbar \partial_{x}-\lambda_{j}\right)-\mathrm{e}^{x}\right) \Phi_{\underline{\lambda}}(x)=0
$$

Here $\epsilon>\max \left(\lambda_{j}\right) j=1, \ldots, \ell+1$ and $\mathcal{C}$ is a slightly deformed subspace $\mathbb{R}^{\ell} \subset \mathbb{C}^{\ell}$ making the integral (1.21) convergent. For $\ell=1$ the function (1.21) is the classical $\mathfrak{g l}_{2}$-Whittaker function.

Theorem 1.1. The $(1, \ell+1)$-parabolic Whittaker function specialized to $x=x_{1}$ and $x_{i}=0, i \neq 1$ and for $\epsilon(1, \ell+1)=\frac{\ell(\ell-1)}{2}+1$ coincides with the generating function (1.21) of $S^{1} \times U_{\ell+1}$-equivariant quantum cohomology of $\mathbb{P}^{\ell}$. That is

$$
\Psi_{\underline{\lambda}}^{(1, \ell+1)}(x, 0, \ldots, 0)=\frac{1}{2 \pi \hbar} \int_{\mathbb{R}-\imath \epsilon} d H \mathrm{e}^{-\frac{\imath}{\hbar} x H} \prod_{j=1}^{\ell+1} \Gamma_{1}\left(\imath H-\lambda_{j} \mid \hbar\right),
$$

where $\underline{\lambda} \in \mathbb{R}^{\ell+1}$ and $\epsilon>\max \left(\lambda_{j}\right), j=1, \ldots, \ell+1$.

Proof. The proof is given in Appendix C.

The integral representation (1.22) arises naturally when the matrix element (1.8) for $m=1$ is represented by using the Gelfand-Zetlin realizations of the infinite-dimensional representations of $\mathcal{U} \mathfrak{g l}_{\ell+1}$ [13] (similar relation holds for the integral representation (1.21) and the representation of $\mathcal{U}_{\mathfrak{g l}_{\ell+1}}$ constructed in [14]). Note that one has an obvious symmetry $G r(m, \ell+m)=$ $G r(\ell, \ell+m)$. The compatibility of our conjecture with this isomorphism is explicitly checked for $m=1, \ell=2$ in Appendix D. 
In the next sections, we propose an identification of the generating functions given by the integral representations (1.22), (1.21) with particular correlation functions in type- $A$ and type- $B$ equivariant two-dimensional topological sigma models on a disk.

\section{Type-A topological sigma model with a target space $\mathbb{P}^{\ell}$}

In this section, we define a class of correlation functions in $S^{1} \times U_{\ell+1^{-}}$ equivariant type- $A$ topological sigma model on a disk with a target space $\mathbb{P}^{\ell}$ and calculate the correlation functions explicitly. The resulting expressions coincide with the integrals (1.21). This provides an infinite-dimensional integral representations of $(1, \ell+1)$-Whittaker functions $(1.8)$ in terms of topological type- $A$ equivariant sigma models on a disk. As a background material for two-dimensional topological field theories and functional integration of the Gaussian measures over linear superspaces see e.g. [11].

\subsection{Topological gauged linear sigma model}

We start recalling a gauge linear sigma model realization of the sigma-model with the target space $\mathbb{P}^{\ell}$ (see, e.g. $[29,34,35]$ ). Consider a type- $A$ topological linear sigma model on a Riemann surface $\Sigma$ with the target space $\mathbb{C}^{\ell+1}$. Let $(z, \bar{z})$ be local complex coordinates on $\Sigma$. We pick a Hermitian metric $h$ on $\Sigma$ and denote $\sqrt{h} d^{2} z$ the corresponding measure on $\Sigma$. The complex structure defines a decomposition $d=\partial+\bar{\partial}, \partial=d z \partial_{z}, \bar{\partial}=d \bar{z} \partial_{\bar{z}}$ of the de Rham differential $d$ acting on differential forms on $\Sigma$. Let $K$ and $\bar{K}$ be canonical and anti-canonical bundles over $\Sigma$. Let $T_{\mathbb{C}} \mathbb{C}^{\ell+1}=T^{1,0} \mathbb{C}^{\ell+1} \oplus T^{0,1} \mathbb{C}^{\ell+1}$ be a decomposition of the (trivial) complexified tangent bundle to $\mathbb{C}^{\ell+1}$ induced by a standard complex structure on $\mathbb{C}^{\ell+1}$. We denote linear complex coordinates on $\mathbb{C}^{\ell+1}$ by $\left(\varphi^{j}, \bar{\varphi}^{j}\right)$. Consider a two-dimensional topological quantum filed theory based in the maps $\Phi: \Sigma \rightarrow \mathbb{C}^{\ell+1}$ with the action functional

$$
\begin{aligned}
S & =\int_{\Sigma} d^{2} z \sqrt{h} \delta_{0} \mathcal{V} \\
& =\int_{\Sigma} d^{2} z h^{z \bar{z}} \sqrt{h} \sum_{j=1}^{\ell+1}\left(t \bar{F}_{z}^{j} F_{\bar{z}}^{j}+\imath \bar{F}_{z}^{j} \partial_{\bar{z}} \varphi^{j}-\imath F_{\bar{z}}^{j} \partial_{z} \bar{\varphi}^{j}+\imath \psi_{\bar{z}}^{j} \partial_{z} \bar{\chi}^{j}-\imath \bar{\psi}_{z}^{j} \partial_{\bar{z}} \chi^{j}\right),
\end{aligned}
$$


where

$$
\mathcal{V}=h^{z \bar{z}} \sum_{j=1}^{\ell+1}\left(\bar{\psi}_{z}^{j}\left(\frac{t}{2} F_{\bar{z}}^{j}+\imath \partial_{\bar{z}} \varphi^{j}\right)+\psi_{\bar{z}}^{j}\left(\frac{t}{2} \bar{F}_{z}^{j}-\imath \partial_{z} \bar{\varphi}^{j}\right)\right),
$$

and BRST [11] transformations are given by

$$
\begin{array}{llll}
\delta_{0} \varphi^{j}=\chi^{j}, & \delta_{0} \chi^{j}=0, & \delta_{0} \psi_{\bar{z}}^{j}=F_{\bar{z}}^{j}, & \delta_{0} F_{\bar{z}}^{j}=0, \\
\delta_{0} \bar{\varphi}^{j}=\bar{\chi}^{j}, & \delta_{0} \bar{\chi}^{j}=0, & \delta_{0} \bar{\psi}_{z}^{j}=\bar{F}_{z}^{j}, & \delta_{0} \bar{F}_{z}^{j}=0 .
\end{array}
$$

Here the commuting fields $F$ and $\bar{F}$ are sections of $K \otimes \Phi^{*}\left(T^{0,1} \mathbb{C}^{\ell+1}\right)$ and of $\bar{K} \otimes \Phi^{*}\left(T^{1,0} \mathbb{C}^{\ell+1}\right)$ correspondingly. The anticommuting fields $\chi, \bar{\chi}$ are sections of the bundles $\Phi^{*}\left(\Pi T^{1,0} \mathbb{C}^{\ell+1}\right), \Phi^{*}\left(\Pi T^{0,1} \mathbb{C}^{\ell+1}\right)$ and anticommuting fields $\psi, \bar{\psi}$ are sections of the bundles $K \otimes \Phi^{*}\left(\Pi T^{0,1} \mathbb{C}^{\ell+1}\right), \bar{K} \otimes \Phi^{*}\left(\Pi T^{1,0}\right.$ $\left.\mathbb{C}^{\ell+1}\right)$. By $\Pi \mathcal{E}$ we denote a vector bundle $\mathcal{E}$ with a reversed parity of the fibres. The action (2.1) is $\delta_{0}$-invariant.

A gauged linear sigma model description of the sigma model with the target space $\mathbb{P}^{\ell}$ is based on the representation of the projective space $\mathbb{P}^{\ell}$ as a Hamiltonian quotient of $\mathbb{C}^{\ell+1}$. Let us supply $\mathbb{C}^{\ell+1}$ with a symplectic structure

$$
\Omega=\frac{\imath}{2} \sum_{j=1}^{\ell+1} d \varphi^{j} \wedge d \bar{\varphi}^{j} .
$$

The following action of $U_{1}$ :

$$
\varphi^{j} \longrightarrow \mathrm{e}^{\imath \alpha} \varphi^{j}, \quad \mathrm{e}^{\imath \alpha} \in U(1)
$$

is Hamiltonian and the corresponding momentum, i.e., a solution of the equation

$$
\iota_{v} \Omega=d \mu
$$

is given by

$$
\mu(\varphi, \bar{\varphi})=-\frac{1}{2} \sum_{j=1}^{\ell+1}\left|\varphi^{j}\right|^{2} .
$$

The projective space $\mathbb{P}^{\ell}$ has the following representation as the Hamiltonian quotient:

$$
\mathbb{P}^{\ell}=\left\{\mu(\varphi, \bar{\varphi})+r^{2} / 2=0\right\} / U(1) .
$$

where the value $r^{2} / 2$ of the momentum $\mu$ defines a Kähler class of $\mathbb{P}^{\ell}$. 
The interpretation of the projective space as a Hamiltonian reduction allows to describe topological non-linear two-dimensional sigma model with the target space $\mathbb{P}^{\ell}$ in terms of a linear sigma model with the target space $\mathbb{C}^{\ell+1}$ and gauged $U_{1}$-symmetry $(2.4)$. Topological $U_{1}$ gauge theory can be constructed using the following three sets of fields $(A, \lambda, \sigma),(b, \eta),(\xi, H)$, where $A$ is a connection in $U_{1}$-bundle, $\psi$ is an odd one-form, $\sigma, b, H$ are real even zero forms and $\xi, \eta$ are real odd zero forms. Define the topological BRST transformations as follows

$$
\begin{aligned}
& \delta_{\mathcal{G}} A=\lambda, \quad \delta_{\mathcal{G}} \lambda=-\imath d \sigma, \quad \delta_{\mathcal{G}} \sigma=0, \\
& \delta_{\mathcal{G}} b=\eta, \quad \delta_{\mathcal{G}} \eta=0, \quad \delta_{\mathcal{G}} \xi=H, \quad \delta_{\mathcal{G}} H=0 .
\end{aligned}
$$

Note that $\delta_{\mathcal{G}}^{2}$ acts as a gauge transformation with the gauge parameter $\sigma$ e.g.

$$
\delta_{\mathcal{G}}^{2} A=-\imath d \sigma, \quad \delta_{\mathcal{G}}^{2} \lambda=0, \quad \delta_{\mathcal{G}}^{2} \sigma=0
$$

The space of functionals of the fields $(A, \lambda, \sigma)$ supplied with the action of $\delta_{\mathcal{G}}$ can be considered as a model for the $\mathcal{G}$-equivariant de Rham complex on the space of connections on a Riemann surface $\Sigma$. In the following, we consider the gauge multiplet $(A, \lambda, \sigma)$ interacting with the fields $(b, \eta),(\xi, H)$ and with the fields entering the action (2.1). The gauge theory generalization $\delta_{\mathcal{G}}$ of the BRST transformation (2.2) acts as follows

$$
\begin{aligned}
& \delta_{\mathcal{G}} \varphi^{j}=\chi^{j}, \quad \delta_{\mathcal{G}} \chi^{j}=-\imath \sigma \varphi^{j}, \quad \delta_{\mathcal{G}} \psi_{\bar{z}}^{j}=F_{\bar{z}}^{j}, \quad \delta_{\mathcal{G}} F_{\bar{z}}^{j}=-\imath \sigma \psi_{\bar{z}}^{j}, \\
& \delta_{\mathcal{G}} \bar{\varphi}^{j}=\bar{\chi}^{j}, \quad \delta_{\mathcal{G}} \bar{\chi}^{j}=\imath \sigma \bar{\varphi}^{j}, \quad \delta_{\mathcal{G}} \bar{\psi}_{z}^{j}=\bar{F}_{z}^{j}, \quad \delta_{\mathcal{G}} \bar{F}_{z}^{j}=\imath \sigma \bar{\psi}_{z}^{j} .
\end{aligned}
$$

Let $* d z=\imath d z$ and $* d \bar{z}=-\imath d \bar{z}$ be the Hodge star operators. A topological $U_{1}$-gauged linear sigma model is described by the following action:

$$
\begin{aligned}
S= & \frac{1}{2 \pi} \int_{\Sigma} d^{2} z \sqrt{h} \delta_{\mathcal{G}} \mathcal{V}=\frac{1}{2 \pi \mathrm{e}^{2}} \int_{\Sigma} d^{2} z \sqrt{h} h^{z \bar{z}}\left(-H F_{z \bar{z}}(A)+\partial_{z} \lambda_{\bar{z}}(\xi-\imath \eta)\right. \\
& \left.-\partial_{\bar{z}} \lambda_{z}(\xi+\imath \eta)-2 b \partial_{z} \partial_{\bar{z}} \sigma\right)+\frac{1}{2 \pi} \int_{\Sigma} d^{2} z \sqrt{h} h^{z \bar{z}}\left(t \bar{F}_{z}^{j} F_{\bar{z}}^{j}+\imath \bar{F}_{z}^{j}\left(\partial_{\bar{z}}-A_{\bar{z}}\right) \varphi^{j}\right. \\
& -\imath F_{\bar{z}}^{j}\left(\partial_{z}-A_{z}\right) \bar{\varphi}^{j}+\imath t \psi_{\bar{z}}^{j} \sigma \bar{\psi}_{z}^{j}-\imath \bar{\psi}_{z}^{j}\left(\partial_{\bar{z}} \chi^{j}-A_{\bar{z}} \chi^{j}\right)+\imath \psi_{\bar{z}}^{j}\left(\partial_{z} \bar{\chi}^{j}-A_{\bar{z}} \bar{\chi}^{j}\right)
\end{aligned}
$$




$$
\begin{aligned}
& -\imath \psi_{\bar{z}}^{j} \lambda_{z} \bar{\varphi}^{j}+\imath \bar{\psi}_{z}^{j} \lambda_{\bar{z}} \varphi^{j}+\eta \sum_{j=1}^{\ell+1}\left(\varphi^{j} \bar{\chi}^{j}-\bar{\varphi}^{j} \chi^{j}\right)+2 b \sum_{j=1}^{\ell+1}\left(\chi^{j} \bar{\chi}^{j}+\imath \sigma\left|\varphi^{j}\right|^{2}\right) \\
& +\frac{\imath H}{2}\left(\sum_{j=1}^{\ell+1}|\varphi|^{2}-r^{2}\right)-\frac{\imath \xi}{2}\left(\sum_{j=1}^{\ell+1} \chi^{j} \bar{\varphi}^{j}+\bar{\chi}^{j} \varphi^{j}\right)
\end{aligned}
$$

where $F_{z \bar{z}}(A)=\partial_{z} A_{\bar{z}}-\partial_{\bar{z}} A_{z}$ and

$$
\begin{aligned}
\mathcal{V}= & \frac{1}{\mathrm{e}^{2}} h^{z \bar{z}}\left(-\xi F_{z \bar{z}}(A)-\imath b\left(\partial_{z} \lambda_{\bar{z}}+\partial_{\bar{z}} \lambda_{z}\right)\right)+\psi_{\bar{z}}^{j}\left(\frac{t}{2} \bar{F}_{z}^{j}-\imath\left(\partial_{z}-A_{z}\right) \bar{\varphi}^{j}\right) \\
& +\bar{\psi}_{z}^{j}\left(\frac{t}{2} F_{\bar{z}}^{j}+\imath\left(\partial_{\bar{z}}-A_{\bar{z}}\right) \varphi^{j}\right)+\frac{\imath \xi}{2}\left(\sum_{j=1}^{\ell+1}|\varphi|^{2}-r^{2}\right)+b \sum_{j=1}^{\ell+1}\left(\varphi^{j} \bar{\chi}^{j}-\bar{\varphi}^{j} \chi^{j}\right) .
\end{aligned}
$$

A relation of (2.9) (for a generic coupling constant $\mathrm{e}^{2}$ ) with the standard $\mathbb{P}^{\ell}$ sigma model is illustrated in Appendix F. Let us note that a variation of the coupling constants $\mathrm{e}^{2}$ and $t$ leads to a change of the action $(2.9)$ on a $\delta_{\mathcal{G}^{-}}$ exact term. Thus, according to the standard considerations, the dependence of correlation functions of $\delta_{\mathcal{G}}$-closed operators on $\mathrm{e}^{2}$ and $t$ is given only by the boundary contributions of the space of fields. In many cases these boundary contributions are zero and the correlation functions are $\mathrm{e}^{2}$ - and $t$-independent. In the following, we make calculations at particular values of the coupling constants $\mathrm{e}^{2}$ and $t$ and will not consider the issue of the coupling constant independence.

Now we consider $S^{1} \times U_{\ell+1}$-equivariant version of the theory (2.9) on a Riemann surface $\Sigma$ allowing isometry $S^{1}$. The action of $U_{\ell+1}$ is induced from a linear action on the target space $\mathbb{C}^{\ell+1}$ and the action of $S^{1}$ is induced by $S^{1}$-isometry of $\Sigma$. To simplify notations we consider equivariance with respect to a maximal abelian subgroup $U_{1}^{\ell+1} \subset U_{\ell+1}$ and will work with functions invariant with respect to the permutation group $S_{\ell+1}$ (i.e., Weyl group of $\left.U_{\ell+1}\right)$. Let $\hbar$ and $\sigma_{j}, j=1, \ldots,(\ell+1)$ be generators of abelian Lie algebras $\operatorname{Lie}\left(S^{1}\right)$ and $\operatorname{Lie}\left(U_{1}^{\ell+1}\right)$. Correlation functions of $S^{1} \times U_{\ell+1^{-}}$-equivariant extension of the topological field theory (2.9) now take values in the space of functions of $\hbar$ and $\sigma_{j}, j=1, \ldots,(\ell+1)$ invariant with respect to permutations of $\sigma_{j}$.

To construct an $S^{1} \times U_{\ell+1^{-}}$-equivariant version of type- $A$ topological linear sigma model define $S^{1} \times U_{1}^{\ell+1}$-equivariant generalization of the BRST 
transformations (2.8) and (2.6) as follows:

$$
\begin{aligned}
& \delta \varphi^{j}=\chi^{j}, \quad \delta \chi^{j}=-\left(\imath\left(\sigma_{j}+\sigma\right) \varphi^{j}+\hbar \mathcal{L}_{v_{0}} \varphi^{j}\right), \quad \delta \psi^{j}=F^{j}, \\
& \delta F^{j}=-\left(\imath\left(\sigma_{j}+\sigma\right) \psi^{j}+\hbar \mathcal{L}_{v_{0}} \psi^{j}\right), \\
& \delta \bar{\varphi}^{j}=\bar{\chi}^{j}, \quad \delta \bar{\chi}^{j}=-\left(-\imath\left(\sigma_{j}+\sigma\right) \bar{\varphi}^{j}+\hbar \mathcal{L}_{v_{0}} \bar{\varphi}^{j}\right), \quad \delta \bar{\psi}^{j}=\bar{F}^{j}, \\
& \delta \bar{F}^{j}=-\left(-\imath\left(\sigma_{j}+\sigma\right) \bar{\psi}^{j}+\hbar \mathcal{L}_{v_{0}} \bar{\psi}^{j}\right), \\
& \delta A=\lambda, \quad \delta \lambda=-\imath d \sigma+\hbar d\left(\iota_{v_{0}} A\right)+\hbar \iota_{v_{0}} F(A), \quad \delta \sigma=0, \\
& \delta b=\eta, \quad \delta \eta=\hbar \iota_{v_{0}} d b, \quad \delta \xi=H, \quad \delta H=\hbar \iota_{v_{0}} d \xi,
\end{aligned}
$$

where $v_{0}=\imath\left(z \frac{\partial}{\partial z}-\bar{z} \frac{\partial}{\partial \bar{z}}\right)$ and $\mathcal{L}_{v_{0}}=\iota_{v_{0}} d+d \iota_{v_{0}}$. Note that the transformation rules (2.10) are not explicitly gauge-invariant (this is related with the fact that the gauge group $\mathcal{G}$ and $S^{1}$ do not commute).

A $S^{1} \times U_{1}^{\ell+1}$-equivariant version of the topological gauged linear sigma model (2.9) (for $t=0$ ) is then given by

$$
\begin{aligned}
S_{e q}= & \frac{1}{2 \pi} \int_{\Sigma} d^{2} z \sqrt{h} \delta_{2} \mathcal{V}=\frac{1}{2 \pi \mathrm{e}^{2}} \int_{\Sigma} d^{2} z \sqrt{h} h^{z \bar{z}}\left(\left(-H F_{z \bar{z}}(A)+(\lambda \wedge d \xi)_{z \bar{z}}\right.\right. \\
& \left.+(\lambda \wedge * d \eta)_{z \bar{z}}-\imath(d b \wedge * d \sigma)_{z \bar{z}}-\hbar b d *\left(\iota_{v_{0}} A+\iota_{v_{0}} F(A)\right)_{z \bar{z}}\right) \\
& +\frac{1}{2 \pi} \int_{\Sigma} d^{2} z \sqrt{h} h^{z \bar{z}}\left(\imath \bar{F}_{z}^{j}\left(\partial_{\bar{z}}-A_{\bar{z}}\right) \varphi^{j}-\imath F_{\bar{z}}^{j}\left(\partial_{z}-A_{z}\right) \bar{\varphi}^{j}\right. \\
& -\imath \bar{\psi}_{z}^{j}\left(\partial_{\bar{z}} \chi^{j}-A_{\bar{z}} \chi^{j}\right)+\imath \psi_{\bar{z}}^{j}\left(\partial_{z} \bar{\chi}^{j}-A_{z} \bar{\chi}^{j}\right)-\imath \psi_{\bar{z}}^{j} \lambda_{z} \bar{\varphi}^{j}+\imath \bar{\psi}_{z}^{j} \lambda_{\bar{z} \varphi^{j}} \\
& +\eta \sum_{j=1}^{\ell+1}\left(\varphi^{j} \bar{\chi}^{j}-\bar{\varphi}^{j} \chi^{j}\right)+b \sum_{j=1}^{\ell+1}\left(2 \chi^{j} \bar{\chi}^{j}+2 \imath\left(\sigma+\sigma_{j}\right)\left|\varphi^{j}\right|^{2}{ }^{\ell}{ }^{\ell+1} \sum_{j=1}|\varphi|^{2}-r^{2}\right) \\
& \left.\left.+\hbar \bar{\varphi}^{j} \iota_{v_{0}}(d-A) \varphi^{j}-\hbar \varphi^{j} \iota_{v_{0}}(d-A) \bar{\varphi}^{j}\right)+\frac{\imath H}{2}\left(\sum_{j=1}^{\ell+1} \chi^{j} \bar{\varphi}^{j}+\bar{\chi}^{j} \varphi^{j}\right)\right) \\
& -\frac{\imath \xi}{2}\left(\sum^{\ell}\right)
\end{aligned}
$$

where $F_{z \bar{z}}(A)=\partial_{z} A_{\bar{z}}-\partial_{\bar{z}} A_{z}$ and

$$
\begin{aligned}
\mathcal{V}= & \frac{1}{\mathrm{e}^{2}} h^{z \bar{z}}\left(-\xi F_{z \bar{z}}(A)+b(d * \lambda)_{z \bar{z}}\right)-\imath \psi_{\bar{z}}^{j}\left(\partial_{z}-A_{z}\right) \bar{\varphi}^{j}+\imath \bar{\psi}_{z}^{j}\left(\partial_{\bar{z}}-A_{\bar{z}}\right) \varphi^{j} \\
& +\frac{\imath \xi}{2}\left(\sum_{j=1}^{\ell+1}|\varphi|^{2}-r^{2}\right)+b \sum_{j=1}^{\ell+1}\left(\varphi^{j} \bar{\chi}^{j}-\bar{\varphi}^{j} \chi^{j}\right) .
\end{aligned}
$$




\subsection{Calculations in type-A topological sigma model on a disk}

Now we calculate a particular correlation function in a topological field theory with the action $(2.11)$ on a disk $\Sigma=D, D=\left\{\left.z \in \mathbb{C}|| z\right|^{2} \leq 1\right\}$ with the coupling constant $\mathrm{e}^{2} \rightarrow 0$. We chose a flat metric $d^{2} s=|d z|^{2}$ on the disk $D$ invariant with respect to the standard action of the rotation group $S^{1}$ generated by the vector field

$$
v_{0}=\imath\left(z \frac{\partial}{\partial z}-\bar{z} \frac{\partial}{\partial \bar{z}}\right), \quad z=r \mathrm{e}^{\imath \theta}
$$

The following boundary conditions on $S^{1}=\partial D$ are imposed:

$$
\begin{aligned}
& \left.A_{\theta}\right|_{S^{1}}=0,\left.\quad \lambda_{\theta}\right|_{S^{1}}=0,\left.\quad \partial_{\theta} \sigma\right|_{S^{1}}=0, \\
& \left.\partial_{\theta} b\right|_{S^{1}}=0,\left.\quad \partial_{\theta} \xi\right|_{S^{1}}=0,\left.\quad \partial_{\theta} \eta\right|_{S^{1}}=0 .
\end{aligned}
$$

We also consider a restricted gauge group

$$
\mathcal{G}_{0}=\left\{g \in \operatorname{Map}\left(D, U_{1}\right)|g|_{S^{1}}=\text { const }\right\}
$$

It is easy to see that the deformed action (2.11) and the boundary conditions are compatible with the gauge symmetry $\mathcal{G}_{0}$ and BRST transformations (2.10). For example, we have

$$
\delta A_{\theta}=\lambda_{\theta}, \quad \delta \lambda_{\theta}=\partial_{\theta} \sigma+\hbar \partial_{\theta} A_{\theta}
$$

and restriction of the variations to the boundary $S^{1}=\partial D$ gives

$$
\left.\delta A_{\theta}\right|_{S^{1}}=\left.\lambda_{\theta}\right|_{S^{1}}=0,\left.\quad \delta \lambda_{\theta}\right|_{S^{1}}=\left.\partial_{\theta} \sigma\right|_{S^{1}}+\left.\hbar \partial_{\theta} A_{\theta}\right|_{S^{1}}=0
$$

This verifies the compatibility of the boundary conditions (2.12) and BRST transformations for the gauge multiplet. Similarly, under infinitesimal gauge transformations we have

$$
\left.\delta_{\alpha} A_{\theta}\right|_{S^{1}}=\left.\partial_{\theta} \alpha\right|_{S^{1}}+\left.A_{\theta}\right|_{S^{1}}=0, \quad \alpha \in \operatorname{Lie}\left(\mathcal{G}_{0}\right),
$$

and thus the boundary conditions (2.12) are compatible with gauge invariance with respect to group (2.14). We define a metric on the space of fields using the standard quadratic form on the tensor fields on $D$. 
Proposition 2.1. A functional integral over fields on the disk with the action (2.11) at $\mathrm{e}^{2} \rightarrow 0$ has an asymptotic given by a functional integral with the effective action on the boundary $\partial D=S^{1}$

$$
\begin{aligned}
S_{\mathrm{eff}}= & \frac{\imath H_{0}}{4 \pi} \int_{0}^{2 \pi} d \theta\left(\sum_{j=1}^{\ell+1}\left|\varphi^{j}(\theta)\right|^{2}-r^{2}\right)-\frac{\imath \xi_{0}}{4 \pi} \int_{0}^{2 \pi} d \theta \sum_{j=1}^{\ell+1}\left(\bar{\varphi}^{j}(\theta) \chi^{j}(\theta)+\varphi^{j}(\theta) \bar{\chi}^{j}(\theta)\right) \\
& +\frac{\eta_{0}}{2 \pi} \int_{0}^{2 \pi} d \theta \sum_{j=1}^{\ell+1}\left(\varphi^{j}(\theta) \bar{\chi}^{j}(\theta)-\bar{\varphi}^{j}(\theta) \chi^{j}(\theta)\right) \\
& +2 b_{0} \sum_{j=1}^{\ell+1} \frac{1}{2 \pi} \int_{0}^{2 \pi} d \theta\left[\chi^{j}(\theta) \bar{\chi}^{j}(\theta)+\imath\left(\sigma_{0}+\sigma_{j}\right)\left|\varphi^{j}(\theta)\right|^{2}+\hbar \bar{\varphi}^{j}(\theta) \partial_{\theta} \varphi^{j}(\theta)\right] .
\end{aligned}
$$

Here in the functional integral over $\varphi^{j}(z), \chi^{j}(z)$ being even and odd holomorphic functions on $D, \sigma_{0}$ and $b_{0}$ being even variables, $\xi_{0}$ and $\eta_{0}$ being odd variables. The measure on the space of $\varphi^{j}(z)$ and $\chi^{j}(z)$ is induced from the standard pairing of functions on $D$ with the metric $d^{2} s=|d z|^{2}$.

Proof. We will need an asymptotic at $e^{2} \rightarrow 0$ of the various integrals. Given a function $F(e, y)$ of the coupling constant $e$ and a variable $y$ such that at $e^{2} \rightarrow 0$ the leading asymptotic is given by $F(y, e)=Q(e) f_{0}(y)+\cdots$, we call $f_{0}(y)$ a leading term and denote it by $[F(e, y)]_{0}$.

Consider first an asymptotic of an integral over $H$. We have the following identity:

$$
\left(\int D H \mathrm{e}^{\int_{D}\left(\frac{1}{\mathrm{e}^{2}} H F(A)+\mathcal{F}_{1}(H)\right.}\right)_{0}=\int d H_{0} \delta(F(A)) \mathrm{e}^{\int_{D} \mathcal{F}_{1}\left(H_{0}\right)},
$$

where $H_{0}$ is a constant mode of $H$ and $\mathcal{F}_{1}(H)$ is an arbitrary $\mathrm{e}^{2}$-independent function of $H$ such that the left-hand side of the identity is defined. Now let us find an asymptotic of the following integral:

$$
Z_{\mathcal{F}_{2}}=\int D b D \sigma \mathrm{e}^{\int_{D}\left(\frac{\imath}{\mathrm{e}^{2}} d b \wedge * d \sigma+b \mathcal{F}_{2}(\sigma)\right)}
$$

where $\mathcal{F}(\sigma)$ is a $\mathrm{e}^{2}$-independent function of $\sigma$. Consider a quadratic form

$$
\langle f, f\rangle=\int_{D} d f \wedge * d f
$$


on the space of functions such that $\left.\partial_{\theta} f\right|_{S^{1}=\partial D}=0$. We have the following obvious exact sequence:

$$
0 \rightarrow \operatorname{Fun}_{0}(D) \rightarrow \operatorname{Fun}(D) \rightarrow \operatorname{Fun}\left(S^{1}\right) \rightarrow 0,
$$

where $\operatorname{Fun}(D)$ is the space of functions on the disk, $\operatorname{Fun}_{0}(D)$ is the subspace of functions taking zero values on the boundary $S^{1}=\partial D$ and $\operatorname{Fun}\left(S^{1}\right)$ is a space of functions on the boundary. Then the orthogonal complement $\operatorname{Fun}_{0}^{\vee}(D)$ to the space $\operatorname{Fun}_{0}(D)$ with respect to the quadratic form (2.17) is given by the space of harmonic functions $\Delta f=0$. Due to the constraint $\left.\partial_{\theta} f\right|_{S^{1}=\partial D}=0$ the space $\operatorname{Fun}_{0}^{\vee}(D)$ consists of constant functions. Taking into account that the quadratic form (2.17) is non-degenerate on $\operatorname{Fun}_{0}(D)$, we infer that the following asymptotic for (2.16) holds

$$
\left(Z_{\mathcal{F}_{2}}\right)_{0}=\frac{1}{\operatorname{det}^{\prime}(d * d)} \int d b_{0} d \sigma_{0} \mathrm{e}^{\left.\int_{D} b_{0} \mathcal{F}_{2}\left(\sigma_{0}\right)\right)},
$$

where $b_{0}$ and $\sigma_{0}$ are constant modes of $b$ and $\sigma$.

By construction the functional integral with the action (2.11) is invariant with respect to the gauge group $\mathcal{G}_{0}$ defined by (2.14). We fix the gauge symmetry using a gauge-fixing condition $d * A=0$. In the case of abelian gauge group the introduction of Faddeev-Popov ghosts is not necessary. Integration over $H$ implies a constraint $F(A)=d A=0$. The gauge-fixing condition for the gauge group (2.14) implies that the residual gauge symmetry group is a subgroup of constant gauge transformations. Therefore, one can take $A=0$ and the effective action functional is given by

$$
\begin{aligned}
S_{\mathrm{eff}}= & \frac{1}{2 \pi} \int d^{2} z\left\{\frac{1}{\mathrm{e}^{2}}\left[(\lambda \wedge d \xi)_{z \bar{z}}+(\lambda \wedge * d \eta)_{z \bar{z}}\right]\right. \\
& +\sum_{j=1}^{\ell+1}\left[-\imath F_{\bar{z}}^{j}\left(\partial_{z} \bar{\varphi}^{j}\right)+\imath \bar{F}_{z}^{j}\left(\partial_{\bar{z}} \varphi^{j}\right)-\imath \psi_{\bar{z}}^{j} \lambda_{z} \bar{\varphi}^{j}+\imath \bar{\psi}_{z}^{j} \lambda_{\bar{z}} \varphi^{j}+\imath \psi_{\bar{z}}^{j}\left(\partial_{z} \bar{\chi}^{j}\right)\right. \\
& \left.-\imath \bar{\psi}_{z}^{j}\left(\partial_{\bar{z}} \chi^{j}\right)\right]+\frac{\imath H_{0}}{2}\left(\sum_{j=1}^{\ell+1}\left|\varphi^{j}\right|^{2}-r^{2}\right)-\frac{\imath \xi}{2} \sum_{j=1}^{\ell+1}\left(\varphi^{j} \bar{\chi}^{j}+\bar{\varphi}^{j} \chi^{j}\right) \\
& +\eta \sum_{j=1}^{\ell+1}\left(\varphi^{j} \bar{\chi}^{j}-\bar{\varphi}^{j} \chi^{j}\right)+b_{0} \sum_{j=1}^{\ell+1}\left[2 \chi^{j} \bar{\chi}^{j}+2 \imath\left(\sigma_{0}+\sigma_{j}\right)\left|\varphi^{j}\right|^{2}\right. \\
& \left.\left.+\hbar\left(\bar{\varphi}^{j} \iota_{v_{0}} d \varphi^{j}-\varphi^{j} \iota_{v_{0}} d \bar{\varphi}^{j}\right)\right]\right\} .
\end{aligned}
$$


The integration over $F$ and $\bar{F}$ gives the constraints

$$
\bar{\partial} \varphi^{j}=0, \quad \partial \bar{\varphi}^{j}=0
$$

The solutions of the constraints are given by holomorphic functions

$$
\varphi^{j}=\varphi^{j}(z), \quad \bar{\varphi}^{j}=\bar{\varphi}^{j}(\bar{z})
$$

From now on $\varphi^{j}$ will denote holomorphic functions. Now consider an asymptotic of the integrals over odd variables

$$
\tilde{Z}_{\mathcal{F}_{3}}=\int D \lambda D \xi D \eta \mathrm{e}^{\int_{D}\left(-\frac{1}{\mathrm{e}^{2}} \lambda \wedge d \xi-\frac{1}{\mathrm{e}^{2}} \lambda \wedge * d \eta+\lambda \mathcal{F}_{3}(\eta, \xi)\right)}
$$

where $\mathcal{F}_{3}(\eta, \xi)$ is independent of $e$. Let us combine two zero forms $\xi$ and $\eta$ into a one form

$$
\rho=d \xi+* d \eta
$$

Taking integral over $\lambda$ in (2.19) we obtain the following asymptotic in the limit $\mathrm{e}^{2} \rightarrow 0$ :

$$
\left(\int D \lambda D \xi D \eta \mathrm{e}^{\int_{D}-\frac{1}{\mathrm{e}^{2}} \lambda \wedge d \xi-\frac{1}{\mathrm{e}^{2}} \lambda \wedge * d \eta+\lambda B}\right)_{0}=\operatorname{det}^{\prime}(d * d) \delta(\rho) .
$$

We have

$$
\partial_{z}(\xi+\imath \eta)=0, \quad \partial_{\bar{z}}(\xi-\imath \eta)=0
$$

and thus solutions of the equation $\rho=0$ are given by holomorphic functions $F(z)=\xi-\imath \eta$. This implies that the functions $\xi, \eta$ are harmonic. Taking into account the boundary conditions $\left.\partial_{\theta} \xi\right|_{S^{1}}=\left.\partial_{\theta} \eta\right|_{S^{1}}=0$, the functional integration over $\xi$ and $\eta$ reduces to the integration over constant modes $\xi_{0}$ and $\eta_{0}$.

Now integration over $\psi_{z}^{\bar{j}}$ and $\psi_{\bar{z}}^{j}$ provides constraints

$$
\partial_{\bar{z}} \chi^{j}=0, \quad \partial_{z} \bar{\chi}^{\bar{j}}=0,
$$

solved by holomorphic functions $\chi^{j}(z)$ :

$$
\chi^{j}=\chi^{j}(z), \quad \bar{\chi}^{j}=\overline{\chi^{j}(z)} .
$$

From now on $\chi^{j}$ will denote holomorphic functions. Combining all ingredients together we obtain the following functional integral:

$$
\int d \xi_{0} d \eta_{0} d H_{0} d b_{0} d \sigma_{0}\left[D^{2} \varphi^{j}\right]\left[D^{2} \chi^{j}\right] \mathrm{e}^{-S_{\text {eff }}}
$$


with

$$
\begin{aligned}
S_{\mathrm{eff}}= & \frac{\imath H_{0}}{4 \pi} \int_{0}^{2 \pi} d \theta\left(\sum_{j=1}^{\ell+1}\left|\varphi^{j}(\theta)\right|^{2}-r^{2}\right) \\
& -\frac{\imath \xi_{0}}{4 \pi} \int_{0}^{2 \pi} d \theta \sum_{j=1}^{\ell+1}\left(\bar{\varphi}^{j}(\theta) \chi^{j}(\theta)+\varphi^{j}(\theta) \bar{\chi}^{j}(\theta)\right) \\
& +\frac{\eta_{0}}{2 \pi} \int_{0}^{2 \pi} d \theta \sum_{j=1}^{\ell+1}\left(\varphi^{j}(\theta) \bar{\chi}^{j}(\theta)-\bar{\varphi}^{j}(\theta) \chi^{j}(\theta)\right) \\
& +2 b_{0} \sum_{j=1}^{\ell+1} \frac{1}{2 \pi} \int_{0}^{2 \pi} d \theta\left[\chi^{j}(\theta) \bar{\chi}^{j}(\theta)+\imath\left(\sigma_{0}+\sigma_{j}\right)\left|\varphi^{j}(\theta)\right|^{2}+\hbar \bar{\varphi}^{j}(\theta) \partial_{\theta} \varphi^{j}(\theta)\right],
\end{aligned}
$$

where $\varphi^{j}(\theta)$ and $\chi^{j}(\theta)$ are restrictions on $S^{1}=\partial D$ of even and odd holomorphic functions $\varphi^{j}(z), \chi^{j}(z)$. Here we make a change of variables

$$
\varphi_{n}^{j} \rightarrow \frac{\varphi_{n}^{j}}{n+1}, \quad \chi_{n}^{j} \rightarrow \frac{\chi_{n}^{j}}{n+1},
$$

in the expansions of $\varphi^{j}(z)$ and $\chi^{j}(z)$

$$
\varphi^{j}(z)=\sum_{n=0}^{\infty} \varphi_{n}^{j} z^{n}, \quad \chi^{j}(z)=\sum_{n=0}^{\infty} \chi_{n}^{j} z^{n} .
$$

This change of variables converts the integrals of the product of the holomorphic and antiholomorphic functions over the disk $D$ into integrals over the boundary $S^{1}$. By the standard properties of the canonical integration measure on super-manifold $\mathbb{R}^{N \mid N}$ the Jacobian of the change of variables $(2.22)$ is trivial. The resulting functional integral with the action (2.21) coincides with the one defined by (2.15).

Theorem 2.1. The functional integral in $S^{1} \times U_{\ell+1}$-equivariant topological sigma model with the deformed action (2.15) has the following finitedimensional integral representation:

$$
\begin{gathered}
(2 \pi \hbar)^{\frac{\ell-1}{2}}\left\langle\mathrm{e}^{\frac{r^{2}}{2} \mathcal{O}^{(0)}}\right\rangle=\frac{1}{2 \pi \hbar} \int_{\mathbb{R}-\imath \epsilon} d H_{0} \mathrm{e}^{\imath H_{0} \frac{r^{2}}{2}} \prod_{j=1}^{\ell+1} \Gamma_{1}\left(\imath H_{0}+\sigma_{j} \mid \hbar\right), \\
\mathcal{O}^{(0)}=\int_{S^{1}=\partial D} d \theta \sigma(\theta),
\end{gathered}
$$

where $\epsilon>\max \left(-\sigma_{j}\right), j=1, \ldots, \ell+1$. 
Proof. We prove (2.23) using the representation obtained in Proposition 2.1. We shall calculate the following functional integral:

$$
\frac{1}{(2 \pi)^{2}} \int d \xi_{0} d \eta_{0} d H_{0} d b_{0} d \sigma_{0}\left[D^{2} \varphi^{j}\right]\left[D^{2} \chi^{j}\right] \mathrm{e}^{-S_{\text {eff }}+\frac{r^{2}}{2} \sigma_{0}}
$$

Integrating over $b_{0}$ we obtain the delta-function factor

$$
\delta\left(\frac{1}{\pi} \int_{0}^{2 \pi} d \theta\left(\sum_{j=1}^{\ell+1} \imath \chi^{j}(\theta) \bar{\chi}^{j}(\theta)-\sum_{j=1}^{\ell+1}\left(\sigma_{0}+\sigma_{j}\right)\left|\varphi^{j}(\theta)\right|^{2}+\imath \hbar \bar{\varphi}^{j}(\theta) \partial_{\theta} \varphi^{j}(\theta)\right)\right) .
$$

Further integration over $\sigma_{0}$ gives

$$
\sigma_{0}(\varphi, \chi)=\frac{\frac{1}{2 \pi} \int_{0}^{2 \pi} d \theta\left(\sum_{j=1}^{\ell+1} \imath \chi^{j}(\theta) \bar{\chi}^{j}(\theta)-\sum_{j=1}^{\ell+1} \sigma_{j}\left|\varphi^{j}(\theta)\right|^{2}+\imath \hbar \bar{\varphi}^{j}(\theta) \partial_{\theta} \varphi^{j}(z)\right)}{\frac{1}{2 \pi} \int_{0}^{2 \pi} d \theta \sum_{j=1}^{\ell+1}\left|\varphi^{j}(\theta)\right|^{2}} .
$$

Taking into account that the integral over $H_{0}$ implies the constraint

$$
\frac{1}{2 \pi} \int_{0}^{2 \pi} d \theta \sum_{j=1}^{\ell+1}\left|\varphi^{j}(\theta)\right|^{2}=r^{2}
$$

we obtain

$\sigma_{0}(\varphi, \chi)=\frac{1}{2 \pi r^{2}} \int_{0}^{2 \pi} d \theta\left(\sum_{j=1}^{\ell+1} \imath \chi^{j}(\theta) \bar{\chi}^{j}(\theta)-\sum_{j=1}^{\ell+1} \sigma_{j}\left|\varphi^{j}(z)\right|^{2}+\imath \hbar \bar{\varphi}^{j}(\bar{z}) \partial_{\theta} \varphi^{j}(z)\right)$.

After integration over $H_{0}$ the integrand reduces to

$$
\begin{aligned}
& \frac{2 \pi}{2 r^{2}} \delta\left(-\frac{1}{4 \pi} \int_{0}^{2 \pi} d \theta\left(\sum_{j=1}^{\ell+1}\left|\varphi^{j}(\theta)\right|^{2}-r^{2}\right)\right) \\
& \quad \times \exp \left\{\left(\frac{\imath \xi_{0}}{2}+\eta_{0}\right) \frac{1}{2 \pi} \int_{0}^{2 \pi} d \theta \sum_{j=1}^{\ell+1} \bar{\varphi}^{j}(\theta) \chi^{j}(\theta)\right. \\
& \quad+\left(\frac{\imath \xi_{0}}{2}-\eta_{0}\right) \frac{1}{2 \pi} \int_{0}^{2 \pi} d \theta \sum_{j=1}^{\ell+1} \varphi^{j}(\theta) \bar{\chi}^{j}(\theta) \\
& \left.\quad+\frac{1}{4 \pi} \int_{0}^{2 \pi} d \theta\left(\sum_{j=1}^{\ell+1} \imath \chi^{j}(\theta) \bar{\chi}^{j}(\theta)-\sum_{j=1}^{\ell+1} \sigma_{j}\left|\varphi^{j}(\theta)\right|^{2}+\imath \hbar \bar{\varphi}^{j}(\theta) \partial_{\theta} \varphi^{j}(\theta)\right)\right\},
\end{aligned}
$$


where $\varphi(z)$ and $\chi(z)$ are even and odd holomorphic functions. We normalize the measure as follows:

$$
D^{2} \varphi D^{2} \chi=\prod_{j=1}^{\ell+1} \frac{\imath}{2} D \varphi^{j} D \bar{\varphi}^{j} \prod_{j=1}^{\ell+1} \frac{2}{\imath} D \chi^{j} D \bar{\chi}^{j} .
$$

In particular

$$
\int D^{2} \chi \exp \left\{\frac{\imath}{4 \pi} \int_{0}^{2 \pi} d \theta \chi^{j}(\theta) \bar{\chi}^{j}(\theta)\right\}=1 .
$$

It is useful to reintroduce the variable $H_{0}$ by writing the first delta-function in (2.25) in the integral form. Then integrating over odd variables $d \xi_{0}, d \eta_{0}$ and $d^{2} \chi$, we reduce the functional integral to the following one:

$$
\begin{aligned}
\left\langle\mathrm{e}^{\frac{r^{2}}{2} \mathcal{O}^{(0)}}\right\rangle= & \int_{\mathbb{R}-\imath \epsilon} d H_{0} D^{2} \varphi \mathrm{e}^{\frac{\imath r^{2} H_{0}}{2}} \exp \left(-\frac{1}{4 \pi} \int_{0}^{2 \pi} d \theta \sum_{j}\left(\left(\imath H_{0}+\sigma_{j}\right)\left|\varphi^{j}(\theta)\right|^{2}\right.\right. \\
& \left.\left.-\imath \hbar \bar{\varphi}^{j}(\theta) \partial_{\theta} \varphi^{j}(\theta)\right)\right),
\end{aligned}
$$

where $\epsilon>\max \left(-\sigma_{j}\right), j=1, \ldots, \ell+1$. We can rewrite the expression for correlator in equivalent form

$$
\left\langle\mathrm{e}^{\frac{r^{2}}{2} \mathcal{O}^{(0)}}\right\rangle=\int_{\mathbb{R}-\imath \epsilon} d H_{0} \mathrm{e}^{\frac{\imath r^{2} H_{0}}{2}} Z_{\mathrm{LSM}}\left(\imath H_{0}+\sigma_{j}, \hbar\right),
$$

where

$$
Z_{\mathrm{LSM}}\left(\sigma_{j}, \hbar\right)=\int\left[D^{2} \chi\right]\left[D^{2} \varphi\right] \mathrm{e}^{-S_{\mathrm{LSM}}\left(\sigma_{j}, \hbar\right)}
$$

is a functional integral with the action

$$
\begin{aligned}
S_{\mathrm{LSM}}\left(\sigma_{j}, \hbar\right)= & \frac{1}{4 \pi} \int_{0}^{2 \pi} d \theta\left(\sum_{j=1}^{\ell+1} \imath \chi^{j}(\theta) \bar{\chi}^{j}(\theta)-\sum_{j=1}^{\ell+1} \sigma_{j}\left|\varphi^{j}(\theta)\right|^{2}\right. \\
& \left.+\imath \hbar \bar{\varphi}^{j}(\theta) \partial_{\theta} \varphi^{j}(\theta)\right) .
\end{aligned}
$$

The functional integral (2.28) is a correlation function in $S^{1} \times U_{\ell+1^{-}}$ equivariant type- $A$ topological linear sigma model on $D$ with the target 
space $V=\mathbb{C}^{\ell+1}$. This functional integral was calculated in [17] with the following result:

$$
Z_{L S M}\left(\sigma_{j}, \hbar\right)=\prod_{j=1}^{\ell+1}(2 \pi \hbar)^{-\frac{1}{2}} \hbar^{\frac{\sigma_{j}}{\hbar}} \Gamma\left(\frac{\sigma_{j}}{\hbar}\right)=(2 \pi \hbar)^{-\frac{\ell+1}{2}} \prod_{j=1}^{\ell+1} \Gamma_{1}\left(\sigma_{j} \mid \hbar\right)
$$

provided $\operatorname{Re}\left(\imath H_{0}+\sigma_{j}\right)>0, j=1, \ldots, \ell+1$.

Using the expression (2.29) for $Z_{L S M}$ and noting that the integration contour $\left(\mathbb{R}-\imath \epsilon\right.$ for $\left.\epsilon>\max \left(-\sigma_{j}\right), j=1, \ldots, \ell+1\right)$ is compatible with the condition $\operatorname{Re}\left(\imath H_{0}+\sigma_{j}\right), j=1, \ldots, \ell+1$, we obtain the integral representation for $\left\langle\mathrm{e}^{\frac{r^{2}}{2} \mathcal{O}^{(0)}}\right\rangle$

$$
\left\langle\mathrm{e}^{\frac{r^{2}}{2} \mathcal{O}^{(0)}}\right\rangle=(2 \pi \hbar)^{-\frac{\ell+1}{2}} \int_{\mathbb{R}-\imath \epsilon} d H_{0} \mathrm{e}^{\imath H_{0} \frac{r^{2}}{2}} \prod_{j=1}^{\ell+1} \Gamma_{1}\left(\imath H_{0}+\sigma_{j} \mid \hbar\right) .
$$

This complete the proof of the theorem.

Corollary 2.1. The parabolic Whittaker function (1.21) associated with $\mathbb{P}^{\ell}$ has an infinite-dimensional integral representation as a correlation function in type-A topological $S^{1} \times U_{\ell+1}$-equivariant sigma model with the target space $\mathbb{P}^{\ell}$

$$
\Psi_{\lambda}(x)=(2 \pi \hbar)^{\frac{\ell-1}{2}}\left\langle\mathrm{e}^{\frac{r^{2}}{2} \mathcal{O}^{(0)}}\right\rangle
$$

where $\sigma_{j}=-\lambda_{j}$ and $x=-\hbar r^{2} / 2$.

\section{Mirror-dual type-B topological Landau-Ginzburg model}

In this section, we consider a type- $B$ topological Landau-Ginzburg model that is a mirror dual [33] to the topological type- $A$ sigma model with the target space $\mathbb{P}^{\ell}$ considered in the previous section. We calculate a correlation function in the Landau-Ginzburg model that is mirror dual to the correlation function (2.23). This calculation naturally provides another integral representation $(1.21)$ of $(1, \ell+1)$-Whittaker function associated with $\mathbb{P}^{\ell}$.

The mirror duals of the topological gauged linear sigma models were constructed in [24]. In the following, we apply this construction to a particular sigma model considered in the previous section. To provide a heuristic explanation of the construction let us recall that the topological gauge field multiplets can be obtained in a simple way from $\mathcal{N}=2$ SUSY gauge multiplets described by twisted chiral superfields. Under mirror symmetry 
twisted chiral superfields are transformed into chiral multiplets. Thus a mirror dual of the $U_{1}$-gauged type- $A$ topological linear sigma model with the target space $\mathbb{C}^{\ell+1}$ should be described by a mirror dual of a type- $A$ topological linear sigma model with the target space $\mathbb{C}^{\ell+1}$ interacting with an additional topologically twisted chiral multiplet $\Xi$. The mirror dual of the type- $A$ twisted linear sigma model was considered in [24] (see also [18]) and is described by a Landau-Ginzburg sigma model. A coupling with the additional topological multiplet $\Xi$ can be guessed from simple duality considerations in quadratic theories [24]. Actually we are interested in the dual to $S^{1} \times U_{\ell+1}$-equivariant $U_{1}$-gauged type- $A$ linear sigma-model. It is useful to replace $U_{\ell+1}$-equivariance by $U_{1}^{\ell+1}$-equivariance supplied with the overall invariance of the correlation functions with respect to the permutation group $S_{\ell+1}$ (the Weyl group of $U_{\ell+1}$ ). The resulting Landau-Ginzburg theory, dual to $S^{1} \times U_{1}^{\ell+1}$-equivariant $U_{1}$-gauged type- $A$ linear sigma-model, has the following superpotential:

$$
W(\phi, \sigma)=-\imath \hbar \sigma \frac{r^{2}}{2}+\sum_{j=1}^{\ell+1}\left(-\left(\imath \sigma+\sigma_{j}\right) \phi^{j}+\mathrm{e}^{\phi^{j}}\right)+\hbar \log (2 \pi \hbar),
$$

written in terms of the lowest components $\phi_{j}, j=1, \ldots,(\ell+1)$ and $\sigma$ of chiral superfields. In (3.1) $\sigma_{j}, j=1, \ldots, \ell+1$ are $U_{1}^{\ell+1}$-equivariant parameters and $x=\frac{r^{2}}{2}$ is a parameter of the Kähler structure of $\mathbb{P}^{\ell}$ in the dual-type$A$ model. Note that usually one considers the Landau-Ginzburg theory on $\left(\mathbb{C}^{*}\right)^{\ell+2}$ as a mirror dual to $U_{1}$-gauged linear sigma model associated with non-linear $\mathbb{P}^{\ell}$ sigma model (this implies in particular that one uses the variables $\Phi^{j}=e^{\phi_{j}}$ as the correct description of the target space of the theory). Taking into account that the mirror dual of $U_{\ell+1}$-equivariant $\mathbb{P}^{\ell}$ sigma model has superpotential (3.1) which is single-valued on the universal covering space $\mathbb{C}^{\ell+2}$ we use the coordinates $\phi_{j}$ below.

We would like to calculate a particular correlation function on a disk $D$ in the Landau-Ginzburg topological theory with the superpotential (3.1). Happily this calculation was already done in [18] for an arbitrary superpotential $W(\phi)$. Let us recall briefly this derivation. We consider a set of fields with the following $S^{1}$-equivariant BRST transformations:

$$
\begin{aligned}
& \delta_{S^{1}} \phi_{-}^{i}=\eta^{i}, \quad \delta_{S^{1}} \eta^{i}=-\hbar \iota_{v_{0}} d \phi_{-}^{i}, \quad \delta_{S^{1}} \theta^{i}=G_{-}^{i}, \quad \delta_{S^{1}} G_{-}^{i}=-\hbar \iota_{v_{0}} d \theta^{i} \\
& \delta_{S^{1}} \rho^{i}=-d \phi_{+}^{i}+\hbar \iota_{v_{0}} G_{+}^{i}, \quad \delta_{S^{1}} \phi_{+}^{i}=-\hbar \iota_{v_{0}} \rho^{i}, \quad \delta_{S^{1}} G_{+}^{i}=d \rho^{i},
\end{aligned}
$$

where $\phi_{+}$and $\phi_{-}$are even real zero-form-valued fields, $\eta^{i}$ and $\theta^{i}$ are odd real zero-form-valued fields, $\rho^{i}$ are odd real one-form-valued fields, $G_{-}^{i}$ are even 
real zero-form-valued fields and $G_{+}^{i}$ are even real two-form-valued fields. The action of the topological sigma model is given by

$$
\begin{aligned}
S= & -\imath \sum_{j=1}^{N} \int_{D}\left(\left(d \phi_{+}^{j}-\hbar \iota_{v_{0}} G_{+}^{j}\right) \wedge * d \phi_{-}^{j}+\rho^{j} \wedge * d \eta^{j}-\theta_{j} d \rho^{j}+G_{+}^{j} G_{-}^{j}\right) \\
& +\sum_{i, j=1}^{N} \int_{D} d^{2} z \sqrt{h}\left(-\frac{\partial^{2} W_{-}\left(\phi_{-}\right)}{\partial \phi_{-}^{i} \partial \phi_{-}^{j}} \eta^{i} \theta^{j}-\imath \frac{\partial W_{-}\left(\phi_{-}\right)}{\partial \phi_{-}^{i}} G_{-}^{i}\right) \\
& +\sum_{i, j=1}^{N} \int_{D}\left(-\frac{1}{2} \frac{\partial^{2} W_{+}\left(\phi_{+}\right)}{\partial \phi_{+}^{i} \partial \phi_{+}^{j}} \rho^{i} \wedge \rho^{j}+\frac{\partial W_{+}\left(\phi_{+}\right)}{\partial \phi_{+}^{i}} G_{+}^{i}\right) \\
& +\frac{1}{\hbar} \int_{S^{1}=\partial D} d \sigma W_{+}\left(\phi_{+}\right) .
\end{aligned}
$$

Here $W_{+}$and $W_{-}$are arbitrary independent regular functions on $\mathbb{R}^{N}$. Thus defined action is $\delta_{S^{1}}$-closed. Below we consider the case of $W_{-}\left(\phi_{-}\right)=0$ and $W_{+}\left(\phi_{+}\right)=W\left(\phi_{+}\right)$. Thus, we have

$$
\begin{aligned}
S= & -\imath \sum_{j=1}^{N} \int_{D}\left(\left(d \phi_{+}^{j}-\hbar \iota_{v_{0}} G_{+}^{j}\right) \wedge * d \phi_{-}^{j}+\rho^{j} \wedge * d \eta^{j}-\theta_{j} d \rho^{j}+G_{+}^{j} G_{-}^{j}\right) \\
& +\sum_{i, j=1}^{N} \int_{D}\left(-\frac{1}{2} \frac{\partial^{2} W\left(\phi_{+}\right)}{\partial \phi_{+}^{i} \partial \phi_{+}^{j}} \rho^{i} \wedge \rho^{j}+\frac{\partial W\left(\phi_{+}\right)}{\partial \phi_{+}^{i}} G_{+}^{i}\right) \\
& +\frac{1}{\hbar} \int_{S^{1}=\partial D} d \sigma W\left(\phi_{+}\right) .
\end{aligned}
$$

Given an observable $\mathcal{O}$ (i.e., a functional of the fields), its correlation function is defined as the following functional integral:

$$
\begin{aligned}
\langle\mathcal{O}\rangle_{W} & :=\int D \mu \mathcal{O} \mathrm{e}^{-S} \\
D \mu & =\prod_{i=1}^{N}\left[D \phi_{+}^{i}\right]\left[D \phi_{-}^{i}\right]\left[D \eta^{i}\right]\left[D \theta^{i}\right]\left[D \rho^{i}\right]\left[D G_{+}^{i}\right]\left[D G_{-}^{i}\right] .
\end{aligned}
$$

We consider a local $\delta_{S^{1}}$-invariant observable

$$
\mathcal{O}=\left.\prod_{i=1}^{N} \delta\left(\phi_{-}^{i}(z, \bar{z})\right) \eta^{i}(z, \bar{z})\right|_{z=0}
$$


inserted at the center of the disk $D$. The calculation of the integral (3.5) with the observable (3.6) was done in [18].

Theorem 3.1. A correlation function of the observable (3.6) in the type- $B$ topological $S^{1}$-equivariant linear sigma model (3.4) is given by

$$
\langle\mathcal{O}\rangle_{W}=\int_{\mathbb{R}^{N}} \prod_{j=1}^{N} d t^{j} \mathrm{e}^{-\frac{1}{\hbar} W(t)}
$$

where $t^{j}$ are the constant modes of the fields $\phi_{+}^{j}$.

Now we can apply this general result to a particular case of the superpotential (3.1).

Proposition 3.1. The correlation function of the observable (3.6) in the type-B topological $S^{1}$-equivariant linear sigma model (3.4) with the superpotential

$$
W\left(\phi_{+}, \sigma\right)=-\imath \hbar \sigma \frac{r^{2}}{2}+\sum_{j=1}^{\ell+1}\left(-\left(\imath \sigma+\sigma_{j}\right) \phi_{+}^{j}+\mathrm{e}^{\phi_{+}^{j}}\right)+\hbar \log (2 \pi \hbar),
$$

is given by

$$
\begin{aligned}
\langle\mathcal{O}\rangle_{W}= & \frac{1}{2 \pi \hbar} \int_{\mathbb{R}-\imath \epsilon} d \sigma_{0} \mathrm{e}^{\imath \sigma_{0} \frac{r^{2}}{2}} \int_{\mathbb{R}^{\ell+1}} \prod_{j=1}^{\ell+1} d t^{j} \mathrm{e}^{\frac{1}{\hbar} \sum_{j=1}^{\ell+1}\left(\left(\imath \sigma_{0}+\sigma_{j}\right) t^{j}-e^{t^{j}}\right)} \\
& \epsilon>-\sigma_{j}, j=1, \ldots, \ell+1
\end{aligned}
$$

where $\sigma_{0}$ and $t^{j}$ are the constant modes of the real fields $\sigma$ and $\phi_{+}^{j}$, respectively.

The expression (3.9) coincides with (2.23) and is equivalent to (1.21), (1.22) obtained in Section 1. Thus type- $A$ and type- $B$ topological sigma model representations related by mirror symmetry give rise to two different integral representations $(1.21),(1.22)$ of the same parabolic Whittaker function (1.24).

\section{Equivariant symplectic volume and its mirror}

In this section, following [18], we provide a simple heuristic derivation of the correlation function (2.23). This calculation directly relates type- $A$ and 
type- $B$ mirror-dual integral representations. First let us demonstrate that (1.22) can be understood as a limit of equivariant volumes of spaces of holomorphic maps of $\mathbb{P}^{1} \rightarrow \mathbb{P}^{\ell}$ when a degree of the maps goes to infinity. The compactified space of holomorphic maps $\mathbb{P}^{1} \rightarrow \mathbb{P}^{\ell}$ of a degree $d$ can be identified with $\mathbb{P}^{(\ell+1)(d+1)-1}$ as follows. Holomorphic maps of $\mathbb{P}^{1}$ into $\mathbb{P}^{\ell}$ of degree $d$ can be described as a collection of $\ell+1$ mutually prime polynomials of the degree $d$

$$
\varphi^{j}(z)=\sum_{m=0}^{d} \varphi_{m}^{j} z^{m}, \quad j=1, \ldots, \ell+1,
$$

modulo multiplication by rational functions

$$
\varphi^{j}(z) \longrightarrow \tilde{\varphi}^{j}(z)=g(z) \varphi^{j}(z)
$$

such that the resulting functions $\tilde{f}^{j}(z)$ are again polynomials of the degree $d$. In the case of mutually prime polynomials the function $g(z)$ is necessary constant and thus the space $\mathcal{M}_{d}\left(\mathbb{P}^{1}, \mathbb{P}^{\ell}\right)$ of holomorphic maps $\mathbb{P}^{1} \rightarrow \mathbb{P}^{\ell}$ is given by a projectivization of the space of mutually prime $\ell+1$ polynomials. This space is non-compact and its compactification $\mathcal{Q} \mathcal{M}_{d}\left(\mathbb{P}^{1}, \mathbb{P}^{\ell}\right)$ obtained by omitting condition to be mutually prime is a projectivization $\mathbb{P}^{(\ell+1)(d+1)-1}$ of the vector space of $(\ell+1)$-tuples polynomials of the degree d. The space $\mathcal{Q} \mathcal{M}_{d}\left(\mathbb{P}^{1}, \mathbb{P}^{\ell}\right)=\mathbb{P}^{(\ell+1)(d+1)-1}$ can be obtained as the Hamiltonian reduction of the space of $(\ell+1)$-tuples polynomials of the degree $d$ supplied with the symplectic structure

$$
\Omega=\frac{\imath}{2} \sum_{j=1}^{\ell+1} \sum_{m=0}^{d} \delta \varphi_{m}^{j} \wedge \delta \bar{\varphi}_{m}^{j}
$$

with respect to a diagonal action of $U_{1}$ generated by the vector field

$$
v_{U_{1}}=\imath \sum_{j=1}^{\ell+1} \sum_{m=0}^{d}\left(\varphi_{m}^{j} \frac{\delta}{\delta \varphi_{m}^{j}}-\bar{\varphi}_{m}^{j} \frac{\delta}{\delta \bar{\varphi}_{m}^{j}}\right) .
$$

The action of $U_{1}$ is Hamiltonian and the corresponding momentum (i.e., solution of the equation $\iota_{v_{U_{1}}} \Omega=\delta \mu$ ) is given by

$$
\mu=-\frac{1}{2} \sum_{j=1}^{\ell+1} \sum_{m=0}^{d}\left|\varphi_{m}^{j}\right|^{2}=-\frac{1}{4 \pi} \sum_{j=1}^{\ell+1} \int_{0}^{2 \pi} d \theta\left|\varphi^{j}(\theta)\right|^{2}
$$


Here $\varphi^{j}(\theta)$ denotes a restriction of the polynomial $\varphi^{j}(z)$ on the circle $z=\mathrm{e}^{\imath \theta}$, $\theta \in[0,2 \pi)$. The reduced space $\mathbb{P}^{(\ell+1)(d+1)-1}$ is naturally supplied with a symplectic structure $\Omega_{\text {ind }}$.

The symplectic space $\mathcal{Q} \mathcal{M}_{d}\left(\mathbb{P}^{1}, \mathbb{P}^{\ell}\right)$ allows a Hamiltonian action of the larger Lie group $S^{1} \times U_{1}^{\ell+1}$. In homogeneous coordinates it is given by

$$
\varphi_{m}^{j} \longrightarrow \varphi_{m}^{j} \mathrm{e}^{\imath \alpha_{j}} \mathrm{e}^{\imath m \beta}, \quad\left(\mathrm{e}^{\imath \beta}, \mathrm{e}^{\imath \alpha_{1}}, \ldots, \mathrm{e}^{\imath \alpha_{\ell+1}}\right) \in S^{1} \times U_{1} \times \cdots \times U_{1} .
$$

The action of the corresponding Lie algebra generators is realized by the vector fields

$$
\begin{aligned}
v_{S^{1}} & =\imath \sum_{j=1}^{\ell+1} \sum_{m=0}^{d} m\left(\varphi_{m}^{j} \frac{\delta}{\delta \varphi_{m}^{j}}-\bar{\varphi}_{m}^{j} \frac{\delta}{\delta \bar{\varphi}_{m}^{j}}\right), \\
v_{U(1)_{j}} & =\imath \sum_{m=0}^{d}\left(\varphi_{m}^{j} \frac{\delta}{\delta \varphi_{m}^{j}}-\bar{\varphi}_{m}^{j} \frac{\delta}{\delta \bar{\varphi}_{m}^{j}}\right), \quad j=1, \ldots, \ell+1 .
\end{aligned}
$$

The corresponding momenta are

$$
\begin{gathered}
\mu_{S^{1}}=-\frac{1}{2} \sum_{j=1}^{\ell+1} \sum_{m=0}^{d} m\left|\varphi_{m}^{j}\right|^{2}=-\frac{1}{4 \pi \imath} \sum_{j=1}^{\ell+1} \int_{0}^{2 \pi} d \theta \bar{\varphi}^{j}(\theta) \partial_{\theta} \varphi^{j}(\theta), \\
\mu_{j}=-\frac{1}{2} \sum_{m=0}^{d}\left|\varphi_{m}^{j}\right|^{2}=-\frac{1}{4 \pi} \int_{0}^{2 \pi} d \theta\left|\varphi^{j}(\theta)\right|^{2} .
\end{gathered}
$$

The $S^{1} \times U_{1}^{\ell+1}$-equivariant volume of $\mathcal{Q} \mathcal{M}_{d}\left(\mathbb{P}^{1}, \mathbb{P}^{\ell}\right)$ is defined as the following integral:

$$
Z_{d}=\int_{\mathcal{Q M}_{d}\left(\mathbb{P}^{1}, \mathbb{P} \mathbb{P}^{\ell}\right)} \mathrm{e}^{\Omega_{\mathrm{ind}}^{S^{1} \times U_{1}^{\ell+1}}}
$$

where $\Omega_{\text {ind }}^{S^{1} \times U_{1}^{\ell+1}}$ is an $S^{1} \times U_{1}^{\ell+1}$-equivariant extension of the symplectic structure $\Omega_{\text {ind }}$ on $\mathcal{Q} \mathcal{M}_{d}\left(\mathbb{P}^{1}, \mathbb{P}^{\ell}\right)$ induced from (4.2) by the Hamiltonian reduction. Let us identify $H_{S^{1} \times U_{1}^{\ell+1}}^{*}(\mathrm{pt})$ with $\mathbb{C}\left[\hbar, \sigma_{1}, \ldots, \sigma_{\ell+1}\right]$ where $\hbar$ corresponds to a generator of $S^{1}$ and $\sigma_{j}$ to a generator of $j$ th factor in $U_{1}^{\ell+1}$. 
Proposition 4.1. The equivariant volume (4.4) has the following integral representation:

$$
Z_{d}=\frac{1}{(2 \pi)^{2}} \int \frac{D^{2} \varphi D^{2} \chi D H_{0} D \xi_{0} D \eta_{0} D \lambda D \sigma_{0}}{\operatorname{Vol}\left(\mathrm{U}_{1}\right)} \mathrm{e}^{\frac{r^{2}}{2}} \phi \mathrm{e}^{-S}
$$

where

$$
\begin{aligned}
S= & \imath H_{0} \frac{1}{4 \pi} \int_{0}^{2 \pi} d \theta\left(\sum_{j=1}^{\ell+1}\left|\varphi^{j}(\theta)\right|^{2}-r^{2}\right)-\frac{\imath \xi_{0}}{4 \pi} \\
& \times \sum_{j=1}^{\ell+1} \int_{0}^{2 \pi} d \theta\left(\varphi^{j}(\theta) \bar{\chi}^{j}(\theta)+\bar{\varphi}^{j}(\theta) \chi^{j}(\theta)\right) \\
& +\frac{\eta_{0}}{2 \pi} \sum_{j=1}^{\ell+1} \int_{0}^{2 \pi} d \theta\left(\varphi^{j}(\theta) \bar{\chi}^{j}(\theta)-\bar{\varphi}^{j}(\theta) \chi^{j}(\theta)\right)+2 \lambda \sum_{j=1}^{\ell+1} \frac{1}{2 \pi} \\
& \left.\times \int_{0}^{2 \pi}\left(\imath\left(\sigma_{0}+\sigma_{j}\right)\left|\varphi^{j}(\theta)\right|^{2}+\hbar \bar{\varphi}^{j}(\theta) \partial_{\theta} \varphi^{j}(\theta)\right)+\chi^{j}(\theta) \bar{\chi}^{j}(\theta)\right) d \theta,
\end{aligned}
$$

where $\xi_{0}, \eta_{0}$ and $\chi^{j}, j=1, \ldots,(\ell+1)$ are considered to be Grassmann variables. Also the functions $\varphi^{j}(\theta)$ and $\chi^{j}(\theta)$ are restricted to be degree $d$ polynomials of $z=e^{\imath \theta}$ with the integration measure given by

$$
D^{2} \varphi=\prod_{j=1}^{\ell+1} \prod_{m=0}^{d} \frac{\imath}{2} d \varphi_{m}^{j} \wedge d \bar{\varphi}_{m}^{j}, \quad D^{2} \chi=\prod_{j=1}^{\ell+1} \prod_{m=0}^{d} \frac{2}{\imath} d \chi_{m}^{j} \wedge d \bar{\chi}_{m}^{j}
$$

Proof. The proof reduces to application of the standard technique (see, e.g. [10]) and is given in Appendix F.

Proposition 4.2. The equivariant symplectic volume (4.4) has the following integral representation

$$
Z_{d}=(2 \pi)^{(\ell+1)(d+1)-2} \int_{\mathbb{R}-\imath \epsilon} d H_{0} \frac{\mathrm{e}^{\frac{\imath r^{2}}{2} H_{0}}}{\prod_{j=1}^{\ell+1} \prod_{m=0}^{d}\left(\imath H_{0}+\sigma_{j}+\hbar m\right)}
$$

where $\epsilon>\max \left(-\sigma_{j}\right), j=1, \ldots, \ell+1$. 
Proof. Using Proposition 4.1, we have

$$
\begin{aligned}
-S+\frac{r^{2}}{2} \sigma_{0}= & \frac{-\imath H_{0}}{2} \sum_{j=1}^{\ell+1}\left(\sum_{m=0}^{d} \varphi_{m}^{j} \bar{\varphi}_{m}^{j}-\frac{1}{2} r^{2}\right) \\
& +\frac{\imath \xi_{0}}{2} \sum_{j=1}^{\ell+1} \sum_{m=0}^{d}\left(\varphi_{m}^{j} \bar{\chi}_{m}^{j}+\bar{\varphi}_{m}^{j} \chi_{m}^{j}\right)-\eta_{0} \sum_{j=1}^{\ell+1} \sum_{m=0}^{d}\left(\varphi_{m}^{j} \bar{\chi}^{j}-\bar{\varphi}_{m}^{j} \chi_{m}^{j}\right) \\
& -2 \lambda\left(\imath \sum_{j=1}^{\ell+1} \sum_{m=0}^{d}\left(\sigma_{0}+\sigma_{j}+\hbar m\right)\left|\varphi_{m}^{j}\right|^{2}+\chi_{m}^{j} \bar{\chi}_{m}^{j}\right)+\frac{r^{2}}{2} \sigma_{0} .
\end{aligned}
$$

Applying Proposition F.1 in Appendix F to $\mathcal{Q} \mathcal{M}_{d}\left(\mathbb{P}^{1}, \mathbb{P}^{\ell}\right)=\mathbb{P}^{(\ell+1)(d+1)-1}$ with the action of $S^{1} \times U_{1}^{\ell+1}$, we obtain

$$
Z_{d}=(2 \pi)^{(\ell+1)(d+1)-2} \int_{\mathbb{R}-\imath \epsilon} d H_{0} \frac{\mathrm{e}^{\frac{\imath r^{2}}{2} H_{0}}}{\prod_{j=1}^{\ell+1} \prod_{m=0}^{d}\left(\imath H_{0}+\sigma_{j}+\hbar m\right)},
$$

where $\epsilon>\max \left(-\sigma_{j}\right), j=1, \ldots, \ell+1$.

Now let us consider the limit $d \rightarrow \infty$ of (4.5). We use $\zeta$-function regularization to define infinite products (see, e.g. [32] and the Appendix in [17]). More precisely, define logarithm of the regularized infinite product as a derivative of the zeta-function

$$
\ln \left[\prod_{n=0}^{\infty}(\rho n+\lambda)\right]_{\mathrm{reg}}:=-\left.\partial_{s} \zeta_{\rho}(s, \lambda)\right|_{s=0}
$$

where $\zeta_{\rho}(s, \lambda)$ is an analytic continuation of the infinite sum

$$
\zeta_{\rho}(s, \lambda)=\sum_{n=0}^{\infty} \frac{1}{(\rho n+\lambda)^{s}}, \quad-\pi<\arg (\rho n+\lambda) \leq \pi, \quad \operatorname{Re}(s)>1 .
$$

We have

$$
\zeta_{\rho}(0, \lambda)=\frac{1}{2}-\frac{\lambda}{\rho}, \quad \partial_{s} \zeta_{\rho}(0, \lambda)=-\left(\frac{1}{2}-\frac{\lambda}{\rho}\right) \ln \rho+\ln \frac{1}{\sqrt{2 \pi}} \Gamma\left(\frac{\lambda}{\rho}\right),
$$

and thus for the regularized infinite product we obtain

$$
\left[\prod_{n=0}^{\infty}(\rho n+\lambda)\right]_{\mathrm{reg}}=\rho^{1 / 2-\lambda / \rho} \frac{(2 \pi)^{1 / 2}}{\Gamma(\lambda / \rho)}
$$


Taking $\lambda=\imath H_{0}+\sigma_{j}$ and $\rho=\hbar$, we have

$$
\left[\frac{1}{\prod_{n=0}^{\infty}\left(\imath H_{0}+\sigma_{j}+\hbar n\right)}\right]_{\mathrm{reg}}=\frac{1}{\sqrt{2 \pi \hbar}} \hbar^{\left(\imath H_{0}+\sigma_{j}\right) / \hbar} \Gamma\left(\frac{\imath H_{0}+\sigma_{j}}{\hbar}\right)
$$

Now applying this regularization to the products in (4.5) for $d \rightarrow+\infty$, we obtain

$$
\begin{aligned}
\lim _{d \rightarrow \infty} \frac{(2 \pi \hbar)^{(\ell-1) / 2}}{(2 \pi)^{(\ell+1)(d+1)-2} Z_{d}=} & \frac{1}{2 \pi \hbar} \int_{\mathbb{R}-\imath \epsilon} d H_{0} \mathrm{e}^{\frac{\imath r^{2}}{2} H_{0}} \\
& \times \prod_{j=1}^{\ell+1} \hbar^{\left(\imath H_{0}+\sigma_{j}\right) / \hbar} \Gamma\left(\frac{\imath H_{0}+\sigma_{j}}{\hbar}\right)
\end{aligned}
$$

where we imply that $-\pi<\arg \left(\imath H+\sigma_{j}+\hbar n\right) \leq n \pi$ and $\epsilon>\max \left(-\sigma_{j}\right), j=$ $1, \ldots, \ell+1$. Thus taking in appropriate way the limit $d \rightarrow \infty$ we recover the integral representation (2.23) for the functional integral in topological field theory on the disk $D$. Note that the interpretation of the limit $d \rightarrow \infty$ as a replacement of $\mathbb{P}^{1}$ by $D$ seems quite natural as in the non-compact case there is no notion of a finite degree map.

To relate the type- $A$ model calculations given above with the integral representation (3.9) arising in type- $B$ model we follow the strategy used in [18], i.e., we calculate the equivariant volume of the holomorphic maps using the Duistermaat-Heckman formula [12]. We would like to calculate the integral over the infinite-dimensional projective space $\mathbb{P} \mathcal{M}\left(D, \mathbb{C}^{\ell+1}\right)$ assuming $\hbar>0$ and $\sigma_{j}>0, j=1, \ldots, \ell+1$. Let

$$
Z(\hbar, \sigma)=\int_{\mathbb{P} \mathcal{M}\left(D, \mathbb{C}^{\ell+1}\right)} \mathrm{e}^{\hbar \tilde{\mu}_{S^{1}}+\sum_{j=1}^{\ell+1} \sigma_{j} \tilde{\mu}_{j}+\Omega(x)}
$$

where $\Omega$ is a symplectic form on $\mathbb{P} \mathcal{M}\left(D, \mathbb{C}^{\ell+1}\right)$ defined by the Hamiltonian reduction with momentum $x, \tilde{\mu}_{S^{1}}$ is a momentum corresponding to the $S^{1}$ action on $\mathbb{P} \mathcal{M}\left(D, \mathbb{C}^{\ell+1}\right)$ and $\tilde{\mu}_{j}, j=1, \ldots, \ell+1$ are momentum corresponding to the action of $U_{1}^{\ell+1}$ on $\mathbb{P} \mathcal{M}\left(D, \mathbb{C}^{\ell+1}\right)$. Note that the integral in (4.8) is an infinite-dimensional one and thus requires a proper regularization. One can formally apply the Corollary F.1 Appendix E to rewrite the integral as 
follows

$$
\begin{aligned}
Z(\hbar, \sigma)= & \frac{1}{2 \pi} \int d x_{1} \cdots d x_{\ell+1} \delta\left(x-\sum_{j=1}^{\ell+1} x_{j}\right) \\
& \times \prod_{j=1}^{\ell+1} 2 \pi \int_{\mathbb{P} \mathcal{M}(D, \mathbb{C})} \mathrm{e}^{x_{j} \omega_{\mathbb{P}(D, \mathbb{C})}+\sigma_{j} \mu_{j}^{\mathbb{P} \mathcal{M}(D, \mathbb{C})}+\hbar \tilde{\mu}_{S^{1}}} .
\end{aligned}
$$

Thus the calculation reduces to the calculation of the following integral:

$$
\begin{aligned}
Z_{1}(\hbar, \sigma) & =2 \pi \int_{\mathbb{P} \mathcal{M}(D, \mathbb{C})} e^{\hbar \tilde{\mu}_{S^{1}}+\sigma_{j} \tilde{\mu}_{j}+x_{j} \omega_{\mathbb{P} \mathcal{M}(D, \mathbb{C})}} \\
& =2 \pi \mathrm{e}^{-\sigma_{j} x_{j}} \int_{\mathbb{P} \mathcal{M}(D, \mathbb{C})} e^{\hbar \tilde{\mu}_{S^{1}}+x_{j} \omega_{\mathbb{M} \mathcal{M}(D, \mathbb{C})}}
\end{aligned}
$$

This integral was already calculated in [18]. Below, for completeness, we recall the main steps of the calculation. To calculate the integral (4.10) we use an infinite-dimensional version of the Duistermaat-Heckman formula [12] (for a detailed introduction into the subject see e.g. [3]). Let $M$ be a $2 N$-dimensional symplectic manifold with the Hamiltonian action of $S^{1}$ having only isolated fixed points. Let $\mu$ be the corresponding momentum. The tangent space $T_{p_{k}} M$ to a fixed point $p_{k} \in M^{S^{1}}$ has a natural action of $S^{1}$. Let $v$ be a generator of $\operatorname{Lie}\left(S^{1}\right)$ and let $\hat{v}$ be its action on $T_{p_{k}} M$. Then the following identity holds:

$$
\int_{M} \mathrm{e}^{\hbar \mu+\omega}=\sum_{p_{k} \in M^{S^{1}}} \frac{\mathrm{e}^{\hbar \mu\left(p_{k}\right)}}{\operatorname{det}_{T_{p_{k}} M} \bar{v} / 2 \pi} .
$$

Let us formally apply (4.11) to the integral (4.10). A set of fixed points of $S^{1}$ acting on $\mathbb{P} \mathcal{M}(D, \mathbb{C})$ can be easily found using linear coordinates on $\mathcal{M}(D, \mathbb{C})$ (considered as homogeneous coordinates on $\mathbb{P} \mathcal{M}(D, \mathbb{C})$ ). Let $\varphi(z)$ be a holomorphic map of $D$ to $\mathbb{C}$. It represents an $S^{1}$-fixed point on $\mathbb{P} \mathcal{M}(D, \mathbb{C})$ if rotations by $S^{1}$ can be compensated by an action of $U_{1}$

$$
\mathrm{e}^{\imath \alpha(\beta)} \varphi\left(\mathrm{e}^{\imath \beta} z\right)=\varphi(z), \quad \beta \in[0,2 \pi]
$$

It is easy to see that solutions of (4.12) are enumerated by non-negative integers and are given by

$$
\varphi^{(n)}(z)=\varphi_{n} z^{n}, \quad \varphi_{n} \in \mathbb{C}^{*} \quad n \in \mathbb{Z}_{\geq 0} .
$$


The tangent space to $\mathcal{M}(D, \mathbb{C})$ at an $S^{1}$-fixed point $\varphi^{(n)}$ has natural linear coordinates $\varphi_{m} / \varphi_{n}, m \in \mathbb{Z}_{\geq 0}, m \neq n$ where coordinates $\varphi_{k}, k \in \mathbb{Z}_{\geq 0}$ are defined by the series expansion of $\varphi \in \mathcal{M}(D, \mathbb{C})$

$$
\varphi(z)=\sum_{k=0}^{\infty} \varphi_{k} z^{k}
$$

After identification of $\hbar$ in (4.10) with a generator of $\operatorname{Lie}\left(S^{1}\right)$ its action on the tangent space at the fixed point is given by a multiplication of each $\varphi_{m} / \varphi_{n}$ on $(m-n)$. Thus, to define an analog of the denominator in the right hand side of the Duistermaat-Heckman formula (4.11) one should provide a meaning to the infinite product $\prod_{m=0, m \neq n}^{\infty} \hbar(m-n) / 2 \pi$. We use a $\zeta$-function regularization

$$
\begin{gathered}
\ln \left[\prod_{m \in \mathbb{Z}_{\geq 0}, m \neq n} \frac{\hbar}{2 \pi}(m-n)\right]_{a} \\
\quad:=-\left.\frac{\partial}{\partial s}\left(\sum_{m=1}^{n} \frac{e^{-\imath \pi s}}{(a \hbar m / 2 \pi)^{s}}+\sum_{m=1}^{\infty} \frac{1}{(a \hbar m / 2 \pi)^{s}}\right)\right|_{s \rightarrow 0},
\end{gathered}
$$

where $a$ is a normalization multiplier. The introduction of $a$ is to take into account a multiplicative anomaly $\operatorname{det}(A B) \neq \operatorname{det} A \cdot \operatorname{det} B$ appearing for generic operators $A$ and $B$. We specify $a$ at the final step of the calculation of (4.10).

Lemma 4.1. The regularized product (4.14) is given by

$$
\frac{1}{\left[\prod_{m \in \mathbb{Z}_{\geq 0}, m \neq n} \hbar(m-n) / 2 \pi\right]_{a}}=(-1)^{n} \frac{(a \hbar / 2 \pi)^{-n}}{n !} \frac{\sqrt{a \hbar}}{2 \pi}
$$

Proof. Using the Riemann $\zeta$-function

$$
\zeta(s)=\sum_{n=1}^{\infty} \frac{1}{n^{s}}
$$

one can express the right-hand side of (4.14) as follows:

$$
\ln \left[\prod_{m \in \mathbb{Z}_{\geq 0}, m \neq n} \frac{\hbar}{2 \pi}(m-n)\right]_{a}=(\zeta(0)+n) \ln a \hbar / 2 \pi+\ln n !-\zeta^{\prime}(0)+\imath \pi n .
$$


Taking into account $\zeta(0)=-\frac{1}{2}$ and $\zeta(0)^{\prime}=-\frac{1}{2} \ln 2 \pi$ we obtain (4.15).

Let us now calculate the difference of the values of $S^{1}$-momentum map $\tilde{\mu}_{S^{1}}$ at two $S^{1}$-fixed points $\varphi^{(n)}, \varphi^{(0)} \in \mathbb{P} \mathcal{M}(D, \mathbb{C})$. Consider an embedded projective line $\mathbb{P}^{1} \subset \mathbb{P} \mathcal{M}(D, \mathbb{C})$, containing $\varphi^{(n)}$ and $\varphi^{(0)}$. Let us choose homogeneous coordinates $\left[z_{0}: z_{1}\right]$ on $\mathbb{P}^{1}$ such that $\varphi^{(0)}=[1: 0]$ and $\varphi^{(n)}=$ $[0: 1]$. The action of $S^{1}$ on $\mathbb{P} \mathcal{M}(D, \mathbb{C})$ descends to the embedded $\mathbb{P}^{1}$ via the vector field

$$
V=\imath n\left\{w \frac{\partial}{\partial w}-\bar{w} \frac{\partial}{\partial \bar{w}}\right\}, \quad w=z_{1} / z_{0} .
$$

The pull back of the symplectic form $\Omega(t)$ is given by

$$
\omega_{\mathbb{P}^{1}}=\imath t \frac{d w \wedge d \bar{w}}{\left(1+|w|^{2}\right)^{2}} .
$$

The action of the vector field (4.16) on $\mathbb{P}^{1}$ is the Hamiltonian one. Let $\mu_{S^{1}}^{(n)}$ be the corresponding momentum given by a restriction of the momentum $\tilde{\mu}_{S^{1}}$ for $S^{1}$-action $\mathbb{P} \mathcal{M}(D, \mathbb{C})$. From the definition of the momentum map we have

$$
\mu_{S^{1}}^{(n)}\left(\varphi^{(n)}\right)-\mu_{S^{1}}^{(n)}\left(\varphi^{(0)}\right)=\int_{[1: 0]}^{[0: 1]} d \mu_{S^{1}}^{(n)}=\int_{[1: 0]}^{[0: 1]} \iota_{V} \omega_{\mathbb{P}^{1}}
$$

A momentum defined as a solution of the equation $i_{V} \omega=d \mu$ is unique up an additive constant. To fix this constant we normalize the momentum $\tilde{\mu}_{S^{1}}(\varphi)$ so that $\mu_{S^{1}}\left(\varphi^{(0)}\right)=0$. Thus, we obtain the following:

$$
\mu_{S^{1}}^{(n)}\left(\varphi^{(n)}\right)=-n t \int_{[1: 0]}^{[0: 1]} \frac{w d \bar{w}+\bar{w} d w}{\left(1+|w|^{2}\right)^{2}}=n t\left[\frac{1}{\left(1+|w|^{2}\right)}\right]_{0}^{\infty}=-n t
$$

Substituting (4.18) and (4.15) into (4.11) for $M=\mathbb{P} \mathcal{M}(D, \mathbb{C})$, we obtain

$$
\begin{aligned}
2 \pi \int_{\mathbb{P} \mathcal{M}(D, \mathbb{C})} \mathrm{e}^{\hbar \tilde{\mu}_{S^{1}}+t \omega_{\mathbb{P} \mathcal{M}(D, \mathrm{C})}} & =2 \pi \sqrt{\frac{a \hbar}{(2 \pi)^{2}}} \sum_{n=0}^{\infty}(-1)^{n} \frac{\mathrm{e}^{-n \hbar \hbar}}{(a \hbar / 2 \pi)^{n} n !} \\
& =\sqrt{a \hbar} \exp \left\{-\frac{2 \pi}{a \hbar} \mathrm{e}^{-\hbar t}\right\},
\end{aligned}
$$

where the dependence on the normalization constant $a$ reflects an ambiguity of the regularized infinite-dimensional integral. 
Taking into account (4.9), the regularized $S^{1} \times U_{\ell+1}$-equivariant symplectic volume of the projectivization of the space of holomorphic maps of $D$ into $\mathbb{C}^{\ell+1}$ can be written as follows:

$$
\begin{aligned}
Z_{\mathrm{reg}}= & \frac{1}{2 \pi} \int_{0}^{\infty} \cdots \int_{0}^{\infty} d x_{1} \cdots d x_{\ell+1} \delta\left(x-\sum_{j=1}^{\ell+1} x_{j}\right) \\
& \times \prod_{j=1}^{\ell+1} 2 \pi \mathrm{e}^{-\sigma_{j} x_{j}} \int_{\mathbb{P} \mathcal{M}(D, \mathbb{C})} \mathrm{e}^{x_{j} \omega_{\mathbb{P}(D, C)}+\hbar \tilde{\mu}_{S 1}} \\
= & (a \hbar)^{\frac{\ell+1}{2}} \int_{\mathbb{R}-\imath \epsilon} \mathrm{e}^{\imath H_{0} x} \prod_{j=1}^{\ell+1} \int_{0}^{\infty} d x_{j} \mathrm{e}^{-\left(\imath H_{0}+\sigma_{j}\right) x_{j}-\frac{2 \pi}{a \hbar} \mathrm{e}^{-\hbar x_{j}}} .
\end{aligned}
$$

Changing the variables

$$
u_{j}=-\hbar x_{j}-\ln \left(\frac{a \hbar}{2 \pi}\right), \quad u=x+\frac{1}{\hbar} \ln \left(\frac{a \hbar}{2 \pi}\right),
$$

we obtain

$$
\begin{aligned}
Z_{\mathrm{reg}}= & \frac{1}{4 \pi^{2}}\left(\frac{a}{\hbar}\right)^{\frac{\ell+1}{2}}\left(\frac{a}{2 \pi}\right)^{\frac{1}{\hbar} \sum \sigma_{j}} \int_{\mathbb{R}-\imath \epsilon} d H_{0} \mathrm{e}^{\imath H_{0} u} \prod_{j=1}^{\ell+1} \hbar^{\frac{\imath H_{0}+\sigma_{j}}{\hbar}} \\
& \times \int_{-\infty}^{-\frac{1}{\hbar} \ln \left(\frac{a \hbar}{2 \pi}\right)} d u_{j} \mathrm{e}^{\frac{1}{\hbar}\left(\imath H_{0}+\sigma_{j}\right) u_{j}-\mathrm{e}^{u_{j}}} .
\end{aligned}
$$

To get rid of the renormalization ambiguity we take the limit $a \rightarrow 0$

$$
\begin{aligned}
Z(\sigma, \hbar) & =\lim _{a \rightarrow 0} \frac{2 \pi}{\hbar}\left(\frac{\hbar}{a}\right)^{\frac{\ell+1}{2}}\left(\frac{2 \pi}{a}\right)^{\frac{1}{\hbar} \sum \sigma_{j}} Z_{\text {reg }} \\
& =\frac{1}{2 \pi \hbar} \int_{\mathbb{R}-\imath \epsilon} d H_{0} \mathrm{e}^{\imath H_{0} u} \prod_{j=1}^{\ell+1} \Gamma_{1}\left(\imath H_{0}+\sigma_{j} \mid \hbar\right), \quad \epsilon>\max \left(-\sigma_{j}\right) .
\end{aligned}
$$

Integrating over $H_{0}$, we obtain precisely the mirror dual integral representation (1.21) for the Whittaker function associated with $\mathbb{P}^{\ell}$

$$
\int_{\mathcal{C}} \prod_{j=1}^{\ell} d t_{j} \exp \left(-\frac{1}{\hbar}\left(\sum_{j=1}^{\ell} \lambda_{j} t_{j}+\lambda_{\ell+1}\left(x-\sum_{j=1}^{\ell} t_{j}\right)+\sum_{j=1}^{\ell} \mathrm{e}^{t_{j}}+\mathrm{e}^{x-\sum_{j=1}^{\ell} t_{j}}\right)\right),
$$

where $\sigma_{j}=\lambda_{j}, x=-\hbar u$. 


\section{Conclusions}

To conclude we briefly outline some directions for future research. The most obvious one is a generalization to the case of an arbitrary partial flag manifold $G / P$. Recall that partial flag spaces allow a description in terms of the non-abelian Hamiltonian reduction of a symplectic vector spaces and thus the corresponding type- $A$ sigma models can be represented as gauged linear sigma models (of quiver type) with non-abelian gauge groups. For $G / P=G r(m, \ell+m)$ the corresponding parabolic $\mathfrak{g l}_{\ell+1}$-Whittaker function (1.8) has the following integral representation generalizing (1.22) (see Appendix D for $m=2, \ell=1)$ :

$$
\Psi_{\lambda_{1}, \ldots, \lambda_{j+1}}(x)=\int_{\mathcal{C}} d \gamma_{1}, \ldots, d \gamma_{m} \mathrm{e}^{-\frac{x}{\hbar}\left(\gamma_{1}+\gamma_{2}+\cdots+\gamma_{m}\right)} \frac{\prod_{j=1}^{m} \prod_{a=1}^{\ell+1} \Gamma_{1}\left(\gamma_{j}-\lambda_{a} \mid \hbar\right)}{\prod_{i, j=1, i \neq j}^{m} \Gamma_{1}\left(\gamma_{i}-\gamma_{j} \mid \hbar\right)} .
$$

A detailed discussion of the (topological) gauged linear sigma models describing holomorphic maps into $G r(m, \ell+m)$ can be found in [29,34]. For a mirror description of the corresponding gauged linear sigma models see [24]. We expect that $G r(m, \ell+m)$ analogs of the correlation functions of the topological quantum field theories considered in the previous sections are given by the integral expressions (5.1). Note also that the Givental-type integral representation for Whittaker functions associated with classical groups was constructed in [15]. This provides a Landau-Ginzburg model description of the mirror dual to a type- $A$ topological sigma models on the flag manifolds associated with the classical groups. We are going to explicitly derive this mirror duality following the line of this note elsewhere.

Another direction to pursue is a higher-dimensional generalizations of the constructions proposed in this note. One of the motivations is a higherdimensional generalization of the connection between Archimedean Langlands correspondence and mirror symmetry. This includes in particular an instanton counting in higher dimensions. Note also that the higherdimensional examples considered in [17] provide also additional insights into the conjectured relation between local Archimedean Langlands correspondence and the mirror symmetry in two dimensions. We are going to discuss various higher-dimensional generalization of the results of $[17,18]$ and of this note elsewhere. 


\section{Acknowledgments}

The research was supported by Grant RFBR-09-01-93108-NCNIL-a. The research of AG was also partly supported by Science Foundation Ireland grant and the research of SO was partially supported by Deligne's 2004 Balzan prize in Mathematics. The authors are thankful to Max-PlanckInstitut für Mathematik in Bonn for hospitality and excellent working conditions.

\section{Appendix A: A proof of Proposition 1.1}

We start with the following auxiliary result.

Lemma A.1. The adjoint action of the group element (1.9) on the algebra $\mathfrak{b}_{+}=\left\langle E_{i j}, i \leq j\right\rangle$ is given by the following:

$$
g^{-1} E_{11} g=E_{11}-\sum_{k=2}^{m} x_{k} E_{1, k}
$$

$g^{-1} E_{k k} g=E_{k k}+x_{k} E_{1, k}, \quad 2 \leq k \leq m$,

$g^{-1} E_{k, k+j} g=E_{k, k+j}+x_{k} E_{1, k+j}, \quad 2 \leq k \leq m-1, \quad 1 \leq j \leq m-k ;$

$$
\begin{gathered}
g^{-1} E_{m+k, m+k} g=E_{m+k, m+k}-x_{m+k} E_{m+k, \ell+m}, \quad 1 \leq k \leq \ell-1, \\
g^{-1} E_{m+k, m+k+j} g=E_{m+k, m+k+j}-x_{m+k+j} E_{m+k, \ell+m}
\end{gathered}
$$

$$
1 \leq k \leq \ell-2, \quad 1 \leq j \leq \ell-1-k
$$

$$
g^{-1} E_{\ell+m, \ell+m} g=E_{\ell+m, \ell+m}+\sum_{k=1}^{\ell-1} x_{m+k} E_{m+k, \ell+m} \text {. }
$$

(A.3) $g^{-1} E_{1, m+j} g=\mathrm{e}^{x_{1}-x_{\ell+m}}\left(E_{1, m+j}-x_{m+j} E_{1, \ell+m}\right), \quad 1 \leq j \leq \ell-1$,

$$
\begin{gathered}
g^{-1} E_{k, m+j} g=\mathrm{e}^{x_{1}-x_{\ell+m}}\left(E_{k, m+j}+x_{k} E_{1, m+j}-x_{m+j}\left(E_{k, \ell+m}+x_{k} E_{1, \ell+m}\right)\right), \\
2 \leq k \leq m, \quad 1 \leq j \leq \ell-1, \\
g^{-1} E_{k, \ell+m} g=\mathrm{e}^{x_{1}-x_{\ell+m}}\left(E_{k, \ell+m}+x_{k} E_{1, \ell+m}\right), \quad 2 \leq k \leq m, \\
g^{-1} E_{1, \ell+m} g=\mathrm{e}^{x_{1}-x_{\ell+m}} E_{1, \ell+m} .
\end{gathered}
$$

Proof. Consider functions $F_{i j}(x):=g(x)^{-1} E_{i j} g(x)$. The commutation relations above then can be derived by writing down and solving differential equations for $F_{i j}(x)$ with the initial condition $F_{i j}(0)=E_{i j}$. 
Let us introduce a notation

$$
\langle A\rangle:=\left\langle\psi_{\mathrm{L}}\left|\pi_{\underline{\lambda}}(A)\right| \psi_{\mathrm{R}}\right\rangle, \quad A \in \mathcal{U}_{\mathfrak{g l}_{\ell+m}} .
$$

Consider $\left\langle C_{2} g\right\rangle$ where $C_{2}$ is a quadratic Casimir operator (1.12). Taking into account $\left\langle\psi_{\mathrm{L}}\right| E_{j i}=0$ for $j-i>1$, we have $\left\langle C_{2} g\right\rangle=\left\langle\tilde{C}_{2} g\right\rangle$ with

$$
\tilde{C}_{2}=\sum_{i<j}^{\ell+m} E_{i i} E_{j j}-\sum_{j=1}^{\ell+m-1} E_{j+1, j} E_{j, j+1}-\sum_{i=1}^{\ell+m} \rho_{i} E_{i i}+\sigma_{2}(\rho) .
$$

Let us derive contributions of each term in (A.4). First, we observe that

$$
\begin{aligned}
& -\sum_{i=1}^{\ell+m} \rho_{i}\left\langle E_{i i} g\right\rangle=-\frac{\ell+m-1}{2}\left[\left\langle E_{11} g\right\rangle-\left\langle E_{\ell+m, \ell+m} g\right\rangle\right]-\sum_{j=2}^{\ell+m-1} \rho_{j}\left\langle E_{j j} g\right\rangle \\
& =-\frac{\ell+m-1}{2}\left[\left\langle\left(E_{11}+\cdots+E_{m m}\right) g\right\rangle\right. \\
& -\left\langle\left(E_{m+1, m+1}+\cdots+E_{\ell+m, \ell+m}\right) g\right\rangle-\sum_{k=2}^{m}\left\langle E_{k k} g\right\rangle \\
& \left.+\sum_{n=1}^{\ell-1}\left\langle E_{m+n, m+n} g\right\rangle\right]-\sum_{j=2}^{\ell+m-1} \rho_{j}\left\langle E_{j j} g\right\rangle \\
& =-\frac{\ell+m-1}{2}\left[\left\langle\left(E_{11}+\cdots+E_{m m}\right) g\right\rangle\right. \\
& \left.-\left\langle\left(E_{m+1, m+1}+\cdots+E_{\ell+m, \ell+m}\right) g\right\rangle\right] \\
& -\sum_{k=2}^{m}\left(\rho_{k}-\frac{\ell+m-1}{2}\right)\left\langle E_{k k} g\right\rangle \\
& -\sum_{n=1}^{\ell-1}\left(\rho_{m+n}+\frac{\ell+m-1}{2}\right)\left\langle E_{m+n, m+n} g\right\rangle \\
& =\frac{\ell+m-1}{2}\left[\frac{\partial}{\partial x_{1}}-\frac{\partial}{\partial x_{\ell+m}}\right] \\
& -\sum_{k=2}^{m}(k-1) x_{k} \frac{\partial}{\partial x_{k}}-\sum_{n=1}^{\ell-1}(\ell-n) x_{m+n} \frac{\partial}{\partial x_{m+n}},
\end{aligned}
$$

since $\rho_{k}-\rho_{1}=1-k$ with

$$
\left\langle E_{k k} g\right\rangle=\left\langle g\left(E_{k k}-x_{k} E_{1 k}\right)\right\rangle=-x_{k} \frac{\partial}{\partial x_{k}}\langle g\rangle, \quad k=2, \ldots, m,
$$


and $\rho_{m+n}-\rho_{\ell+m}=\ell-n$ with

$$
\begin{aligned}
\left\langle E_{m+n, m+n} g\right\rangle & =\left\langle g\left(E_{m+n, m+n}+x_{m+n} E_{m+n, \ell+m}\right)\right\rangle \\
& =x_{m+n} \frac{\partial}{\partial x_{m+n}}\langle g\rangle, \quad n=1, \ldots, \ell-1 .
\end{aligned}
$$

Next, by using $\left\langle\psi_{L}\right| E_{j+1, j}=\hbar^{-1}\left\langle\psi_{L}\right|$ for $j=1, \ldots, \ell+m-1$, we find the following:

$$
\begin{aligned}
-\sum_{j=1}^{\ell+m-1}\left\langle E_{j+1, j} E_{j, j+1} g\right\rangle= & -\frac{1}{\hbar} \sum_{j=1}^{\ell+m-1}\left\langle E_{j, j+1} g\right\rangle \\
= & -\frac{1}{\hbar}\left(\left\langle E_{12} g\right\rangle+\left\langle E_{\ell+m-1, \ell+m} g\right\rangle+\sum_{j=2}^{\ell+m-2}\left\langle E_{j, j+1} g\right\rangle\right) \\
= & \frac{1}{\hbar}\left\{\partial_{x_{2}}+\partial_{x_{\ell+m-1}}\right\}\langle g\rangle-\frac{1}{\hbar} \\
& \times \sum_{k=2}^{m-1}\left\langle g\left(E_{k, k+1}+x_{k} E_{1, k+1}\right)\right\rangle-\frac{1}{\hbar}\left\langle E_{m, m+1} g\right\rangle \\
& -\frac{1}{\hbar} \sum_{n=1}^{\ell-2}\left\langle g\left(E_{m+n, m+n+1}-x_{m+n+1} E_{m+n, \ell+m}\right)\right\rangle
\end{aligned}
$$

In generic case when $m>1, \ell>1$, we have

$$
\begin{aligned}
-\frac{1}{\hbar} & \left\langle E_{m, m+1} g\right\rangle=-\frac{1}{\hbar} \mathrm{e}^{x_{1}-x_{\ell+m}} \\
& \times\left\langle g\left(E_{m, m+1}+x_{m} E_{1, m+1}-x_{m+1} E_{m, \ell+m}-x_{m} x_{m+1} E_{1, \ell+m}\right)\right\rangle
\end{aligned}
$$

and therefore

(A.6)

$$
\begin{aligned}
-\frac{1}{\hbar} \sum_{j=1}^{\ell+m-1}\left\langle E_{j, j+1} g\right\rangle= & \frac{1}{\hbar}\left\{\frac{\partial}{\partial x_{2}}+\frac{\partial}{\partial x_{\ell+m-1}}+\sum_{k=2}^{m-1} x_{k} \frac{\partial}{\partial x_{k+1}}\right. \\
& \left.-\sum_{n=1}^{\ell-2} x_{m+n+1} \frac{\partial}{\partial x_{m+n}}+\frac{1}{\hbar}(-1)^{\epsilon(\ell, m)} x_{m} x_{m+1} \mathrm{e}^{x_{1}-x_{\ell+m}}\right\}\langle g\rangle .
\end{aligned}
$$


In the special case $m=1$, the following holds:

$$
\begin{aligned}
-\frac{1}{\hbar} \sum_{k=1}^{\ell}\left\langle E_{k, k+1} g\right\rangle & =-\frac{1}{\hbar} \sum_{k=1}^{\ell-1}\left\langle E_{k, k+1} g\right\rangle-\frac{1}{\hbar}\left\langle E_{\ell, \ell+1} g\right\rangle \\
& =-\frac{1}{\hbar} \sum_{k=1}^{\ell-1}\left\langle g E_{k, k+1}-x_{k+1} E_{k, \ell+1}\right\rangle-\frac{1}{\hbar}\left\langle E_{\ell, \ell+1} g\right\rangle \\
& =\left\{\frac{(-1)^{\epsilon(\ell, 1)}}{\hbar^{2}} x_{2} \mathrm{e}^{x_{\ell+1}-x_{1}}+\frac{1}{\hbar} \frac{\partial}{\partial x_{\ell}}-\frac{1}{\hbar} \sum_{j=2}^{\ell-1} x_{j+1} \frac{\partial}{\partial x_{j}}\right\}\langle g\rangle .
\end{aligned}
$$

In the other special case $\ell=1$, we have

$$
-\frac{1}{\hbar} \sum_{j=1}^{m}\left\langle E_{j, j+1} g\right\rangle=\frac{1}{\hbar}\left\{\frac{\partial}{\partial x_{2}}-\frac{(-1)^{\epsilon(1, m)}}{\hbar} x_{m} \mathrm{e}^{x_{m+1}-x_{1}}+\sum_{k=2}^{m-1} x_{k} \frac{\partial}{\partial x_{k+1}}\right\}\langle g\rangle .
$$

Finally, we calculate the contribution of the quadratic part of the Casimir element (A.4). We have

$$
\begin{aligned}
\sum_{i<j}\left\langle E_{i i} E_{j j} g\right\rangle= & \left\langle E_{11} E_{\ell+m, \ell+m} g\right\rangle+\sum_{k=2}^{m}\left\langle E_{11} E_{k k} g\right\rangle+\sum_{n=1}^{\ell-1}\left\langle E_{11} E_{m+n, m+n} g\right\rangle \\
& +\sum_{k=2}^{m}\left\langle E_{k k} E_{\ell+m, \ell+m} g\right\rangle+\sum_{n=1}^{\ell-1}\left\langle E_{m+n, m+n} E_{\ell+m, \ell+m} g\right\rangle \\
& +\sum_{2 \leq k<a}^{m}\left\langle E_{k k} E_{a a} g\right\rangle+\sum_{1 \leq n<b}^{\ell-1}\left\langle E_{m+n, m+n} E_{m+b, m+b} g\right\rangle \\
& +\sum_{k=2}^{m} \sum_{m=1}^{\ell-1}\left\langle E_{k k} E_{m+n, m+n} g\right\rangle .
\end{aligned}
$$


Then for each term in the above decomposition, we derive the following:

(A.10)

$$
\begin{aligned}
\left\langle E_{11} E_{\ell+m, \ell+m} g\right\rangle= & \left\langle\left(E_{11}+\cdots+E_{m m}\right)\left(E_{m+1, m+1}+\cdots+E_{\ell+m, \ell+m}\right) g\right\rangle \\
& -\sum_{k=2}^{m}\left\langle E_{k k} E_{\ell+m, \ell+m} g\right\rangle-\sum_{n=1}^{\ell-1}\left\langle\left(E_{11} E_{m+n, m+n} g\right\rangle\right. \\
& -\sum_{k=2}^{m} \sum_{n=1}^{\ell-1}\left\langle E_{k k} E_{m+n, m+n} g\right\rangle ;
\end{aligned}
$$

(A.11)

$$
\sum_{k=2}^{m}\left\langle E_{11} E_{k k} g\right\rangle=\sum_{k=2}^{m}\left\langle\left(E_{11}+\cdots+E_{m m}\right) E_{k k} g\right\rangle-\sum_{k, a=2}^{m}\left\langle E_{a a} E_{k k} g\right\rangle ;
$$

$$
\begin{aligned}
\sum_{n=1}^{\ell-1} & \left\langle E_{m+n, m+n} E_{\ell+m, \ell+m} g\right\rangle \\
= & \sum_{n=1}^{\ell-1}\left\langle E_{m+n, m+n}\left(E_{m+1, m+1}+\cdots+E_{\ell+m, \ell+m}\right) g\right\rangle \\
& \quad-\sum_{n, b=1}^{\ell-1}\left\langle E_{m+n, m+n} E_{m+b, m+b} g\right\rangle .
\end{aligned}
$$

Substituting (A.10)-(A.12) into (A.9), and making evident cancelations, we arrive at

$$
\begin{aligned}
\sum_{i<j}\left\langle E_{i i} E_{j j} g\right\rangle= & \left\langle\left(E_{11}+\cdots+E_{m m}\right)\left(E_{m+1, m+1}+\cdots+E_{\ell+m, \ell+m}\right) g\right\rangle \\
& -\sum_{k=2}^{m}\left\langle E_{k k}^{2} g\right\rangle-\sum_{n=1}^{\ell-1}\left\langle E_{m+n, m+n}^{2} g\right\rangle \\
& +\sum_{k=2}^{m}\left\langle\left(E_{11}+\cdots+E_{m m}\right) E_{k k} g\right\rangle \\
& -\sum_{k<a}^{m}\left\langle E_{k k} E_{a a} g\right\rangle-\sum_{n<b}^{m}\left\langle E_{m+n, m+n} E_{m+b, m+b} g\right\rangle
\end{aligned}
$$




$$
\begin{aligned}
& +\sum_{n=1}^{\ell-1}\left\langle E_{m+n, m+n}\left(E_{m+1, m+1}+\cdots+E_{\ell+m, \ell+m}\right) g\right\rangle \\
= & \left\{\frac{\partial^{2}}{\partial x_{1} \partial x_{\ell+m}}-\sum_{k=2}^{m}\left(x_{k}^{2} \frac{\partial^{2}}{\partial x_{k}^{2}}+x_{k} \frac{\partial}{\partial x_{k}}\right)\right. \\
& -\sum_{n=1}^{\ell-1}\left(x_{m+n}^{2} \frac{\partial^{2}}{\partial x_{m+n}^{2}}+x_{m+n} \frac{\partial}{\partial x_{m+n}}\right) \\
& -\sum_{2 \leq k<a}^{m} x_{k} x_{a} \frac{\partial^{2}}{\partial x_{k} \partial x_{a}}-\sum_{1 \leq n<b}^{\ell-1} x_{m+n} x_{m+b} \frac{\partial^{2}}{\partial x_{m+n} \partial x_{m+b}} \\
& \left.+\sum_{k=2}^{m} x_{k} \frac{\partial^{2}}{\partial x_{1} \partial x_{k}}-\sum_{n=1}^{\ell-1} x_{m+n} \frac{\partial^{2}}{\partial x_{m+n} \partial x_{\ell+m}}\right\}\langle g\rangle,
\end{aligned}
$$

since

$$
\begin{aligned}
\left\langle E_{k k}^{2} g\right\rangle & =\left(-x_{k} \partial_{x_{k}}\right)\left(-x_{k} \partial_{x_{k}}\right)\langle g\rangle=\left\{x_{k}^{2} \partial_{x_{k}}^{2}+x_{k} \partial_{x_{k}}\right\}\langle g\rangle, \quad 2 \leq k \leq m ; \\
\left\langle E_{m+n, m+n}^{2} g\right\rangle & =\left(x_{m+n} \partial_{x_{m+n}}\right)\left(x_{m+n} \partial_{x_{m+n}}\right)\langle g\rangle \\
& =\left\{x_{m+n}^{2} \partial_{x_{m+n}}^{2}+x_{m+n} \partial_{x_{m+n}}\right\}\langle g\rangle, \quad 1 \leq n \leq \ell-1 .
\end{aligned}
$$

At last, we collect (A.5), (A.6), (A.13), multiply by $\hbar^{2}$ and conjugate them by $\mathrm{e}^{-\rho_{1}\left(x_{1}-x_{\ell+m}\right)}$. We obtain (1.13). This completes the proof of Proposition 1.1.

\section{Appendix B: A proof of Proposition 1.2}

The lower-triangular part of the Lax-operator $\mathcal{L}=\left\|\mathcal{L}_{i j}\right\|$ easily follows from (1.6):

$$
\mathcal{L}_{n+1, n}=1, \quad \mathcal{L}_{n+j, n}=0, \quad 2 \leq j \leq \ell+m-n,
$$

for $1 \leq n \leq \ell+m-1$.

The calculation of the upper-triangular part of $\mathcal{L}$ can be done using Lemma A.1. Namely, if $\mathcal{L}_{i j}$ is in diagonal $(m \times m)$-block the following holds:

$$
\begin{aligned}
\mathcal{L}_{1, k}\langle g\rangle & =\hbar\left\langle E_{1, k} g\right\rangle=-\hbar \partial_{x_{k}}\langle g\rangle, \quad 2 \leq k \leq m ; \\
\mathcal{L}_{k k}\langle g\rangle & =\hbar\left\langle E_{k k} g\right\rangle=\hbar\left\langle g\left(E_{k k}+x_{k} E_{1, k}\right)\right\rangle=-\hbar x_{k} \partial_{x_{k}}\langle g\rangle, \quad 2 \leq k \leq m ;
\end{aligned}
$$


(B.2)

$$
\begin{aligned}
\mathcal{L}_{11}\langle g\rangle & =\hbar\left\langle\left(E_{11}+\cdots+E_{m m}\right) g\right\rangle-\hbar \sum_{k=2}^{m}\left\langle E_{k k} g\right\rangle \\
& =\left\{-\hbar \partial_{x_{1}}+\hbar \sum_{k=2}^{m} x_{k} \partial_{x_{k}}\right\}\langle g\rangle ; \\
\mathcal{L}_{k, k+j}\langle g\rangle & =\hbar\left\langle E_{k, k+j} g\right\rangle=\hbar\left\langle g\left(E_{k, k+j}+x_{k} E_{1, k+j}\right)\right\rangle \\
& =-\hbar x_{k} \partial_{x_{k+j}} 2 \leq k \leq m, \quad 1 \leq j \leq m-k .
\end{aligned}
$$

For the diagonal $(\ell \times \ell)$-block, we have

$$
\begin{aligned}
\mathcal{L}_{m+k, \ell+m}\langle g\rangle & =\hbar\left\langle E_{m+k, \ell+m} g\right\rangle=-\hbar \partial_{x_{m+k}}\langle g\rangle, \quad 1 \leq k \leq \ell-1 ; \\
\mathcal{L}_{m+k, m+k}\langle g\rangle & =\hbar\left\langle g\left(E_{m+k, m+k}-x_{m+k} E_{m+k, \ell+m}\right)\right\rangle \\
& =\hbar x_{m+k} \partial_{x_{m+k}}\langle g\rangle, \quad 1 \leq k \leq \ell-1 ; \\
\mathcal{L}_{\ell+m, \ell+m}\langle g\rangle & =\hbar\left\langle\left(E_{m+1, m+1}+\cdots+E_{\ell+m, \ell+m}\right) g\right\rangle-\hbar \sum_{k=1}^{\ell-1}\left\langle E_{m+k, m+k} g\right\rangle \\
& =-\hbar \partial_{x_{\ell+m}}\langle g\rangle-\hbar \sum_{k=1}^{\ell-1}\left\langle g\left(E_{m+k, m+k}-x_{m+k} E_{m+k, \ell+m}\right)\right\rangle \\
& =\left\{\hbar \partial_{x_{\ell+m}}-\hbar \sum_{k=1}^{\ell-1} x_{m+k} \partial_{x_{m+k}}\right\}\langle g\rangle ; \\
\mathcal{L}_{m+k, m+k+j}\langle g\rangle & =\hbar\left\langle g\left(E_{m+k, m+k+j}-x_{m+k+j} E_{m+k, \ell+m}\right)\right\rangle \\
& =\hbar x_{m+k+j} \partial_{x_{m+k}}\langle g\rangle, \\
1 & \leq k \leq \ell-2, \quad 1 \leq j \leq \ell-k-1 ;
\end{aligned}
$$

Finally, for the upper-triangular $(m \times \ell)$-block the following holds:

$$
\begin{aligned}
\mathcal{L}_{1, m+j}\langle g\rangle= & \hbar \mathrm{e}^{x_{1}-x_{\ell+m}}\left\langle g\left(E_{1, m+j}-x_{m+j} E_{1, \ell+m}\right)\right\rangle \\
= & -(-1)^{\epsilon(\ell, m)} x_{m+j} \mathrm{e}^{x_{1}-x_{\ell+m}}\langle g\rangle, \quad 1 \leq j \leq \ell-1 ; \\
\mathcal{L}_{1, \ell+m}\langle g\rangle= & \hbar \mathrm{e}^{x_{1}-x_{\ell+m}}\left\langle g E_{1, \ell+m}\right\rangle=(-1)^{\epsilon(\ell, m)} \mathrm{e}^{x_{1}-x_{\ell+m}}\langle g\rangle ; \\
\mathcal{L}_{k, \ell+m}\langle g\rangle= & \hbar \mathrm{e}^{x_{1}-x_{\ell+m}}\left\langle g\left(E_{k, \ell+m}+x_{k} E_{1, \ell+m}\right)\right\rangle \\
= & (-1)^{\epsilon(\ell, m)} x_{k} \mathrm{e}^{x_{1}-x_{\ell+m}}\langle g\rangle, \quad 2 \leq k \leq m ; \\
\mathcal{L}_{k, m+j}\langle g\rangle= & \hbar \mathrm{e}^{x_{1}-x_{\ell+m}}\left\langleg \left( E_{k, m+j}+x_{k} E_{1, m+j}\right.\right. \\
& \left.\left.-x_{m+j} E_{k, \ell+m}-x_{k} x_{m+k} E_{1, \ell+m}\right)\right\rangle \\
= & -(-1)^{\epsilon(\ell, m)} x_{k} x_{m+k} e^{x_{1}-x_{\ell+m}}\langle g\rangle, \quad 2 \leq k \leq m, \quad 1 \leq j \leq \ell-1 .
\end{aligned}
$$


After conjugation $\mathcal{L} \rightarrow \mathrm{e}^{-\rho_{1}\left(x_{1}-x_{\ell+m}\right)} \mathcal{L} \mathrm{e}^{\rho_{1}\left(x_{1}-x_{\ell+m}\right)}$, we arrive at the proof of Proposition 1.18.

\section{Appendix C: A proof of Theorem 1.1}

Consider the following decomposition of the Borel subalgebra $\mathfrak{b}_{+}=\mathfrak{h}^{(1, \ell+1)}+$ $\mathfrak{n}_{+}^{(1, \ell+1)}$ :

$$
\begin{aligned}
& \mathfrak{h}^{(1, \ell+1)}=\left\langle E_{11}, E_{11}+\cdots+E_{\ell+1, \ell+1}, E_{k, \ell+1}, 1<k \leq \ell\right\rangle, \\
& \mathfrak{n}_{+}^{(1, \ell+1)}=\left\langle E_{12}, E_{1, \ell+1} ; E_{k k}, 1<k \leq \ell ; E_{k, k+1}, 1<k<\ell\right\rangle .
\end{aligned}
$$

Recall the construction of a generalized Gelfand-Zetlin representation of $\mathfrak{g l}_{\ell+1}$ [13]. Namely, let $\underline{\gamma}_{1}, \ldots, \underline{\gamma}_{\ell+1}$ be a triangular array consisting of $\ell(\ell+$ 1) $/ 2$ variables $\gamma_{n}=\left(\gamma_{n 1}, \ldots, \gamma_{n n}\right) \in \mathbb{C}^{n}, n=1, \ldots, \ell+1$. The operators

$$
E_{k k}=\frac{1}{\hbar}\left(\sum_{j=1}^{n} \gamma_{n, j}-\sum_{i=1}^{n-1} \gamma_{n-1, i}\right), \quad 1 \leq k \leq \ell+1
$$

$$
\begin{aligned}
& E_{n, n+1}=-\frac{1}{\hbar} \sum_{i=1}^{n} \frac{\prod_{j=1}^{n+1}\left(\gamma_{n, i}-\gamma_{n+1, j}-\frac{\hbar}{2}\right)}{\prod_{s \neq i}\left(\gamma_{n, i}-\gamma_{n, s}\right)} \mathrm{e}^{-\hbar \partial_{n, i}}, \quad 1 \leq n \leq \ell ; \\
& E_{n+1, n}=\frac{1}{\hbar} \sum_{i=1}^{n} \frac{\prod_{j=1}^{n-1}\left(\gamma_{n, i}-\gamma_{n-1, j}+\frac{\hbar}{2}\right)}{\prod_{s \neq i}\left(\gamma_{n, i}-\gamma_{n, s}\right)} \mathrm{e}^{\hbar \partial_{n, i}}, \quad 1 \leq n \leq \ell,
\end{aligned}
$$

form a representation of $\mathfrak{g l}_{\ell+1}$ in the space $\mathcal{M}$ of meromorphic functions in $\ell(\ell+1) / 2$ variables $\left(\underline{\gamma}_{1}, \ldots, \underline{\gamma}_{\ell}\right)$. The Whittaker vectors $\left\langle\psi_{L}\right| \in \mathcal{V}_{\underline{\lambda}}^{\prime}$ and $\left|\psi_{\mathrm{R}}\right\rangle \in \mathcal{V}_{\underline{\lambda}}$ are defined by

$$
\left\langle\psi_{\mathrm{L}}\right| E_{n+1, n}=\frac{1}{\hbar}\left\langle\psi_{\mathrm{L}}\right|, \quad 1 \leq n \leq \ell ;
$$

$$
\begin{aligned}
& E_{12}\left|\psi_{\mathrm{R}}\right\rangle=0, \quad E_{1, \ell+1}\left|\psi_{\mathrm{R}}\right\rangle=\frac{(-1)^{1+\frac{\ell(\ell-1)}{2}}}{\hbar}\left|\psi_{\mathrm{R}}\right\rangle, \\
& E_{k k}\left|\psi_{\mathrm{R}}\right\rangle=0, \quad 2 \leq k \leq \ell, \quad E_{k, k+1}\left|\psi_{R}\right\rangle=0, \quad 2 \leq k \leq \ell-1 .
\end{aligned}
$$

We identify both $\mathcal{V}_{\underline{\lambda}}$ and $\mathcal{V}_{\underline{\lambda}}^{\prime}$ with subspaces of the space of functions of $\gamma_{i j}$, $i=1, \ldots, \ell+1, j=1, \ldots, i$. The action of $\mathcal{U}_{\mathfrak{g l}_{\ell+1}}$ on $\mathcal{V}_{\underline{\lambda}}$ is given by (C.2) and the action on $\mathcal{V}_{\underline{\lambda}}^{\prime}$ is given adjoint generators

$$
E_{i j}^{\dagger}=\mu^{-1}(\gamma) E_{i j} \mu(\gamma)
$$


where

$$
\mu(\gamma)=\prod_{n=2} \prod_{s \neq n} \Gamma^{-1}\left(\frac{\gamma_{n j}-\gamma_{n s}}{\hbar}\right)
$$

Lemma C.1. The equations (C.3) admit the solution

$$
\left\langle\left.\psi\right|_{\mathrm{L}}=\frac{1}{2 \pi \imath \hbar} ;\right.
$$

$$
\begin{aligned}
\left|\psi_{R}\right\rangle= & \prod_{k=2}^{\ell} \delta\left(\sum_{j=1}^{k} \gamma_{k, j}-\sum_{i=1}^{k-1} \gamma_{k-1, i}\right) \prod_{1 \leq i \leq k}^{\ell-1} \delta\left(\gamma_{k, i}-\gamma_{k+1, i}+\frac{\hbar}{2}\right) \\
& \times \prod_{n=2}^{\ell} \prod_{i_{n} \neq j_{n}} \Gamma\left(\frac{\gamma_{n, i_{n}}-\gamma_{n, j_{n}}}{\hbar}\right) \prod_{j=1}^{\ell+1} \hbar^{\frac{\gamma_{\ell, 1}-\gamma_{\ell+1, j}}{\hbar}+\frac{1}{2}} \Gamma\left(\frac{\gamma_{\ell, 1}-\gamma_{\ell+1, j}}{\hbar}+\frac{1}{2}\right)
\end{aligned}
$$

Proof. The equations on the left vector are similar to those in [13] and by the same reason admit the solution $\left\langle\left.\psi\right|_{L}=\frac{1}{2 \pi i \hbar}\right.$. To find the right Whittaker vector one needs an explicit expression of the element $E_{1, \ell+1}=\left[\ldots\left[\left[E_{12}\right.\right.\right.$, $\left.\left.\left.E_{23}\right], \ldots, E_{\ell-1, \ell}\right], E_{\ell, \ell+1}\right]$

$$
\begin{aligned}
E_{1, \ell+1}= & -\frac{1}{\hbar}\left(\sum_{i_{1}=1}^{\ell} \frac{\prod_{j_{1}=1}^{\ell+1}\left(\gamma_{\ell, i_{1}}-\gamma_{\ell+1, j_{1}}-\frac{\hbar}{2}\right)}{\prod_{k_{1} \neq i_{1}}\left(\gamma_{\ell, i_{1}}-\gamma_{\ell, k_{1}}\right)} \cdots\right. \\
& \left.\sum_{i_{\ell-1}=1}^{2} \frac{\prod_{j_{\ell-1} \neq i_{\ell-2}}\left(\gamma_{2, i_{2}}-\gamma_{3, j_{2}}-\frac{\hbar}{2}\right)}{\prod_{k_{\ell-1} \neq i_{\ell-1}}\left(\gamma_{2, i_{\ell-1}}-\gamma_{2, k_{\ell-1}}\right)} \prod_{j_{\ell} \neq i_{\ell-1}}\left(\gamma_{11}-\gamma_{2, j_{\ell}}-\frac{\hbar}{2}\right)\right) \\
& \times \mathrm{e}^{-\hbar \sum_{i_{1}=1}^{\ell} \sum_{i_{2}=1}^{\ell-1} \cdots \sum_{i_{\ell=1}}^{1} \sum_{k=1}^{\ell} \partial_{\gamma_{\ell+1-k, i_{k}}}}
\end{aligned}
$$

The constraints

$$
E_{22}\left|\psi_{\mathrm{R}}\right\rangle=\cdots=E_{\ell \ell}\left|\psi_{\mathrm{R}}\right\rangle=0,
$$

obviously hold. Similarly, due to the presence of the product of deltafunctions

$$
\prod_{1 \leq i \leq k}^{\ell-1} \delta\left(\gamma_{k, i}-\gamma_{k+1, i}+\frac{\hbar}{2}\right),
$$

we have $E_{12}\left|\psi_{R}\right\rangle=\cdots=E_{\ell-1, \ell}\left|\psi_{R}\right\rangle=0$. Thus we have to check that (C.6) satisfies the relation $E_{1, \ell+1}\left|\psi_{R}\right\rangle=\hbar^{-1}\left|\psi_{R}\right\rangle$ with (C.7). At first we note that 
due to the factor

$$
\prod_{1 \leq i \leq k}^{\ell-1} \delta\left(\gamma_{k, i}-\gamma_{k+1, i}+\frac{\hbar}{2}\right)
$$

we have

(C.8) $E_{1, \ell+1}\left|\psi_{R}\right\rangle$

$$
\begin{aligned}
= & -\frac{1}{\hbar} \frac{\prod_{j=1}^{\ell+1}\left(\gamma_{\ell, 1}-\gamma_{\ell+1, j}-\frac{\hbar}{2}\right)}{\prod_{k=2}^{\ell}\left(\gamma_{\ell, 1}-\gamma_{\ell, k}\right)} \cdots \frac{\left(\gamma_{21}-\gamma_{32}-\frac{\hbar}{2}\right)\left(\gamma_{21}-\gamma_{33}-\frac{\hbar}{2}\right)}{\gamma_{21}-\gamma_{22}} \\
& \times\left(\gamma_{11}-\gamma_{22}-\frac{\hbar}{2}\right) \mathrm{e}^{-\hbar \sum_{i_{1}=1}^{\ell} \sum_{i_{2}=1}^{\ell-1} \cdots \sum_{i_{\ell=1}}^{1} \sum_{k=1}^{\ell} \partial_{\gamma_{\ell+1-k}, i_{k}}}\left|\psi_{R}\right\rangle .
\end{aligned}
$$

Finally, straightforward calculations provide

$$
\begin{aligned}
E_{1, \ell+1}\left|\psi_{\mathrm{R}}\right\rangle & =(-1)^{1+\frac{\ell(\ell-1)}{2}} \prod_{k=1}^{\ell-1} \prod_{i=1}^{k} \frac{\gamma_{k, 1}-\gamma_{k+1, i+1}-\frac{\hbar}{2}}{\gamma_{k+1,1}-\gamma_{k+1, i+1}-\hbar}\left|\psi_{\mathrm{R}}\right\rangle \\
& =\frac{(-1)^{1+\frac{\ell(\ell-1)}{2}}}{\hbar}\left|\psi_{R}\right\rangle .
\end{aligned}
$$

Now we are ready to prove Proposition 1.1. Define the left and right $\mathcal{U}$-modules as $V^{\prime}=\left\langle\psi_{L}\right| \mathcal{U}$ and $V=\mathcal{U}\left|\psi_{R}\right\rangle$ respectively. Let $\phi \in V^{\prime}$ and $\psi \in V$. Define the paring $\langle\cdot, \cdot\rangle: V^{\prime} \otimes V \rightarrow \mathbb{C}$ by

$$
\langle\phi, \psi\rangle=\int_{\mathcal{C}} \mu(\gamma) \phi(\gamma) \psi(\gamma) \prod_{n=1}^{\ell} \prod_{j \leq n} d \gamma_{n j}
$$

where we define the integration domain $\mathcal{C}$ shortly below. Let $x=x_{1}$. We have

$$
\Psi_{\underline{\gamma}_{\ell+1}}^{(1, \ell+1)}(x, 0, \ldots, 0)=\mathrm{e}^{-\frac{\ell}{2} x}\left\langle\psi_{\mathrm{L}}, \mathrm{e}^{-x E_{11}} \psi_{\mathrm{R}}\right\rangle
$$

and thus

$$
\begin{aligned}
& \Psi_{\underline{\gamma}_{\ell+1}}^{(1, \ell+1)}(x, 0, \ldots, 0) \\
& \quad=\frac{1}{2 \pi \imath \hbar} \mathrm{e}^{-\frac{\ell}{2} x} \int_{\mathcal{C}_{n=1}} \prod_{\underline{\gamma}_{n}}^{\ell} \mu(\gamma) \mathrm{e}^{-\frac{1}{\hbar} x \gamma_{11}} \prod_{k=2}^{\ell} \delta\left(\sum_{j=1}^{k} \gamma_{k, j}-\sum_{i=1}^{k-1} \gamma_{k-1, i}\right)
\end{aligned}
$$




$$
\begin{aligned}
& \times \prod_{1 \leq i \leq k}^{\ell-1} \delta\left(\gamma_{k, i}-\gamma_{k+1, i}+\frac{\hbar}{2}\right) \prod_{n=2}^{\ell} \prod_{i_{n} \neq j_{n}} \Gamma\left(\frac{\gamma_{n, i_{n}}-\gamma_{n, j_{n}}}{\hbar}\right) \\
& \times \prod_{j=1}^{\ell+1} \hbar^{\frac{\gamma_{\ell, 1}-\gamma_{\ell+1, j}}{\hbar}+\frac{1}{2}} \Gamma\left(\frac{\gamma_{\ell, 1}-\gamma_{\ell+1, j}}{\hbar}+\frac{1}{2}\right),
\end{aligned}
$$

for appropriate choice of integration domain $\mathcal{C}$. Taking into account the Stirling formula for gamma function

$$
\Gamma(c+z)=\sqrt{2 \pi} z^{c+z-1 / 2} \mathrm{e}^{-z}(1+O(1 / z)
$$

for $z \rightarrow \infty, 0<|\arg (z)|<\pi$ and $c, z \in \mathbb{C}$ we infer that the integral (C.11) converges absolutely. Making obvious cancelations and integrating out the delta-functions one obtains

$$
\begin{aligned}
\Psi_{\underline{\gamma}_{\ell+1}}^{(1, \ell+1)}(x)= & \frac{1}{2 \pi \imath \hbar} \mathrm{e}^{-\frac{\ell}{2} x} \int_{\imath \mathbb{R}+\epsilon-\frac{\hbar}{2}} d \gamma_{\ell, 1} \mathrm{e}^{-\frac{1}{\hbar} \gamma_{\ell, 1} x+\frac{\ell-1}{2} x} \\
& \times \prod_{j=1}^{\ell+1} \hbar^{\frac{\gamma_{\ell, 1}-\gamma_{\ell+1, j}}{\hbar}+\frac{1}{2}} \Gamma\left(\frac{\gamma_{\ell, 1}-\gamma_{\ell+1, j}}{\hbar}+\frac{1}{2}\right),
\end{aligned}
$$

where $\epsilon>\gamma_{\ell+1, j}, j=1, \ldots, \ell+1$. Finally, introducing the variable $\gamma_{\ell, 1}=$ $\imath H-\frac{\hbar}{2}$ and setting $\lambda_{j}=\gamma_{\ell+1, j}$, we obtain (1.24).

\section{Appendix D: Explicit calculations for $\operatorname{Gr}(2,3)$}

In this appendix, we derive, using another version of the Gelfand-Zetlin realization, an integral representation for a specialization of the matrix elements (1.8) for $m=2, \ell=1$. Note that due to isomorphism $\operatorname{Gr}(1,3)=\operatorname{Gr}(2,3)=$ $\mathbb{P}^{2}$ the resulting integral expressions should be equal to (1.24) for $\ell=2$ after appropriate identification of the parameters. Below we explicitly verify this equivalence using an integral identity due to Gustafson [23].

We use the following version of the Gelfand-Zetlin realization of the universal enveloping algebra $\mathcal{U}\left(\mathfrak{g l}_{3}\right)$ (see [13]):

$$
\begin{aligned}
& E_{11}=\frac{1}{\hbar} \gamma_{11}, \quad E_{22}=\frac{1}{\hbar}\left(\gamma_{21}+\gamma_{22}-\gamma_{11}\right) \\
& E_{33}=\frac{1}{\hbar}\left(\gamma_{31}+\gamma_{32}+\gamma_{33}-\gamma_{21}-\gamma_{22}\right)
\end{aligned}
$$


(D.2)

$$
\begin{aligned}
& E_{21}=\frac{1}{\hbar} \mathrm{e}^{\hbar \partial_{11}} \\
& E_{32}=\frac{1}{\hbar}\left\{\frac{\gamma_{21}-\gamma_{11}+\frac{\hbar}{2}}{\gamma_{21}-\gamma_{22}} \mathrm{e}^{\hbar \partial_{21}}+\frac{\gamma_{22}-\gamma_{11}+\frac{\hbar}{2}}{\gamma_{22}-\gamma_{21}} \mathrm{e}^{\hbar \partial_{22}}\right\}
\end{aligned}
$$

(D.3)

$$
\begin{gathered}
E_{12}=-\frac{1}{\hbar}\left(\gamma_{11}-\gamma_{21}-\frac{\hbar}{2}\right)\left(\gamma_{11}-\gamma_{22}-\frac{\hbar}{2}\right) \mathrm{e}^{-\hbar \partial_{11}}, \\
E_{23}=-\frac{1}{\hbar}\left\{\frac{\prod_{j=1}^{3}\left(\gamma_{21}-\gamma_{3 j}-\frac{1}{2}\right)}{\gamma_{21}-\gamma_{22}} \mathrm{e}^{-\hbar \partial_{21}}-\frac{\prod_{j=1}^{3}\left(\gamma_{22}-\gamma_{3 j}-\frac{\hbar}{2}\right)}{\gamma_{22}-\gamma_{21}} \mathrm{e}^{-\hbar \partial_{22}}\right\}, \\
E_{13}=\left[E_{12}, E_{23}\right]=-\frac{1}{\hbar}\left\{\left(\gamma_{11}-\gamma_{22}-\frac{\hbar}{2}\right) \frac{\prod_{j=1}^{3}\left(\gamma_{21}-\gamma_{3 j}-\frac{\hbar}{2}\right)}{\gamma_{21}-\gamma_{22}} \mathrm{e}^{-\hbar \partial_{21}-\hbar \partial_{11}}\right. \\
\left.+\left(\gamma_{11}-\gamma_{21}-\frac{\hbar}{2}\right) \frac{\prod_{j=1}^{3}\left(\gamma_{22}-\gamma_{3 j}-\frac{\hbar}{2}\right)}{\gamma_{22}-\gamma_{21}} \mathrm{e}^{-\hbar \partial_{22}-\hbar \partial_{11}}\right\}
\end{gathered}
$$

The conjugated generators

$$
E_{i j}^{\dagger}=\mu^{-1}(\gamma) E_{i j} \mu(\gamma), \quad \mu(\gamma)=\frac{1}{\Gamma\left(\frac{\gamma_{21}-\gamma_{22}}{\hbar}\right) \Gamma\left(\frac{\gamma_{22}-\gamma_{21}}{\hbar}\right)}
$$

are given by

$$
\begin{aligned}
& E_{21}^{\dagger}=\frac{1}{\hbar} \mathrm{e}^{-\hbar \partial_{11}} \\
& E_{32}^{\dagger}=\frac{1}{\hbar}\left\{\frac{\gamma_{21}-\gamma_{11}-\frac{\hbar}{2}}{\gamma_{21}-\gamma_{22}} \mathrm{e}^{-\hbar \partial_{21}}+\frac{\gamma_{22}-\gamma_{11}-\frac{\hbar}{2}}{\gamma_{22}-\gamma_{21}} \mathrm{e}^{-\hbar \partial_{22}}\right\}
\end{aligned}
$$

In the case $\mathbb{P}^{2} \simeq G r(2,3)$ we have the following defining equations on the Whittaker vectors:

$$
\begin{array}{r}
\left\langle\psi_{\mathrm{L}}\right| E_{21}^{\dagger}=\frac{1}{\hbar}\left\langle\psi_{\mathrm{L}}\right|, \quad\left\langle\psi_{\mathrm{L}}\right| E_{32}^{\dagger}=\frac{1}{\hbar}\left\langle\psi_{\mathrm{L}}\right| ; \\
E_{22}\left|\psi_{\mathrm{R}}\right\rangle=0, \quad E_{13}\left|\psi_{\mathrm{R}}\right\rangle=-\frac{1}{\hbar}\left|\psi_{\mathrm{R}}\right\rangle, \quad E_{23}\left|\psi_{\mathrm{R}}\right\rangle=0 .
\end{array}
$$

The defining equations for the right vector (D.5) also can be solved. 
Lemma D.1. The Whittaker vectors defined by (D.5) read as follows:

$$
\left\langle\psi_{\mathrm{L}}\right|=\frac{1}{2 \pi \hbar}
$$

(D.7) $\left|\psi_{\mathrm{R}}\right\rangle=\prod_{k=1}^{2} \prod_{j=1}^{3} \hbar^{\left(\gamma_{2 i}-\gamma_{3 j}\right) / \hbar+1 / 2} \Gamma\left(\frac{\gamma_{2 i}-\gamma_{3 j}}{\hbar}+\frac{1}{2}\right) \delta\left(\gamma_{21}+\gamma_{22}-\gamma_{11}\right)$.

Proof. Direct verification.

Finally, we consider the $G r(2,3)$-Whittaker function

$$
\Psi_{\underline{\lambda}}^{(2,3)}\left(x_{1}, x_{2}, x_{3}\right)=\mathrm{e}^{-\left(x_{1}-x_{3}\right)}\left\langle\psi_{\mathrm{L}}\left|\pi_{\underline{\lambda}}\left(\mathrm{e}^{-x_{1}\left(E_{11}+E_{22}\right)-x_{2} E_{12}-x_{3} E_{33}}\right)\right| \psi_{\mathrm{R}}\right\rangle,
$$

with $\lambda_{j}=\gamma_{3, j}, j=1,2,3$ and $x_{1}=x, x_{2}=x_{3}=0$.

Proposition D.1. There is the following integral representation of $\operatorname{Gr}(2,3)$ Whittaker function:

$$
\begin{aligned}
\Psi_{\underline{\lambda}}^{(2,3)}(x)= & \frac{1}{2 \pi \hbar} \int_{\mathcal{C}_{\epsilon_{1}} \times \mathcal{C}_{\epsilon_{2}}} d \gamma_{21} d \gamma_{22} \mathrm{e}^{-\frac{1}{\hbar}\left(\sum_{j=1}^{2} \gamma_{2 j}\right) x} \\
& \times \frac{1}{\prod_{i \neq j} \Gamma_{1}\left(\gamma_{2 i}-\gamma_{2 j} \mid \hbar\right)} \prod_{j=1}^{3} \prod_{i=1}^{2} \Gamma_{1}\left(\gamma_{2 i}-\lambda_{j} \mid \hbar\right)
\end{aligned}
$$

where $\epsilon_{i}>\lambda_{j}, \quad i=1,2, \quad j=1,2,3$.

Proof. We have

$$
\begin{aligned}
\Psi_{\underline{\lambda}}^{(2,3)}(x)= & \frac{\mathrm{e}^{-x}}{2 \pi \hbar} \int d \gamma_{21} d \gamma_{22} d \gamma_{11} \delta\left(\gamma_{11}-\gamma_{21}-\gamma_{22}\right) \\
& \times \mathrm{e}^{\frac{1}{\hbar}\left(\sum \gamma_{3 j}-\sum \gamma_{2 k}\right) x} \frac{1}{\prod_{i \neq j} \Gamma\left(\frac{\gamma_{2 i}-\gamma_{2 j}}{\hbar}\right)} \\
& \times \prod_{j=1}^{3} \prod_{k=1}^{2} \hbar^{\left(\gamma_{2 k}-\lambda_{j}\right) / \hbar+1 / 2} \Gamma\left(\frac{\gamma_{2 k}-\lambda_{j}}{\hbar}+\frac{1}{2}\right)
\end{aligned}
$$


After taking integral over $\gamma_{11}$ and shifting the variables $\gamma_{21} \rightarrow \gamma_{21}-\hbar / 2$, $\gamma_{22} \rightarrow \gamma_{22}-\hbar / 2$ one obtains

(D.9) $\Psi_{\underline{\lambda}}^{(2,3)}(x)=\frac{1}{2 \pi \hbar} \int_{\mathcal{C}_{\epsilon_{1}} \times \mathcal{C}_{\epsilon_{2}}} d \gamma_{21} d \gamma_{22} \mathrm{e}^{-\frac{1}{\hbar}\left(\sum_{j=1}^{2} \gamma_{2 j}\right) x} \frac{1}{\prod_{i \neq j} \Gamma_{1}\left(\gamma_{2 i}-\gamma_{2 j} \mid \hbar\right)}$

$$
\times \prod_{j=1}^{3} \prod_{i=1}^{2} \Gamma_{1}\left(\gamma_{2 i}-\gamma_{3 j} \mid \hbar\right) \text {. }
$$

Proposition D.2. The following relation between (1,3)- and (2,3)Whittaker functions holds

$$
\Psi_{\lambda_{1}, \lambda_{2}, \lambda_{3}}^{(1,3)}(x)=\Psi_{\tilde{\lambda}_{1}, \tilde{\lambda}_{2}, \tilde{\lambda_{3}}}^{(2,3)}(x),
$$

where $\lambda_{i}=\tilde{\lambda}_{j}+\tilde{\lambda}_{k}, i \neq j \neq k$.

Proof. We have

$$
\begin{aligned}
\Psi_{\lambda_{1}, \lambda_{2}, \lambda_{3}}^{(2,3)}(x)= & \frac{1}{2 \pi \hbar} \int_{\mathcal{C}_{\epsilon_{1}} \times \mathcal{C}_{\epsilon_{2}}} d \gamma_{1} d \gamma_{2} \mathrm{e}^{-\frac{1}{\hbar}\left(\gamma_{1}+\gamma_{2}\right) x} \\
& \times \frac{\prod_{j=1}^{3} \Gamma_{1}\left(\gamma_{1}-\lambda_{j} \mid \hbar\right) \Gamma_{1}\left(\gamma_{2}-\lambda_{j} \mid \hbar\right)}{\Gamma_{1}\left(\gamma_{1}-\gamma_{2} \mid \hbar\right) \Gamma_{1}\left(\gamma_{2}-\gamma_{1} \mid \hbar\right)},
\end{aligned}
$$

where $\mathcal{C}_{\epsilon_{i}}=\imath \mathbb{R}+\epsilon_{i}, \epsilon_{i}>\lambda_{j}, j=1,2,3$. Let us introduce new variables $\gamma=\gamma_{1}+\gamma_{2}$ and $\gamma_{*}=\gamma_{1}-\gamma_{2}$ to obtain

$$
\begin{aligned}
\Psi_{\lambda_{1}, \lambda_{2}, \lambda_{3}}^{(2,3)}(x)= & \frac{1}{2 \pi \hbar} \int d \gamma d \gamma_{*} \mathrm{e}^{-\frac{1}{\hbar} \gamma x} \\
& \times \frac{\prod_{j=1}^{3} \Gamma_{1}\left(\frac{1}{2}\left(\gamma+\gamma_{*}\right)-\lambda_{j} \mid \hbar\right) \Gamma_{1}\left(\frac{1}{2}\left(\gamma-\gamma_{*}\right)-\lambda_{j} \mid \hbar\right)}{\Gamma_{1}\left(\gamma_{*} \mid \hbar\right) \Gamma\left(-\gamma_{*} \mid \hbar\right)} .
\end{aligned}
$$

Thus to establish equivalence with $\Psi^{(1,3)}$, we should prove

$$
\begin{aligned}
& \int_{\mathcal{C}_{\left(\epsilon_{1}-\epsilon_{2}\right) / 2}} d \gamma_{*} \frac{\prod_{j=1}^{3} \Gamma_{1}\left(\frac{1}{2}\left(\gamma+\gamma_{*}\right)-\lambda_{j} \mid \hbar\right) \Gamma_{1}\left(\frac{1}{2}\left(\gamma-\gamma_{*}\right)-\lambda_{j} \mid \hbar\right)}{\Gamma_{1}\left(\gamma_{*} \mid \hbar\right) \Gamma_{1}\left(-\gamma_{*} \mid \hbar\right)} \\
& \quad=\prod_{1 \leq i<j \leq 3} \Gamma_{1}\left(\gamma-\lambda_{i}-\lambda_{j} \mid \hbar\right) .
\end{aligned}
$$


This follows from the limiting form of the identity due to Gustafson [23] (Equation (9.4) with $n=1$ and $a_{4}=\infty$ )

$$
\int_{\mathcal{C}_{0}} d t \frac{\prod_{j=1}^{3} \Gamma_{1}\left(\alpha_{j}+t \mid \hbar\right) \Gamma_{1}\left(\alpha_{j}-t \mid \hbar\right)}{\Gamma_{1}(2 t \mid \hbar) \Gamma_{1}(-2 t \mid \hbar)}=\prod_{1 \leq i<j \leq 3} \Gamma_{1}\left(\alpha_{i}+\alpha_{j} \mid \hbar\right)
$$

where the integration contour $\mathcal{C}_{0}$ is goes between the sets of poles $\alpha_{i}+n \hbar$, $n \in \mathbb{Z}_{\geq 0}$ and $-\alpha_{i}-n \hbar, n \in \mathbb{Z}_{\geq 0}$.

\section{Appendix E: Gauge theory description of non-linear sigma models}

In this appendix, we recall the standard representation of a bosonic twodimensional sigma model with the target space $\mathbb{P}^{\ell}$ in terms of $U_{1}$-gauged sigma model. To simplify the arguments, we consider the equivalence of the classical theories, i.e., identifying the spaces of solutions of the equations of motions in two theories. Omitting fermionic fields in the action (2.9), we obtain the following action of the bosonic $U_{1}$-gauged sigma model

$$
\begin{aligned}
S_{\mathrm{bos}}= & \int_{\Sigma} d^{2} z \sqrt{h} h^{z \bar{z}} \\
& \times\left(\frac{1}{t}\left(\left(\partial_{\bar{z}}-A_{\bar{z}}\right) \varphi^{j}\right)\left(\partial_{z}-A_{z}\right) \bar{\varphi}^{j}+2 \imath b \sum_{j=1}^{\ell+1} \sigma\left|\varphi^{j}\right|^{2}+H\left(\sum_{j=1}^{\ell+1}\left|\varphi^{j}\right|^{2}-r^{2}\right)\right) .
\end{aligned}
$$

To obtain the classically equivalent field theory one may eliminate some fields using conditions of zero variations of the action $S_{\text {bos }}$ (substituting instead of an independent field $\Phi$ a solution of the equation $\delta S_{\text {bos }} / \delta \Phi=0$ ). Using a shift of the variable $H \rightarrow H-2 \imath b \sigma$ and eliminating the fields $b$ and $\sigma$ via zero variation condition, we obtain (up to some $r$-dependent additive constant)

$$
S_{\mathrm{bos}}=\int_{\Sigma} d^{2} z \sqrt{h} h^{z \bar{z}}\left(\frac{1}{t}\left(\left(\partial_{\bar{z}}-A_{\bar{z}}\right) \varphi^{j}\right)\left(\partial_{z}-A_{z}\right) \bar{\varphi}^{j}+H\left(\sum_{j=1}^{\ell+1}\left|\varphi^{j}\right|^{2}-r^{2}\right)\right) .
$$

Now it is easy to show that the field theory with the action (E.2) after a gauge fixing is equivalent on the classical level to the sigma model on $\mathbb{P}^{\ell}$ 
with the action

$$
S_{\sigma-\bmod }=\frac{1}{2} \int_{\Sigma} d^{2} z \sqrt{h} h^{z \bar{z}} G_{i \bar{j}}(\xi(z, \bar{z})) \partial_{\bar{z}} \xi^{i}(z, \bar{z}) \partial_{z} \bar{\xi}^{\bar{j}}(z, \bar{z}),
$$

where the Kähler metric $G(\xi)$ is associated with the Fubini-Studi two form on $\mathbb{P}^{\ell}$ locally written as

$$
\omega=\frac{\imath}{2 \pi t}\left(\sum_{j=1}^{\ell} \frac{d \xi_{j} \wedge d \bar{\xi}_{j}}{\left(1+\sum_{j=1}^{\ell}\left|\xi_{j}\right|^{2}\right)}-\frac{\left(\sum_{j=1}^{\ell} \bar{\xi}_{j} d \xi_{j}\right) \wedge\left(\sum_{i=1}^{\ell} \xi_{i} d \bar{\xi}_{i}\right)}{\left(1+\sum_{j=1}^{\ell}\left|\xi_{j}\right|^{2}\right)^{2}}\right) .
$$

Indeed, eliminating gauge fields $A$ by using zero variation condition we obtain

$$
\begin{aligned}
S_{\mathrm{bos}}= & \int_{\Sigma} d^{2} z \sqrt{h} h^{z \bar{z}} \\
& \times\left(\frac{1}{t}\left(\left(\partial_{\bar{z}}-A_{\bar{z}}(\varphi)\right) \varphi^{j}\right)\left(\partial_{z}-A_{z}(\varphi)\right) \bar{\varphi}^{j}+H\left(\sum_{j=1}^{\ell+1}\left|\varphi^{j}\right|^{2}-r^{2}\right)\right),
\end{aligned}
$$

with

$$
A_{\bar{z}}=\left(\sum_{j=1}^{\ell+1}\left|\varphi^{j}\right|^{2}\right)^{-1} \sum_{j=1}^{\ell+1} \bar{\varphi}^{j} \partial_{\bar{z}} \varphi^{j}, \quad A_{z}=\left(\sum_{j=1}^{\ell+1}\left|\varphi^{j}\right|^{2}\right)^{-1} \sum_{j=1}^{\ell+1} \varphi^{j} \partial_{z} \bar{\varphi}^{j} .
$$

Note that the action functional (E.4) is still invariant with respect to $U_{1}$ gauge symmetry

$$
\varphi^{i}(z) \longrightarrow \mathrm{e}^{\imath \alpha(z)} \varphi^{i}(z) .
$$

Zero variation condition over $H$ imposes the constraint

$$
\sum_{j=1}^{\ell+1}\left|\varphi^{j}\right|^{2}=r^{2}
$$

The solutions of (E.5) can be parameterized as follows:

$$
\begin{aligned}
\varphi^{j} & =\frac{\xi^{j}}{\left(r^{2}+\sum_{j=1}^{\ell}\left|\xi^{j}\right|^{2}\right)^{1 / 2}}, \quad j=1, \ldots, \ell, \\
\varphi_{\ell+1} & =\frac{r \mathrm{e}^{\imath \Theta}}{\left(r^{2}+\sum_{j=1}^{\ell}\left|\xi^{j}\right|^{2}\right)^{1 / 2}} .
\end{aligned}
$$


Fixing the gauge freedom by taking $\Theta=0$, we recover the standard representation (E.3) of $\mathbb{P}^{\ell}$ sigma model.

\section{Appendix F: Intersection theory on $\mathbb{P}^{\ell}$ via Hamiltonian reduction}

Let us given a manifold $X$ supplied with an action of a Lie group $G$ and an $G$-equivariant vector bundle $E$. Let $s$ be a section $E$ such that $G$ acts freely on the zero locus $s^{-1}(0)$. There is a universal representation of integrals of closed differential forms over factor $s^{-1}(0) / G$ in terms of integrals over $X$ (see, e.g. [10] for detailed exposition and relations with quantum field theory constructions). Below we review a simple instance of this construction providing a description of integrals of (equivariantly) closed differential forms over $\mathbb{P}^{\ell}$. In this case $X=\mathbb{C}^{\ell+1}$, the bundle $E$ is trivial and the group $G$ is abelian group $U_{1}$.

Let us supply complex vector space $\mathbb{C}^{\ell+1}$ with a symplectic structure

$$
\Omega=\frac{\imath}{2} \sum_{j=1}^{\ell+1} d \varphi^{j} \wedge d \bar{\varphi}^{j}
$$

The action of $U_{1}$

$$
\varphi^{j} \longrightarrow e^{\imath \alpha} \varphi^{j}, \quad \mathrm{e}^{\imath \alpha} \in U_{1},
$$

is Hamiltonian, i.e., there exists a momentum

$$
\mu(\varphi)=-\frac{1}{2} \sum_{j=1}^{\ell+1}\left|\varphi^{j}\right|^{2}
$$

such that $\iota_{v} \Omega=d \mu$ where

$$
v=\imath \sum_{j=1}^{\ell+1}\left(\varphi^{j} \frac{\partial}{\partial \varphi^{j}}-\bar{\varphi}^{j} \frac{\partial}{\partial \bar{\varphi}^{j}}\right)
$$

generates the action of $U_{1}$ on $\mathbb{C}^{\ell+1}$. Complex projective space $\mathbb{P}^{\ell}$ can be constructed via Hamiltonian reduction as a quotient of a hypersurface of the fixed level of the momentum $\mu$ over a free action of $U_{1}$

$$
\mathbb{P}^{\ell}=\mu^{-1}\left(\frac{1}{2} r^{2}\right) / U_{1}, \quad r \in \mathbb{R}
$$


Thus, constructed $\mathbb{P}^{\ell}$ is supplied with a canonical symplectic structure $\omega_{\mathbb{P} \ell}$ proportional to the Fubini-Study form. In terms of inhomogeneous coordinates $w_{j}=\varphi_{j} / \varphi_{\ell+1}, \varphi_{\ell+1} \neq 0$, it is given by

$$
\omega_{\mathbb{P}^{\ell}}=\frac{u r^{2}}{2} \frac{\left(1+\sum_{i=1}^{\ell}\left|w_{i}\right|^{2}\right) \sum_{j=1}^{\ell} d w_{j} \wedge d \bar{w}_{j}-\sum_{i, j}^{\ell} w_{i} \bar{w}_{j} d w_{j} \wedge d \bar{w}_{i}}{\left(1+\sum_{i=1}^{\ell}\left|w_{i}\right|^{2}\right)^{2}}
$$

The problem to write down the integral of closed differential forms over $\mathbb{P}^{\ell}$ in terms of integrals over $\mathbb{C}^{\ell+1}$ is naturally divided into two parts. First, we shall write an integral over hypersurface in a complex space in terms of an integral over the ambient space. Second, we shall write down an integral over a factor of a space over a free action of a Lie group in terms of an integral over a space before factorization. Let us first consider the problem of writing integral over hypersurface. Thus given a real valued function $s(x)$ on $\mathbb{R}^{N}$ let $i: Z \hookrightarrow \mathbb{R}^{N}$ be a zero locus subset of $s$ (we consider the case when $Z$ is compact). Let $R_{Z}$ be a de Rham current such that for a closed differential form $\omega$ on $\mathbb{R}^{N}$ the following holds

$$
\int_{Z} i^{*} \omega=\int_{\mathbb{R}^{N}} \omega \wedge R_{Z} .
$$

To write $R_{z}$ explicitly let us fix coordinates $\left(x^{1}, \ldots, x^{N}\right)$ on $\mathbb{R}^{N}$. We identify algebra of differential forms $\mathcal{A}^{*}\left(\mathbb{R}^{N}\right)$ on $\mathbb{R}^{N}$ with the algebra of functions $\operatorname{Fun}\left(\mathbb{R}^{N \mid N}\right)$ on the superspace $\mathbb{R}^{N \mid N}=\Pi T \mathbb{R}^{N}$ where $\Pi$ is a functor of the parity change of the fibers of vector bundles. Thus, we have associated coordinates $\left(x^{1}, \ldots, x^{N}, \psi^{1}, \ldots \psi^{N}\right)$ in $\mathbb{R}^{N \mid N}$ and de Rham differential is given by a vector field

$$
Q=\sum_{j=1}^{N} \psi^{i} \frac{\partial}{\partial x^{j}}, \quad Q^{2}=0 .
$$

Consider an extended space $\mathbb{R}^{N+1, N+1}=\mathbb{R}^{N \mid N} \times \mathbb{R}^{1 \mid 1}$ with the second factor understood as reversed-parity tangent bundle to the one-dimensional odd space $\mathbb{R}^{0 \mid 1}$. Let $(H, \xi)$ be coordinates in $\mathbb{R}^{1 \mid 1}$ and the de Rham differential on extended space is given by the vector field

$$
Q=\sum_{j=1}^{N} \psi^{j} \frac{\partial}{\partial x^{j}}+H \frac{\partial}{\partial \xi}
$$


Now a one-parameter family of the differential forms $R_{Z}(t)$ is given by the following Berezin integral over the superspace $\mathbb{R}^{1 \mid 1}$

$$
R_{Z}(t)=\frac{1}{2 \pi} \int_{\mathbb{R}^{1 \mid 1}} d \xi d H \exp \left(Q\left(\xi\left(\imath s(x)-\frac{t}{2} H\right)\right)\right)
$$

satisfies the relation (F.4) (see e.g. [10]).

To write down an integral of a closed differential form over a factor of a space $Y$ over a free action of Lie group $G=U_{1}$ in terms of an integral over $Y$ we use $G$-equivariant cohomology of $Y$. Let us recall that for a free action of a compact Lie group $G$ on $Y$ equivariant cohomology are isomorphic to the cohomology of the factor

$$
H_{G}^{*}(Y)=H^{*}(Y / G)
$$

Cartan model of $U_{1}$-equivariant de Rham complex on $X$ is given by (F.8)

$$
\Omega_{U_{1}}^{*}(Y)=\left(\Omega^{*}(Y)\right)^{U_{1}} \otimes \mathbb{C}[\sigma], \quad d_{G}=d-\iota_{v(\sigma)}, \quad \sigma \in \mathfrak{u}_{1}^{*}=\left(\operatorname{Lie}\left(U_{1}\right)\right)^{*},
$$

where $\sigma$ is of degree two. Equivariant cohomology $H_{U_{1}}^{*}(Y)$ is a module over the algebra $H_{U_{1}}^{*}(\mathrm{pt})=H^{*}\left(B U_{1}\right)$ isomorphic to $\mathbb{C}[\sigma]$. The algebra $H_{U_{1}}^{*}(\mathrm{pt})$ is generated by Chern class $c_{1}^{\text {univ }}$ of a universal $U_{1}$-bundle $E U_{1} \rightarrow B U_{1}$. Given a free action of $U_{1}$ on $Y$ one has a principle $U_{1}$-bundle $\mathcal{V}$ given by the projection $\pi: Y \rightarrow Y / U_{1}$. By definition of the universal bundle for a principle $U_{1}$-bundle $\mathcal{V}$ over $Y / U_{1}$ there exist a map $u: Y / U_{1} \rightarrow B U_{1}$ such that $\mathcal{V}$ is a pull back of the universal bundle over $B U_{1}$. and $c_{1}=u^{*}\left(c_{1}^{\text {univ }}\right)$ is a pull back of the first Chern class $c_{1}^{\text {univ }}$ of the universal bundle $E U_{1}$. The structure of $H_{U_{1}}^{*}(Y) \sim H^{*}\left(Y / U_{1}\right)$ as $H_{U_{1}}^{*}(\mathrm{pt})$-module is then defined by the condition that $c_{1}^{\text {univ }}$ acts on $H^{*}\left(Y / U_{1}\right)$ by a multiplication on $c_{1}$. In terms of the algebraic model (F.8) the image of the class $c_{1}^{\text {univ }}$ is represented by $\sigma$.

Now we would like to relate integration of cohomology classes in $H^{*}\left(Y / U_{1}\right)$ and $H_{U_{1}}^{*}(Y)$. Equivariant de Rham complex can be represented as a space of functions on a super-space $\Pi T Y \times \mathfrak{u}_{1}$ with an odd vector field $Q$ given in local coordinates $\left(y^{i}, \psi^{i}, \sigma\right)$ by

$$
Q=\sum_{i=1}^{M} \psi^{i} \frac{\partial}{\partial y^{i}}+\sigma v^{i}(y) \frac{\partial}{\partial \psi^{i}}
$$


To defined an integration over a $U_{1}$-factor we consider $S^{1}$-equivariant cohomology of the extended space $\tilde{Y}=Y \times \mathbb{R}^{0 \mid 1}$ where the last factor is understood as a reversed parity Lie algebra $\mathfrak{u}_{1}$. Then we have

$$
\Omega_{U_{1}}^{*}(\tilde{Y})=\left(\Omega^{*}(Y)\right)^{U_{1}} \otimes \mathbb{C}[\sigma] \otimes \mathbb{C}[\lambda, \eta],
$$

where the de Rham differential $Q_{U_{1}}$ acts as follows:

$$
Q_{U_{1}} y^{i}=\psi^{i}, \quad Q_{U_{1}} \psi^{i}=-\sigma v^{i}(y), \quad Q_{U_{1}} \lambda=\eta, \quad Q_{U_{1}} \eta=0 .
$$

Let $\omega(y, \psi, \sigma)$ be a representative of a cohomology class in $H_{U_{1}}^{*}(Y)$ and $\widetilde{\omega}$ is a representative of the corresponding class in $H^{*}\left(Y / U_{1}\right)$ according to identification (F.7). Then the following identity holds

$$
\text { (F.9) } \int_{Y / G} \widetilde{\omega}=\frac{1}{2 \pi} \int_{\Pi T Y \times \mathbb{R} \times \mathbb{R}^{1 \mid 1}} \frac{d y d \psi d \sigma d \lambda d \eta}{\operatorname{Vol}\left(U_{1}\right)} \omega(y, \psi, \sigma) \mathrm{e}^{\imath Q_{U_{1}}\left(\lambda v_{i}(y) \psi^{i}\right)},
$$

where $(\lambda, \eta)$ are local coordinates on $\mathbb{R}^{1 \mid 1}$. The identity (F.9) can be derived replacing $\omega(y, \psi, \sigma)$ by a representative of the same class in $H_{U_{1}}^{*}(Y)$ which does not contain $\sigma$. Then integrating over $\lambda, \eta$ and $\sigma$ in the right hand side of (F.9) one obtains the left hand side of (F.9).

Now we can apply (F.4) and (F.9) to the $\mathbb{P}^{\ell}$ obtained via Hamiltonian reduction of $\mathbb{C}^{\ell+1}$. Let us introduce the following variables:

$$
\left(\varphi^{i}, \bar{\varphi}^{i}, \chi^{i}, \bar{\chi}^{i}, \sigma\right), \quad(\xi, H), \quad(\lambda, \eta)
$$

and the action of the $U_{1}$-equivariant de Rham differential $Q_{U_{1}}$ is given by

$$
\begin{aligned}
Q_{U_{1}} \varphi^{i} & =\chi^{i}, \quad Q_{U_{1}} \chi^{i}=-\imath \sigma \varphi^{i}, \quad Q_{U_{1}} \xi=H, \quad Q_{U_{1}} H=0, \\
Q_{U_{1}} \lambda & =\eta, \quad Q_{U_{1}} \eta=0 .
\end{aligned}
$$

We take as a function $s(\varphi)$ on $X=\mathbb{C}^{\ell+1}$ the shifted momentum

$$
s(\varphi)=\mu(\varphi)+\frac{1}{2} r^{2} .
$$

Then we have

$$
\begin{aligned}
\int_{s^{-1}(0) / U_{1}} \widetilde{\omega}= & \int_{\Pi T Y \times \mathbb{R} \times \mathbb{R}^{2 \mid 2}} \frac{d^{2} \varphi d^{2} \chi d \sigma d \lambda d \xi d \eta d H}{(2 \pi)^{2} \operatorname{Vol}\left(\mathrm{U}_{1}\right)} \omega(\varphi, \bar{\varphi}, \chi, \bar{\chi}, \sigma) \\
& \times \mathrm{e}^{\imath Q_{U_{1}}\left(\lambda v_{i}(\varphi) \chi^{i}+\xi s(\varphi)\right)}
\end{aligned}
$$


where $\left.Y=s^{-1}(0), s^{-1}(0)\right) / U_{1}=\mathbb{P}^{\ell}$ according to (F.2) and we take $t=0$ in (F.4). We consider the following cohomology class:

$$
\widetilde{\omega}=\mathrm{e}^{a c_{1}} \in H^{*}\left(\mathbb{P}^{\ell}\right), \quad a \in \mathbb{R}
$$

where $c_{1}$ is a first Chern class of the line bundle $\mathcal{O}(1)$ on $\mathbb{P}^{\ell}$. As it was discussed above $c_{1}$ can be represented by $\sigma$ in Cartan model of equivariant cohomology. Thus we can chose $\omega=e^{a \sigma}$ as a representative of (F.12). Thus we have the following integral representation

$$
\begin{aligned}
\int_{\mathbb{P}^{\ell}} \mathrm{e}^{a c_{1}}= & \frac{1}{(2 \pi)^{2}} \int_{\Pi T Y \times \mathbb{R} \times \mathbb{R}^{2 \mid 2}} \frac{d^{2} \varphi d^{2} \chi d \sigma d \lambda d \xi d \eta d H}{\operatorname{Vol}\left(\mathrm{U}_{1}\right)} \mathrm{e}^{a \sigma} \\
& \times \exp \left(\imath H\left(-\frac{1}{2} \sum_{j=1}^{\ell+1} \varphi^{j} \bar{\varphi}^{j}+\frac{1}{2} r^{2}\right)+\frac{\imath \xi}{2} \sum_{i=1}^{\ell+1}\left(\varphi^{i} \bar{\chi}^{i}+\bar{\varphi}^{i} \chi^{i}\right)\right. \\
& \left.-\eta \sum_{i=1}^{\ell+1}\left(\varphi^{i} \bar{\chi}^{i}-\bar{\varphi}^{i} \chi^{i}\right)-2 \lambda\left(\imath \sigma \sum_{j=1}^{\ell+1}\left|\varphi^{i}\right|^{2}+\sum_{i=1}^{\ell+1} \chi^{i} \bar{\chi}^{i}\right)\right)
\end{aligned}
$$

Define an action of $U_{\ell+1}$ on $\mathbb{C}^{\ell+1}$, so that $j$ th factor in the diagonal subgroup $U_{1}^{\ell+1} \subset U_{\ell+1}$ acts as follows

$$
\mathrm{e}^{\imath \alpha_{j}}: \varphi^{i} \longrightarrow \mathrm{e}^{\imath \alpha_{j} \delta_{i j}} \varphi^{j}
$$

This action is Hamiltonian with respect to (F.1) and the corresponding momenta are given by

$$
\mu_{j}(\varphi)=-\frac{1}{2}\left|\varphi^{j}\right|^{2}
$$

The action of $U_{\ell+1}$ descends to the Hamiltonian action on $\mathbb{P}^{\ell}$ obtained by the Hamiltonian reduction (F.2). Corresponding momenta are given in inhomogeneous coordinates $w_{j}=\varphi_{j} / \varphi_{\ell+1}, \varphi_{\ell+1} \neq 0$ by

$$
\begin{aligned}
\mu_{j}^{\mathbb{P}^{\ell}} & =-\frac{r^{2}}{2} \frac{\left|w_{j}\right|^{2}}{1+\sum_{j=1}^{\ell}\left|w_{j}\right|^{2}}, \quad j=1, \ldots \ell, \\
\mu_{\ell+1}^{\mathbb{P}^{\ell}} & =-\frac{r^{2}}{2} \frac{1}{1+\sum_{j=1}^{\ell}\left|w_{j}\right|^{2}} .
\end{aligned}
$$

Let $c_{1}^{U_{\ell+1}}$ be a $U_{\ell+1}$-equivariant extension of the first Chern class $c_{1}$ of the line bundle $\mathcal{O}(1)$ on $\mathbb{P}^{\ell}$. We would like to express the integral of $\exp \left(a c_{1}^{U_{\ell+1}}\right)$ 
over $\mathbb{P}^{\ell}$ in terms of an integral over larger space generalizing (F.13) to $U_{\ell+1}$-equivariant setting. To simplify the presentation we use the standard properties of equivariant cohomology to replace the calculations in $U_{\ell+1^{-}}$ equivariant cohomology by equivalent calculations in equivariant cohomology with respect to a diagonal subgroup $U_{1}^{\ell+1} \subset U_{\ell+1}$ with additionally imposed condition of an invariance with respect to the Weyl group $W=S_{\ell+1}$ of $U_{\ell+1}$.

In the following, we need a $U_{\ell+1}$-equivariant version of (F.13) which can be obtained straightforwardly generalizing the previous constructions. Thus we just state the main identity in this case. Consider the same set of coordinates as in (F.10) but with the following equivariant de Rham differential $Q$ given by

$$
\begin{array}{r}
Q_{U_{\ell+1}} \varphi^{i}=\chi^{i}, \quad Q_{U_{\ell+1}} \chi^{i}=-\imath\left(\sigma+\sigma_{j}\right) \varphi^{i}, \quad Q_{U_{\ell+1}} \xi=H, \\
Q_{U_{\ell+1}} H=0, \quad Q_{U_{\ell+1}} \lambda=\eta, \quad Q_{U_{\ell+1}} \eta=0,
\end{array}
$$

where $\left(\sigma_{1}, \ldots, \sigma_{\ell+1}\right)$ are elements of the Lie algebra of $U_{1}^{\ell+1}$. The expression for the $U_{1}^{\ell+1}$ equivariant version (F.13) is given by

$$
\begin{aligned}
\int_{\mathbb{P}^{\ell}} & \exp \left(a c_{1}^{U_{1}^{\ell+1}}\right) \\
= & \frac{1}{(2 \pi)^{2}} \int_{\Pi T Y \times \mathbb{R} \times \mathbb{R}^{2 / 2}} \frac{d^{2} \varphi d^{2} \chi d \sigma d \lambda d \xi d \eta d H}{\operatorname{Vol}\left(\mathrm{U}_{1}\right)} \mathrm{e}^{a \sigma} \\
& \times \exp \left(\imath Q_{U_{\ell+1}}\left(\xi\left(-\frac{1}{2} \sum_{j=1}^{\ell+1} \varphi^{j} \bar{\varphi}^{j}+\frac{1}{2} r^{2}\right)+\imath \lambda \sum_{j=1}^{\ell+1}\left(\varphi^{j} \bar{\chi}^{j}-\bar{\varphi}^{j} \chi^{j}\right)\right)\right),
\end{aligned}
$$

where

$$
\begin{aligned}
Q_{U_{\ell+1}} & \left(\xi\left(-\frac{1}{2} \sum_{j=1}^{\ell+1} \varphi^{j} \bar{\varphi}^{j}+\frac{1}{2} r^{2}\right)+\imath \lambda \sum_{j=1}^{\ell+1}\left(\varphi^{j} \bar{\chi}^{j}-\bar{\varphi}^{j} \chi^{j}\right)\right) \\
= & H\left(-\frac{1}{2} \sum_{j=1}^{\ell+1} \varphi^{j} \bar{\varphi}^{j}+\frac{1}{2} r^{2}\right)+\frac{1}{2} \xi \sum_{j=1}^{\ell+1}\left(\varphi^{j} \bar{\chi}^{j}+\bar{\varphi}^{j} \chi^{j}\right) \\
& +\imath \eta \sum_{j=1}^{\ell+1}\left(\varphi^{j} \bar{\chi}^{j}-\bar{\varphi}^{j} \chi^{j}\right)-2 \lambda \sum_{j=1}^{\ell+1}\left(\sigma+\sigma_{j}\right)\left|\varphi^{j}\right|^{2}+2 \imath \lambda \sum_{j=1}^{\ell+1} \chi^{j} \bar{\chi}^{j} .
\end{aligned}
$$


Proposition F.1. Let $\left(\sigma_{1}, \ldots, \sigma_{\ell+1}\right)$ be an element of the Lie algebra of $U_{1}^{\ell+1}$. The $U_{1}^{\ell+1}$-equivariant symplectic volume (F.16) has the following integral representation

$$
\int_{\mathbb{P}^{\ell}} \exp \left(\frac{r^{2}}{2} c_{1}^{U_{1}^{\ell+1}}\right)=(2 \pi)^{\ell-1} \int_{\mathbb{R}-\imath \epsilon} d H e^{\frac{\imath r^{2} H}{2}} \frac{1}{\prod\left(\imath H+\sigma_{j}\right)},
$$

where $\epsilon>\max \left(-\sigma_{j}\right), j=1, \ldots, \ell+1$.

Proof. Integrating over $H, \lambda$ and $\sigma$, we obtain the following:

$$
\begin{aligned}
\left\langle e^{a \sigma}\right\rangle= & \frac{1}{2 \pi} \int \frac{d^{2} \varphi d^{2} \sigma d \xi d \eta}{\operatorname{Vol}\left(\mathrm{U}_{1}\right)} e^{a \sigma(\varphi, \chi)} \delta\left(-\frac{1}{2} \sum_{j=1}^{\ell+1}\left|\varphi_{j}\right|^{2}+\frac{1}{2} r^{2}\right) \frac{1}{2 r^{2}} \\
& \times \exp \left\{\left(\frac{\imath \xi}{2}-\eta\right) \sum_{j=1}^{\ell+1} \varphi^{j} \bar{\chi}^{j}+\left(\frac{\imath \xi}{2}+\eta\right) \sum_{j=1}^{\ell+1} \bar{\varphi}^{j} \chi^{j}\right\}
\end{aligned}
$$

where

$$
\sigma(\chi, \varphi)=\frac{1}{r^{2}}\left(\imath \sum_{j=1}^{\ell+1} \chi^{j} \bar{\chi}^{j}-\sum_{j=1}^{\ell+1} \sigma_{j}\left|\varphi_{j}\right|^{2}\right)
$$

Here, we use the following normalization of the integration measure:

$$
d^{2} \varphi d^{2} \chi=\prod_{j=1}^{\ell+1} \frac{\imath}{2} d \varphi^{j} d \bar{\varphi}^{j} \prod_{j=1}^{\ell+1} \frac{2}{\imath} d \chi^{j} d \bar{\chi}^{j}
$$

It is useful to reintroduce the variable $H$ by writing the first delta-function in (F.18) in the integral form. Then integrating over odd variables $d \xi d \eta$ and $d^{2} \chi$ and taking into account that $\operatorname{Vol}\left(U_{1}\right)=2 \pi$ we arrive at

$$
\left\langle\mathrm{e}^{a \sigma}\right\rangle=\frac{1}{(2 \pi)^{2}}\left(\frac{2 a}{r^{2}}\right)^{\ell} \int d H \int \prod_{j=1}^{\ell+1} \frac{\imath}{2} d \varphi^{j} d \bar{\varphi}^{j} \mathrm{e}^{+\frac{\imath r^{2}}{2} H} \mathrm{e}^{-\frac{1}{2} \sum \varphi^{j}\left(\imath H+\frac{2 a}{r^{2}} \sigma_{j}\right) \bar{\varphi}^{j}}
$$

For a finite-dimensional Gaussian integral, we have

$$
\int_{\mathbb{C}^{N}} \mathrm{e}^{-\frac{1}{2} \sum_{i, j=1}^{N} \bar{z}_{i} A_{i j} z_{j}} \prod_{j=1}^{N} \frac{\imath}{2} d z^{j} d \bar{z}^{j}=\frac{1}{\operatorname{det} A / 2 \pi}
$$


where the matrix $A$ has positive eigenvalues. More generally, the Gaussian integral (F.20) for $A$ having complex eigenvalues $a_{j}$ such that $\operatorname{Re}\left(a_{j}\right) \geq$ $0, j=1, \ldots, N$ is defined as a limit of the integral for $A$ having complex eigenvalues $a_{j}$ such that $\operatorname{Re}\left(a_{j}\right)>0, j=1, \ldots, N$ expressed through (F.20). Now assuming $\operatorname{Re}\left(\imath H+\frac{2 a}{r^{2}} \sigma_{j}\right)>0, j=1, \ldots, \ell+1$ and taking integral over $\varphi$, we obtain

$$
\left\langle\mathrm{e}^{a \phi}\right\rangle=\left(\frac{2 a}{r^{2}}\right)^{\ell}(2 \pi)^{\ell-1} \int_{\mathbb{R}-\imath \epsilon} d H e^{\frac{\imath r^{2} H}{2}} \frac{1}{\prod\left(\imath H+\frac{2 a}{r^{2}} \sigma_{j}\right)},
$$

where $\epsilon>\max \left(-\frac{2 a}{r^{2}} \sigma_{j}\right), j=1, \ldots, \ell+1$. Taking $a=\frac{r^{2}}{2}$, we finally obtain

$$
\left\langle\mathrm{e}^{\frac{r^{2}}{2} \phi}\right\rangle=(2 \pi)^{\ell-1} \int_{\mathbb{R}-\imath \epsilon} d H \mathrm{e}^{\frac{\imath^{2} H}{2}} \frac{1}{\prod\left(\imath H+\sigma_{j}\right)},
$$

where $\epsilon>\max \left(-\sigma_{j}\right), j=1, \ldots, \ell+1$. This completes the proof of the proposition.

Finally, let us provide a reformulation of the integral representation of the integrals of the equivariantly closed forms over $\mathbb{P}^{\ell}$ that does not include integrations over odd variables.

Lemma F.1. Let $\sigma_{j}>0, j=1, \ldots, \ell+1$. The following identity holds:

$$
\frac{1}{2 \pi} \int_{\mathbb{C}^{\ell+1}} \delta\left(\mu_{U(1)}+r^{2} / 2\right) \mathrm{e}^{\left(\omega_{\mathbb{C}^{\ell+1}}+\sum_{j=1}^{\ell+1} \sigma_{j} \mu_{j}\right)}=\int_{\mathbb{P}^{\ell}} \mathrm{e}^{\left(\omega_{\mathbb{P} \ell}+\sum_{j=1}^{\ell+1} \sigma_{j} \mu_{j}^{\mathrm{P}}\right)},
$$

where $\omega_{\mathbb{P}^{\ell}}$ is given by (F.3) and the reduced Hamiltonians $\mu_{j}^{\mathbb{P}^{\ell}}$ are given by (F.14) and (F.15).

Proof. This can be deduced from the previous considerations but allows simple direct derivation. Let us introduce new variables $w_{j}=\varphi_{j} \varphi_{\ell+1}$, 
$j=1, \ldots, \ell$ and $t=\left|\varphi_{\ell+1}\right|^{2}, \theta=\frac{1}{2 \imath} \ln \frac{\varphi_{\ell+1}}{\bar{\varphi}_{\ell+1}}$, so that $\varphi_{\ell+1}=\sqrt{t} \mathrm{e}^{\imath \theta}$. Then we have

$$
\begin{aligned}
& \frac{1}{2 \pi}\left(\frac{\imath}{2}\right)^{\ell+1} \int_{\mathbb{C}^{\ell+1}} \bigwedge_{i=1}^{\ell+1} d \varphi_{i} \wedge d \bar{\varphi}_{i} \delta\left(\frac{1}{2} \sum_{i=1}^{\ell+1}\left|\varphi_{i}\right|^{2}-\frac{r^{2}}{2}\right) \mathrm{e}^{\sum_{j=1}^{\ell+1} \sigma_{j} \mu_{j}} \\
&= \frac{r^{2 \ell}}{2 \pi}\left(\frac{\imath}{2}\right)^{\ell} \int_{0}^{2 \pi} d \theta \int_{0}^{\infty} d t t^{\ell} \int_{\mathbb{C}^{\ell}} \frac{\bigwedge_{n=1}^{\ell}\left(d w_{n} \wedge d \bar{w}_{n}\right)}{1+\sum\left|w_{n}\right|^{2}} \\
& \times \delta\left(t-\frac{r^{2}}{1+\sum\left|w_{n}\right|^{2}}\right) \mathrm{e}^{\sum_{j=1}^{\ell+1} \sigma_{j} \mu_{j}} \\
&= r^{2 \ell}\left(\frac{\imath}{2}\right)^{\ell} \int_{\mathbb{C}^{\ell}} \frac{\bigwedge_{n=1}^{\ell}\left(d w_{n} \wedge d \bar{w}_{n}\right)}{\left(1+\sum\left|w_{n}\right|^{2}\right)^{\ell+1}} \mathrm{e}^{\sum_{j=1}^{\ell+1} \sigma_{j} \mu_{j}^{\mathbb{p}^{\ell}}} .
\end{aligned}
$$

Taking into account that

$$
\frac{\omega_{\mathbb{P} \ell}^{\ell}}{\ell !}=r^{2 \ell}\left(\frac{\imath}{2}\right)^{\ell} \frac{\bigwedge_{n=1}^{\ell}\left(d w_{n} \wedge d \bar{w}_{n}\right)}{\left(1+\sum\left|w_{n}\right|^{2}\right)^{\ell+1}},
$$

we obtain the identity (F.23).

Corollary F.1. Let $\mathbb{C}^{\ell+1}=\mathbb{C}^{n_{1}} \oplus \cdots \oplus \mathbb{C}^{n_{k}}, \sum_{a=1}^{k} \operatorname{dim}\left(V_{a}\right)=\ell+1$ be $a$ decomposition of the symplectic space vector space $\left(\mathbb{C}^{\ell+1}, \Omega\right)$, where symplectic structure is given by (F.1) and $\left(\varphi_{n_{1}+\cdots n_{a-1}+1}, \ldots, \varphi_{n_{1}+\cdots n_{a}}\right)$ are coordinates on $\mathbb{C}^{n_{a}}$. Let $U_{1}^{\ell+1}$ act on $\mathbb{C}^{\ell+1}$ diagonally. The action is Hamiltonian and let $\mu_{j}, j=1, \ldots \ell+1$ be the momenta corresponding to the action of $j$-th factor $\left(U_{1}\right)_{j}$. Let $U_{1}^{k} \subset U_{1}^{\ell+1}$ act on $\mathbb{C}^{\ell+1}$ so that the a-th $\left(U_{1}\right)_{a}$ acts non-trivially only on $\mathbb{C}^{n_{a}}$ by multiplication on complex numbers. Let $U_{1}$ be diagonally embedded in $U_{1}^{k}$. Let $\mathbb{P}\left(\mathbb{C}^{\ell+1}\right)$ be a Hamiltonian reduction of $\mathbb{C}^{\ell+1}$ over $U_{1}$ with momentum $x$ and $\mathbb{P}\left(\mathbb{C}^{n_{a}}\right)$ be a Hamiltonian reduction of $\mathbb{C}^{n_{a}}$ over $\left(U_{1}\right)_{a}$ with the momentum $x_{a}$. Then the following relation between $U_{k}$-equivariant integrals holds

$$
\begin{aligned}
& \int_{\mathbb{P}\left(\mathbb{C}^{\ell+1}\right)} \mathrm{e}^{\omega_{\mathbb{P}\left(\mathbb{C}^{\ell+1}\right)}(x)+\sum_{j=1}^{\ell+1} \sigma_{j} \mu_{j}^{\mathbb{P}\left(\mathbb{C}^{\ell+1}\right)}} \\
& =\frac{1}{2 \pi} \int d x_{1} \cdots d x_{k} \delta\left(x-\sum_{j=1}^{k} x_{j}\right) \\
& \times \prod_{a=1}^{k} 2 \pi \int_{\mathbb{P}\left(\mathbb{C}^{n_{a}}\right)} \mathrm{e}^{x_{a} \omega_{\mathbb{P}\left(\mathbb{C}^{n}\right)}+\sum_{i=n_{1}+\ldots n_{a-1}+1}^{n_{1}+\ldots+n_{a}} \sigma_{i} \mu_{i}^{\mathbb{P}\left(\mathbb{C}^{n_{a}}\right)}},
\end{aligned}
$$


where $\omega_{\mathbb{P}\left(\mathbb{C}^{n_{a}}\right)}$ is a standard Fubini-Studi symplectic form on $\mathbb{P}\left(\mathbb{C}^{n_{a}}\right)$ multiplied by $x_{j}$ and $\mu_{j}^{\mathbb{P}\left(\mathbb{C}^{n a}\right)}$ is a momentum for the action of $\left(U_{1}\right)_{j}$.

Proof. This identity straightforwardly follows from Lemma F.1 and the following identity:

$$
\int d x_{1} \cdots d x_{k} \prod_{j=1}^{k} \delta\left(\mu_{j}+x_{j}\right) \delta\left(x-\sum_{j=1}^{k} x_{j}\right)=\delta\left(\sum_{j=1}^{k} \mu_{k}+x\right) .
$$

\section{References}

[1] J. Adams, D. Barbash and D. A. Vogan Jr., The Langlands classification and irreducible characters of real reductive groups, Progress in Mathematics, 104, Birkhäuser Boston Inc., Boston, MA, 1992.

[2] A. Astashkevich and V. Sadov, Quantum cohomology of partial flag manifolds $F_{n_{1}, \ldots, n_{k}}$, Commun. Math. Phys. 170 (1995), 503-528.

[3] M. Audin, Torus actions on symplectic manifolds, Progress in Mathematics, Birkhäuser Boston, Inc., Boston, MA, 2004.

[4] V. Batyrev, I. Ciocan-Fontanine, B. Kim and D. van Straten, Mirror symmetry and toric degenerations of flag manifolds, [arXiv:math.AG/9803108].

[5] A. Beilinson and V. Drinfeld, Quantization of Hitchins integrable system and Hecke eigen-sheaves, preprint 1995, http://www.math.uchicago.edu/arinkin/langlands/.

[6] J. Bernstein and S. Gelbart (eds.), An introduction to the Langlands program, Lectures presented at the Hebrew University of Jerusalem, Jerusalem, March 12-16, 2001, Birkhäuser Boston, Inc., Boston, MA, 2003.

[7] D. Bump, Automorphic forms and representations, Cambridge University Press, Cambridge, 1998.

[8] W. Casselman and J. Shalika, The unramified principal series of p-adic groups II. The Whittaker function. Compositio Math. 41 (1980) 207231. 
[9] I. Ciocan-Fontanine, On quantum cohomology rings of partial flag varieties, Duke Math. J. 98 (1999), 485-524.

[10] S. Cordes, G. Moore and S. Ramgoolam, Lectures on 2D Yang-Mills theory, equivariant cohomology and topological field theories, In Proceedings of the 1994 Les Houches School on Fluctuating Geometries, Nucl. Phys. Proc. Suppl. 41 (1995) 184-244. [arXiv:hep-th/9411210].

[11] P. Deligne, P. Etingof, D. Freed, L. Jeffrey, D. Kazhdan, J. Morgan, D. Morrison and E. Witten (eds), Quantum fields and strings: a course for mathematicians, American Mathematical Society, Providence, RI, 1999.

[12] J. J. Duistermaat and G. J. Heckman, On the variation in the cohomology of the symplectic form of the reduced phase space, Invent. Math. 69(2) (1982), 259-268.

[13] A. Gerasimov, S. Kharchev and D. Lebedev, Representation theory and quantum inverse scattering method: the open Toda chain and the hyperbilic Sutherland model, Int. Math. Res. Not., 17 (2004), 823-854.

[14] A. Gerasimov, S. Kharchev, D. Lebedev and S. Oblezin, On a GaussGivental representation of quantum Toda chain wave function, Int. Math. Res. Notices (2006), Aricle ID96489, 23 p.

[15] A. Gerasimov, D. Lebedev and S. Oblezin, New integral representations of Whittaker functions for classical groups, [arXiv:math.RT/0705.2886].

[16] A. Gerasimov, D. Lebedev and S. Oblezin, On q-deformed $\mathfrak{g l}_{\ell+1^{-}}$ Whittaker functions I,II,III, Commun. Math. Phys. 294 (2010), 97-119, [arXiv:0803.0145]; Commun. Math. Phys. 294 (2010), 121-143, [arXiv:0803.0970]; Lett. Math. Phys. 97 (2011), 1-24, [arXiv:0805.3754].

[17] A. Gerasimov, D. Lebedev and S. Oblezin, Archimedean L-factors and topological field theories I, Commun. in Number Theory and Physics, 5 (1) 2011, [arXiv:0906.1065].

[18] A. Gerasimov, D. Lebedev and S. Oblezin, Archimedean L-factors and topological field theories II, Commun. in Number Theory and Physics, 5 (1) 2011; [arXiv:0909.2016].

[19] A. Givental, Equivariant gromov - Witten invariants, Int. Math. Res. Not. 1996, 13, 613-663, [arXiv:alg-geom/9603021]. 
[20] A. Givental, Homological geometry I. Projective hypersurfaces, Sel. Math., N.S. 1, 2 (1995), 325-345.

[21] A. Givental, Stationary phase integrals, quantum Toda lattices, flag manifolds and the mirror conjecture, in 'Topics in Singularity Theory', Amer. Math. Soc. Transl. Ser. 2, 180, AMS, Providence, Rhode Island, 1997, pp. 103-115, [arXiv:alg-geom/9612001].

[22] A. Givental and Y.-P. Lee, Quantum K-theory on ag manifolds, nitedierence Toda lattices and quantum groups, Invent. Math. 151 (2003), 193-219, [arXiv:math.AG/0108105].

[23] R. A. Gustafson, Some q-beta and Mellin-Barnes integrals on compact Lie groups and Lie algebras. Trans. Amer. Math. Soc. 341(1) (1994), 69-119.

[24] K. Hori and C. Vafa, Mirror symmetry, [arXiv:hep-th/0002222].

[25] T. Hausel and M. Thaddeus, Mirror symmetry, Langlands duality, and the Hitchin system, [arXiv:math.AG/0205236].

[26] A. Kapustin and E. Witten, Electric-magnetic duality and the geometric Landlands program, Commun. Number Theory Phys. 1 (2007), 1-236, [arXiv:hep-th/0604151].

[27] B. Kim, Quantum cohomology of partial flag manifolds and a residue formula for their intersection pairings, Int. Math. Res. Not. 1 (1995), 1-16.

[28] Y. Manin and A. Panchishkin, Introduction to modern number theory, 2 edn., Springer, 2005.

[29] D. Morrison and R. Plesser, Summing the instantons: quantum cohomology and mirror symmetry in toric varieties, Nucl. Phys. B440 (1995), 279-354, [arXiv:hep-th/9412236].

[30] M. Semenov-Tian-Shansky, Quantization of open Toda lattice, in Encyclopedia of Mathematics Sciences, 16, Springer Verlag, 1994, 226-259.

[31] T. Shintani, On an explicit formula for class 1 Whittaker functions on $G L_{n}$ over p-adic fields. Proc. Japan Acad. 52 (1976), 180-182.

[32] A. Voros, Spectral functions, special functions and the Selberg Zeta function, Commun. Math. Phys. 110 (1987), 439-465. 
[33] E. Witten, Mirror manifolds and topological field theory, in Essays on mirror manifolds, pp. 120-158, Internat. Press, Hong Kong, 1992, [arXiv:hep-th/9112056].

[34] E. Witten, Phases of $N=2$ theories in two dimensions, Nucl. Phys. B 403 (1993), 159-222, [arXiv:hep-th/9301042].

[35] E. Witten, The Verlinde algebra and the cohomology of the Grassmannian, in 'Geometry, Topology, and Physics' (Cambridge, Mass., 1993), Conf. Proc. Lecture Notes Geom. Topology 4, Internat. Press, Cambridge, Mass., 1995, 357-422, [arXiv:hep-th/9312014].

Institute for Theoretical and Experimental Physics

117259, Moscow

Russia

SCHOOL OF Mathematics

Trinity College Dublin

DUBLIN 2

IRELAND

Hamilton Mathematics Institute

Trinity College Dublin,

DUBLIN 2

IRELAND

ReCEIVED July 29, 2010 
